Portland State University

PDXScholar

$1-1-2010$

\title{
Genomic and Hormonal Components of Altered Developmental Pathways in the Annual Killifish, Austrofundulus limnaeus
}

Benjamin M. Pri-Tal

Portland State University

Follow this and additional works at: https://pdxscholar.library.pdx.edu/open_access_etds Let us know how access to this document benefits you.

Recommended Citation

Pri-Tal, Benjamin M., "Genomic and Hormonal Components of Altered Developmental Pathways in the Annual Killifish, Austrofundulus limnaeus" (2010). Dissertations and Theses. Paper 167.

https://doi.org/10.15760/etd.167

This Thesis is brought to you for free and open access. It has been accepted for inclusion in Dissertations and Theses by an authorized administrator of PDXScholar. Please contact us if we can make this document more accessible: pdxscholar@pdx.edu. 
Genomic and Hormonal Components of Altered Developmental Pathways in the Annual Killifish, Austrofundulus limnaeus

by

Benjamin M. Pri-Tal

a thesis submitted in partial fulfillment of the requirements for the degree of

\author{
Master of Science \\ in \\ Biology
}

Thesis Committee:

Jason E. Podrabsky, chair

Stanley S. Hillman

Todd N. Rosenstiel

Portland State University

2010 


\begin{abstract}
The annual killifish, Austrofundulus limnaeus, may enter embryonic diapause at three distinct points of development, termed diapause I, II, and III. Previous studies suggest a role for steroid hormones in the regulation of diapause in annual killifish. This study concerns the hormonal and genomic components involved in the developmental decision to enter or escape diapause II from both a maternal and embryonic perspective. Steroid hormone levels were measured in tissues isolated from adult female fish that were producing either high or low proportions of escape embryos. Levels of steroid hormones were also measured in new fertilized embryos that were known to be on either an escape or diapausing developmental trajectory. In addition, cDNA microarray gene expression analysis was used to identify gene sequences that may be associated with the regulation of entry into diapause in this species. Decreases in maternal estrogen levels associated with aging are correlated with decreasing escape embryo production, but there is no direct association between measured steroid hormone levels and escape embryo production. However, maternal production of escape embryos is correlated with increased ratios of $17 \beta$-estradiol to testosterone in ovary tissue, and cDNA microarray gene expression analysis indicates differentially regulated sequences associated with escape embryo production in maternal tissues. Both of these independent measures suggest hormonal involvement in the regulation of diapause. Embryonic levels of steroid hormones in newly fertilized embryos are not correlated with entry or escape from diapause II, although
\end{abstract}


incubation in exogenous cortisol and $17 \beta$-estradiol causes an increase in the proportion of escape embryos. Gene expression analysis again suggests hormonal involvement. Interestingly, genes involved in epigenetic control of gene expression though chromatin condensation are differentially regulated in both maternal tissues producing escape embryos, and in embryos on the different developmental trajectories. These data suggest that hormonal control of gene expression through alterations of chromatin condensation may regulate the decision to enter or escape diapause II. 


\section{Table of contents}

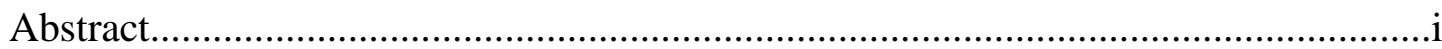

List of

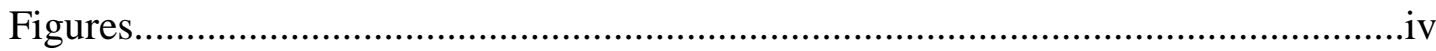

List of

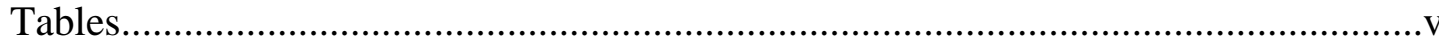

Chapter 1

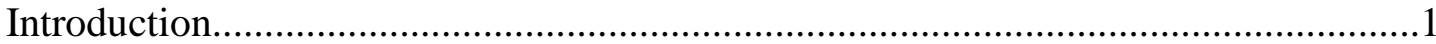

Chapter 2

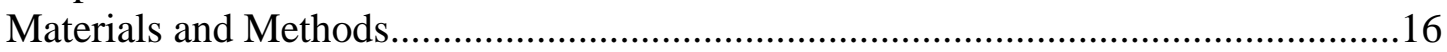

Chapter 3

Results.

Chapter 4

Discussion.

References

Appendix A

Table 1 sequences.

Appendix B

Table 2 sequences

Appendix C

Table 3 sequences.

102

Appendix D

Table 4 sequences

Appendix E

Table 5 sequences 


\section{List of figures}

Figure 1. Levels of $17 \beta$-estradiol in (A) ovarian tissue and (B) plasma of female Austrofundulus limnaeus as a function of age.

Figure 2. Hormone levels in ovarian tissues isolated from young female A. limnaeus

Figure 3. The relationship between the ratio of $17 \beta$-estradiol to (A) testosterone, and $(\mathrm{B})$ progesterone as a function of lifetime escape embryo production in young females

Figure 4. Hormone concentrations in newly fertilized embryos presented (A) per embryo and (B) per gram embryo tissue.

Figure $5.17 \beta$-estradiol (A) and Androstendione (B) levels through early development.

Figure 6. Effect of incubation in exogenous (A) $17 \beta$-estradiol and (B) cortisol on the proportion of embryos that escape diapause II.

Figure 7. Hormone levels through embryonic $17 \beta$-estradiol treatment.

Figure 8. Hormone levels in $17 \beta$-estradiol treated and control dechorionated embryos

Figure 9. The distribution of sequences found to be differentially expressed in escape embryos and tissues isolated from females producing escape embryos.

Figure 10 . The number of differentially regulated genes identified in each microarray experiment and the distribution of shared sequences. 


\section{List of tables}

Table 1. Differentially regulated genes in newly fertilized embryos (upregulated).

Table 2. Differentially regulated genes in newly fertilized embryos (downregulated).

Table 3. Differentially regulated genes in 14-16 somite embryos.

Table 4. Differentially regulated genes in ovary of adult fish.

Table 5. Differentially regulated genes in liver of adult fish. 


\section{Introduction}

The uniting factor of most environments is variability. Even in the most stable environments, such as Antarctic waters that are constantly at the freezing point of sea water, seasonal changes in food availability and photoperiod may perturb an organism's function. Often, environmental variation challenges an animal from a metabolic, thermal, or hydration perspective. In an aquatic environment, a lack of water availability typically leads to death of the organism and can lead to local population extinctions. Vertebrates have developed different strategies for coping with extremes in environmental variation; some develop tolerance or adapt to thrive in these conditions, while others avoid periods of unfavorable environmental conditions by migration or by entering a state of metabolic dormancy. Those organisms that use dormancy typically exhibit strategies that consist of lowering metabolic and physiological set points to reduce energy consumption and thus prolong survival in the absence of active resource acquisition. Diapausing embryos of the annual killifish are one of the most extreme examples of metabolic dormancy among vertebrates. However, very little is known about the physiological or biochemical underpinnings that control entry into dormancy in this species. The focus of this thesis is to explore these mechanisms.

Metabolic depression has long fascinated physiologists; Anton van Leeuwenhoek noted the dormant state of rotifers found in the gutters of house roofs over 300 years ago (in Guppy, 2004). Dormancy takes many forms in vertebrates, and 
is mobilized in reaction to different environmental challenges. In the extreme seasonality of latitudes approaching the poles, animals may enter hibernation for the cold season, sleeping away the bitter cold in a den or burrow. For example, when winter arrives the Ranid frog, Rana sylvatica, undergoes biochemical changes that allow for the survival of freezing temperatures. At more tropical latitudes, escape from heat or lack of water through estivation is a common strategy, such as the Malagasy fat-tailed dwarf lemurs that rest away the hot season in dens (Dausmann et. al., 2004). The African lungfish covers itself in a desiccation resistant cocoon to rest through the dry periods of its ephemeral aquatic habitat (Janssens, 1965).

The unifying factor in all of these different strategies of avoiding unfavorable conditions is depressed metabolism; to quote the comparative physiologist Kjell Johanssen, these animals are capable of "turning down to the pilot light" (in Hochachka et. al, 1997). Through metabolic depression animals "wait out" periods of extreme environmental challenge and escape from "biological time", decreasing their needs for water, oxygen, or heat (Hochachka and Somero, 2002). Indeed, this is an effective strategy as states of developmental arrest are found in species of all major animal phyla (Hand, 1991; Yuan et al., 2007).

Environmental physiology is the study of how animals manifest different responses to their environment, from the molecular and cellular level to that of the entire organism. These responses may be realized across various time scales ranging from evolutionary time to almost immediate adjustments. On the more immediate time scales (less than the lifetime of an organism) the interaction between genotype 
and its external manifestation under different conditions (phenotype) can be adjusted in response to environmental variation. Phenotypic plasticity allows an individual to manifest varied responses to different environmental conditions. In many situations, developmental arrest in diapause is manifested as a form of phenotypic plasticity (Marcus, 1979; Denlinger, 1979; Caceres, 1998; Hairston et al., 1996; Drinkwater and Crowe, 2005; Gerisch and Antebi, 2004). This is also the case for embryos of Austrofundulus limnaeus that have recently been shown to exhibit plasticity in developmental pathways controlled by both environmental and maternal cues (Podrabsky et al., 2010). The unique life history and the amount of scientific information available on A. limnaeus make it an ideal candidate for studies on the factors involved in, and influencing, embryonic diapause.

\section{The Biology of Annual Killifish}

Annual killifish persist in an inhospitable environment, eking out a precarious existence where few other fish could survive. Annual killifish live in savanna and desert regions in Africa and South America that experience great seasonal variation in rainfall (Wourms, 1972a). Ephemeral ponds inhabited by the South American annual killifish, Austrofundulus limnaeus, exhibit large diurnal fluctuations in temperature, dissolved oxygen content, and pH values (Podrabsky et. al., 1998). Upon desiccation of the pools, adult and juvenile fish perish (Myers, 1952). Populations in a given location persist through the dry season as diapausing embryos buried in the soil. When rains return and pools refill, fish hatch and repopulate the new aquatic habitat. 
Annual killifish are named as such due to their short life spans; typically around a year or less. In the laboratory, A. limnaeus become senescent at approximately one year of age with a maximum life span of about 1.5 years (Podrabsky, 1999). This is consistent with selection for quick growth and attainment of sexual maturity with a tradeoff for longevity, as would be expected in an animal from such a harsh and temporally limited environment. A species of African annual killifish, Nothobranchius furzeri, has one of the shortest life spans reported, with animals in the laboratory living less than 12 weeks (Valdesalici and Cellerino, 2003), and wild type a maximum of 25-32 weeks (Terzibasi et al., 2008). However, the study reporting the shorter lifespan was performed on extremely inbred laboratory fish, as determined by homozygosity of typically polymorphic loci (Terzibasi et al., 2008). The extremely short lifespan of these fish has made them a proposed model in vertebrate aging research, as well as in studies of experimental evolution and the effect of lifelong pharmaceutical treatments (reviewed in Genade et el., 2005). Vertebrates exhibiting such naturally short life spans can be extremely useful as a model for other more long-lived vertebrate species, that are significantly more difficult to study in a multigenerational context due to the constraints of typical human time frames.

\section{Diapause in Annual Killifish}

Unique among vertebrates, annual killifish exhibit three distinct developmental stages in which embryonic development may be arrested; these stages 
of diapause have been termed diapause I, II, and III (Wourms, 1972a). Each of these three stages confers increased resistance to environmental insult. In various combinations, the three different stages of developmental arrest can produce eight different developmental trajectories. Ecologically, this staggering of developmental progression likely provides security in a varied environment where early or untimely emergence could otherwise lead to population extinction (Wourms, 1972c).

Diapause I is a developmental arrest interjected between epiboly and embryogenesis, a time that is typically associated with gastrulation in most species of fish. During epiboly, blastomeres form two distinct populations; deep blastomeres that consolidate as a mass and then migrate outwards as amoeboid cells, and outer blastomeres that form the enveloping cell layer. Epiboly commences in annual killifish with a low number of blastomeres (200-1000) compared to other teleosts such as Fundulus that begins epiboly with around 27,500 blastomeres (Wourms, 1972b). Diapause I occurs following dispersion of the embryonic blastomeres shortly after the completion of epiboly, but prior to the reaggregation of cells that constitute the embryonic axis. In Austrofundulus, arrest at diapause I is facultative and can be of variable duration, being composed of developmental arrest between stages 20 and 21 (Wourms, 1972c). A comparative study by Wourms (1972b) including 43 species of both annual and non-annual killifish, found that dispersion and reaggregation of the blastomeres and diapause I are only common to the killifish exhibiting an annual life cycle (i.e. dispersion and reaggregation are missing from species that do not exhibit diapause). Three benefits to the dispersion-reaggregation developmental 
pattern have been hypothesized: (1) a period of developmental arrest can be interjected without disruption of neurulation, (2) the enveloping cell layer may become specialized to confer resistance to desiccation and osmotic stress, and (3) dispersed cells (if totipotent) can be sacrificed without affecting overall embryo survival (Wourms, 1972b).

Arrest at diapause II has been the most extensively studied of the embryonic diapause stages in annual killifish. The physiology of anoxia tolerance (Duerr and Podrabsky, 2010; Podrabsky et. al., 2007; Fergusson-Kolmes and Podrabsky, 2007), desiccation tolerance (Podrabsky et al., 2001), salinity tolerance (Machado and Podrabsky, 2007), bioenergetics (Duerr and Podrabsky, 2010; Podrabsky and Hand, 1999), protein synthesis (Podrabsky and Hand, 2000), and induction of molecular chaperones (Podrabsky and Somero, 2007), have all been studied in A. limnaeus entering diapause II. This stage is initiated at approximately 24 days post fertilization (dpf), in the long-somite embryo stage (Wourms, 1972a; Podrabsky and Hand, 1999). At this diapause stage, embryos have 38-40 pairs of somites, a beating heart, optic cups, and other basic elements of the central nervous system (Podrabsky and Hand, 1999). Diapause II embryos exhibit a depression of up to $90 \%$ of the pre-diapause oxygen consumption and heart rate. Both metabolism and heart rate increase during early development, and then begin to decline several days prior to cessation of development in diapause II (Podrabsky and Hand, 1999). Thus, it appears that the molecular events that signal for entry into diapause likely occur many days prior to 
the cessation of morphological development associated with diapause II. This is an important point to consider when searching for the signals that induce diapause II.

A small proportion of the embryos from a given spawning event will not enter diapause II but instead develop through an alternate pathway (Podrabsky et al., 2010). Wourms (1972c) named these "escape embryos" because they escape from arrest of development in diapause II. Escape embryos can be distinguished morphologically from diapausing embryos when they reach stages that possess about 18-20 pairs of somites. Escape embryos exhibit a number of developmental characteristics that do not form until several days after diapause II breakage in arresting embryos. For example, escape embryos are covered on the dorsal aspect of the head and trunk with melanocyte cells, hemoglobin is expressed in circulating red blood cells, the vasculature of the yolk sac is greatly increased, chondrocyte condensations (otolith primordia) can be observed in the developing otic vesicles, and the early vestiges of the gut have formed. Escape embryos possess lower anaerobic and aerobic capacity at the termination of development than do embryos that have entered diapause II (Chennault and Podrabsky, 2010). This may indicate a tradeoff in development, where the rapidly developing escape embryos reach hatching at faster rates, but with lower metabolic capabilities when compared to embryos that have arrested development at diapause II (Chennault and Podrabsky, 2010).

Diapause III is a state of arrested development that occurs directly prior to hatching, when the embryos are fully developed. Austrofundulus embryos entering diapause III may remain dormant for over 100 days (Wourms, 1972c). This 
developmental arrest is thought to be obligate in most embryos of A. limnaeus, although small subpopulations $(\approx 10 \%)$ bypass developmental arrest at diapause III and hatch immediately when the embryos are reared in a laboratory environment (Wourms, 1972c). Like diapause II, diapause III is also characterized by significant metabolic depression (Podrabsky and Hand, 1999). Interestingly, while metabolism decreases during this stage of developmental arrest, anaerobic and aerobic capacities increase (Chennault and Podrabsky, 2010). This may indicate different control mechanisms compared to diapause II. Diapause III may be rapidly terminated, and the immediate capacity to reverse metabolic arrest is likely necessary for posthatching success (Chennault and Podrabsky, 2010). 


\section{Environmental Tolerance During Diapause}

Anoxia tolerance in embryos of A. limnaeus is substantial during early development, and increases to the highest levels during diapause II. At 4 dpf, $\mathrm{LT}_{50}$ values for anoxia are near 20 days. At $32 \mathrm{dpf}$, about a week after the induction of diapause II, these values reach over 65 days. This anoxia tolerance continues through 4 days post-diapause II (dpd), but is largely lost by $8 \mathrm{dpd}$. Anaerobic metabolic capacity is high throughout development in embryos of A. limnaeus, as evidenced by high lactate dehydrogenase (LDH) to citrate synthase (CS) ratios (Chennault and Podrabsky, 2010). At all examined stages of development, embryos entered anoxia induced quiescence, demonstrated by the cessation of morphological development, and physiological activity (Podrabsky et. al., 2007). During this anoxia induced quiescence, embryos in early developmental stages (diapause II and 4 dpd) may cease heart contractility, while later embryonic stages suffer severe bradycardia (FergussonKolmes and Podrabsky, 2007). Early embryos demonstrate higher resistance and recovery rates from anoxic conditions than do later embryos, suggesting the cessation of heart contractility plays a role in tolerance of anoxia (Fergusson-Kolmes and Podrabsky, 2007). Embryos in diapause II show extremely low levels of aerobic capacity, as measured by CS enzymatic activity. This may indicate active suppression of mitochondrial metabolism in embryos arresting development at this stage (Chennault and Podrabsky, 2010).

The ability to resist dehydrating conditions is one of the most pronounced adaptations that permit the unique lifestyle of annual killifish. Annual killifish may 
spend the majority of their lives in embryonic diapause under desiccated conditions (Wourms, 1972c). This tolerance to dehydration reaches its highest levels during diapause II (Podrabsky et al., 2001). A. limnaeus survive dehydrating conditions by reducing evaporative water loss to undetectable levels after 32 days of exposure to dehydrating conditions. Embryos show an initial loss of water from the perivitelline space (around $50 \%$ of the total embryo water), and also an increase in $\beta$-sheet contacts in the egg envelope with dehydration. These embryos show unprecedented desiccation tolerance for an aquatic vertebrate, with greater than $40 \%$ survival after 113 days at $75.5 \%$ relative humidity (Podrabsky et. al., 2001).

Consistent with the overall depression of metabolic and physiological turnover, protein synthesis is decreased in embryos during diapause II (Podrabsky and Hand, 1999). Prior to diapause, $36 \%$ of ATP utilization is diverted to protein synthesis; this approaches zero during diapause II (Podrabsky and Hand, 2000). The decreased protein synthesis during diapause II suggests that proteins conferring the environmental tolerance of embryos may be produced during the metabolically active period leading up to the cessation of growth. In support of this theory, an inducible 70 $\mathrm{kDa}$-class heat shock protein is constitutively expressed during early development and at the highest levels in diapause II embryos (Podrabsky and Somero, 2007). Heat shock proteins can act as molecular chaperones, aiding proteins in retaining proper structure under environmentally challenging regimens. The induction of heat shock proteins was found to be integral to environmental stress tolerance in the diapause of the fleshfly Sarcophaga crassipalpis (Rinehart et al., 2007), the moth Sesamia 
nonagrioides (Gkouvitsas et al., 2009), and the brine shrimp Artemia fransicana (Willsie and Clegg, 2001), suggesting the initiation of environmentally protective gene products may be a common factor in developmental arrest.

\section{Regulation of Diapause Induction}

Substantial variation exists in the relative ratios of diapause to escape embryos produced, both between females under constant conditions, and throughout an individual's life (Podrabsky et al., 2010). Despite a population-level pattern for the reduced production of escape embryos as a female ages, some females may exclusively produce either escape or diapausing embryos, while others produce a mixture of the two types (Podrabsky et al., 2010). In addition, embryos incubated under identical conditions following fertilization consistently show different developmental strategies. Thus, there appears to be significant maternal control over induction of diapause II. The mode of maternal transfer is currently unknown, but may include the packaging of proteins or mRNAs into embryos that control entry into diapause II.

The specific signaling molecules governing the induction of embryonic diapause in annual killifish are unknown. However, some evidence suggests a waterborne factor produced by both male and female adult fish inhibits development at diapause I and II in the East African annual killifish Nothobranchius guentheri (Inglima et al., 1981; Levels, 1988). The factor produced by the adult $N$. guentheri did not affect the development of a closely related non-annual killifish Aphyosemion 
australe when the embryos were incubated together. However, incubation of annual killifish embryos with adults of different non-annual fish species resulted in arrest at diapause I and II, though the effect was diminished. Taken together this evidence suggests that some control over the initiation of embryonic diapause I and II is exercised through a specialized sensor or increased sensitivity in annual killifish to a common factor produced by fish. The decreased effect of this common factor produced in non-annual adults suggests that adult annual killifish may produce these compounds at higher levels. Alternately, it may be less easily degraded and/or metabolized, is specialized, or a combination of these factors (Inglima et al., 1981). Another study found that homogenates of ovary, and to a lesser magnitude testis, prolonged dispersion-reaggregation in $N$. korthause, without affecting incidence of diapause II. Embryo homogenates also inhibited development, though the effect was lost in homogenates prepared from hatchling fish. Boiling the homogenates increased the magnitude of the inhibitory effect by an unclear mechanism (Levels, 1988). Further work found that the factor prolonging dispersion-reaggregation was a polar, hydrophilic agent, although size determination was inconclusive. Interestingly, the apolar and hydrophobic purification fractions from adult ovary also contained a bioactive substance that in this case inhibited diapause II (Levels, 1988).

The biochemical nature of the substance inhibiting diapause II led the researchers to assess the influence of steroid hormones on the different stages of embryonic diapause. Embryonic incubation in progesterone spiked media $(>4 \mu \mathrm{g} / \mathrm{ml})$ significantly increased the duration of dispersion-reaggregation. All hormones tested 
had an inhibitory effect on diapause II, although only $\beta$-estradiol had an effect at concentrations as low as $0.001 \mu \mathrm{g} / \mathrm{ml}$. Significantly, of the hormones tested only $\beta$ estradiol and testosterone had inhibitory effects on diapause II without resulting in developmental anomalies in treated embryos (Levels, 1988).

Hormones are very likely candidates for regulation of diapause in annual killifish, especially considering their importance in regulating dormancy in other systems. Regulation of dormancy in nematodes is an excellent example. Dormancy in dauer stage larvae of the nematode $C$. elegans, is regulated through the insulin signaling cascade via the insulin receptor-like protein encoded by the gene Daf-2 (Kimura et al., 1997). The endocrine system in C. elegans integrates environmental and endogenous signals to affect responses that can include the decision to enter or bypass the dauer stage diapause (Tatar et al., 2003; Gerisch and Antebi, 2004). In stressful environments, the Forkhead transcription factor, DAF-16, remains unphosphorylated, localizes to the nucleus, and affects downstream gene regulation resulting in induction of the dauer dormancy. In beneficial environments this factor is phosphorylated, which favors a cytoplasmic localization and thus the absence of DAF-16 induced gene expression (Henderson and Johnson, 2001; Ogg et al., 1997). Evidence suggests that this pathway is mediated by Daf-9, a cytochrome P450 steroidogenic hydroxylase that produces a hormone integrating the system (Gerisch and Antebi, 2004). Further studies have identified Daf-9 as one of the most important aspects of the biosynthetic pathway involved in the production of the lipophilic steroid hormone involved in the dauer stage, although hormone production is affected 
through a multistep process (Rottiers et al., 2009). Daf-9 produces steroidal acid ligands (dafachronic acids) that when present inactivate the nuclear hormone receptor DAF-12; when ligands are absent dauer formation is initiated (Martin et al., 2010). Hormones clearly play an important role in the regulation of diapause in other systems as well. Peptidergic neurons in the subeosophageal ganglion of the silkworm, Bombyx mori, produce diapause hormone (DH), the initiator of embryonic diapause (Sato et al., 1993). Diapause hormone consists of 24 amino acid residues amidated at the carboxyl terminal, with a molecular weight of 2645 daltons, (Imai et al., 1991), encoded in a polyprotein precursor mRNA that also encodes four other hormones (Sato et al., 1993). This hormone acts on the ovaries, initiating gene transcription that ultimately results in the accumulation of glycerol and sorbitol, characteristics only of eggs in embryonic diapause (reviewed in Yamashita, 1995). Duration of diapause is regulated through the ERK and P38 MAP kinase cascades (Fujiwara et al., 2006). Activation of these cascades through extended low temperature terminates embryonic diapause (Iwata et al., 2005).

In the fleshfly, Sarcophaga crassipalpis, a failure to synthesize ecdysone is thought to arrest development. Concurrently, rhythmic pulses of juvenile hormone $(\mathrm{JH})$ herald the onset of embryonic diapause. These $\mathrm{JH}$ pulses continue through developmental arrest, and a pulse directly precedes the increase in molting hormone that terminates embryonic diapause (Walker and Denlinger, 1980). The interplay of these hormones modifies their physiological effects, possibly in a regulatory fashion; this is evidenced by changes in duration and/or onset of developmental arrest, under 
exogenous hormone treatments at different developmental time points (Denlinger, 1976; Zdarek and Denlinger, 1975). High temperature may also terminate diapause, through the ERK/MAP kinase cascade (Fujiwara and Denlinger, 2007), a likely candidate for the control of embryonic diapause in A. limnaeus. Furthermore, as in annual killifish, maternal photoperiod affects diapause incidence in progeny of the fleshfly S. bullata, although by an unknown mechanism (Rockey et al., 1989). Global control of major life history transitions are often controlled by hormones; these compounds are structurally well conserved and powerful effector molecules that act to coordinate organism-wide responses. The role of hormones has been suggested in the regulation of embryonic diapause in annual killifish, and exogenous hormone treatment found to significantly affect developmental pathways in embryos of $N$. korthause (Levels, 1988). To elucidate factors involved in the control of embryonic diapause in A. limnaeus, I have investigated the role of steroid hormones in both maternal and embryonic control of diapause, and screened for changes in gene expression using a cDNA microarray approach. I hypothesize that hormone levels in escape and diapause embryos will differ through early development leading up to dormancy, and the hormonal environment in utero will influence the decision to bypass or enter embryonic diapause. This variation in hormone levels will be accompanied by differences in hormone receptors and their downstream gene products. Through these processes, ultimate developmental pathway will be determined, and the suite of metabolic and biochemical traits essential to different trajectories effected. 


\section{Materials and Methods}

\section{Husbandry of Adults and Collection of Embryos}

Experimental animals were obtained from an in-house aquatic facility, as previously described (Podrabsky, 1999). Briefly, breeding pairs of A. limnaeus were housed in $3.8 \mathrm{~L}$ aquaria attached to shared sumps (21 aquaria per sump). Pairs were kept separate using an internal divider until spawning. Water was changed twice daily (25\% each time) weekdays, and once daily on weekends (25\%), using City of Portland tap water that was charcoal filtered, UV treated, and supplemented with salt (Coralife synthetic sea salt, $0.115 \%$ ). Animals were fed on the same schedule as the water changes, primarily on Bio-pure frozen bloodworms (Hikari-US Koi, LLC, Cat\# 30221). Chopped red wiggler worms (Timberline Live Pet Food) were fed on days immediately prior to spawning. Photoperiod was maintained on a $14 \mathrm{hr}$ of light and $10 \mathrm{hr}$ of dark (14:10 L:D) regimen, and ambient temperature kept at $\approx 26^{\circ} \mathrm{C}$. System water temperatures normally ranged between $24-26^{\circ} \mathrm{C}$.

Embryos were collected twice-weekly, also as described in Podrabsky (1999), and maintained in embryo medium $\left(10 \mathrm{mM} \mathrm{NaCl}, 0.1424 \mathrm{mM} \mathrm{KCl}, 2.15 \mathrm{mM} \mathrm{MgCl}_{2}\right.$, $0.0013 \mathrm{mM} \mathrm{MgSO}_{4}, 0.792 \mathrm{mM} \mathrm{CaCl}$ in Nanopure water) containing methylene blue $(0.0001 \%)$ until $4 \mathrm{dpf}$ to inhibit fungal and bacterial growth. The embryos were then bleached in a mild sodium hypochlorite solution $(0.03 \%, 2 \times 5 \mathrm{~min})$ as described in Podrabsky (1999). To ensure neutralization of the bleach, the embryos were rinsed in 
$0.005 \%$ sodium thiosulfate for $10 \mathrm{~min}$. Following the bleaching treatment, embryos were transferred into embryo medium containing gentamicin sulfate $\left(10 \mathrm{mg} \mathrm{L}^{-1}\right)$ to inhibit bacterial growth. Embryos were maintained in $100 \mathrm{~mm}$ x $15 \mathrm{~mm}$ petri plates (VWR, Cat\#25384-342) at densities of 50-100 embryos per dish. Embryo medium was changed daily for the first four dpf, and then 6 times a week following. Dishes of embryos were incubated at constant temperature $\left(25\right.$ or $30^{\circ} \mathrm{C}$ depending on the experiment) in the dark (VWR growth chambers, model 2015).

Developmental pathway of embryos at $25^{\circ} \mathrm{C}$ was scored at $18-22 \mathrm{dpf}$ with bright phase microscopy (Leica DMIRB inverted microscope). The proportion of escape embryos was quantified through a representative sample of each female's clutch allowed to develop normally (10-12 embryos). Females were monitored for multiple spawning events (>3) before tissue harvesting.

\section{Extraction of Steroid Hormones}

Adult tissues

Adult fish were anesthetized in ice water for several minutes, followed by cervical dislocation. Ovary tissues were quickly harvested, placed into $14 \mathrm{ml}$ polypropylene tubes, and flash frozen in liquid nitrogen. Tissues were stored at $-80^{\circ} \mathrm{C}$ until immediately prior to hormone extraction. Hormones were extracted from whole tissues by homogenization in $5 \mathrm{ml}$ of ice cold $100 \%$ ethanol (Sigma-Aldrich, 
Cat\#493546) using an IKA rotary homogenizer (IKA Works, Ultra-Turrax S10). After homogenization, the dispersing element was rinsed into the sample tube with an additional $2 \mathrm{ml}$ absolute ethanol. Samples were spun at $0^{\circ} \mathrm{C}$ in a refrigerated centrifuge (Sorvall, Superspeed RC2-B) for $10 \mathrm{~min}$ at $3020 \mathrm{x} \mathrm{g}$. The supernatant was collected into baked borosilicate tubes, capped, and stored at $-20^{\circ} \mathrm{C}$ until hormone quantification.

Whole Blood was collected from adult fish through an incision made at the caudal peduncle, and whole blood from the caudal vein and artery collected into micro hematocrit tubes $(75 \mu l$, heparinized). The tubes were chilled on ice for a maximum of one hour before centrifugation for $5 \mathrm{~min}$ at full speed in a hematocrit centrifuge (Adams Autocrit). The plasma fraction was isolated, weighed, and flash frozen in liquid nitrogen. Plasma samples were stored at $-80^{\circ} \mathrm{C}$ until extraction and analysis.

\section{Whole Embryos}

Embryos were collected onto a mesh screen using a wide-mouth transfer pipette, blotted dry with kimwipes, weighed, and then flash frozen in liquid nitrogen. Frozen embryos were stored at $-80^{\circ} \mathrm{C}$ until immediately prior to processing. Embryos were homogenized and frozen by the same protocol as previously described for tissues, in either $5 \mathrm{ml}$ absolute ethanol for groups larger than 40, or in $3 \mathrm{ml}$ for groups 
of 20, with the dispersing element rinsed in both cases with $2 \mathrm{ml}$ of absolute ethanol. 


\section{Isolation and Quantification of Steroid Hormones}

Steroid hormone levels were quantified according to standard protocols by the Endocrine Technology and Support Core Lab at the Oregon National Primate Research Center/Oregon Health \& Science University (Rasmussen et al., 1984). Briefly, ethanol extracts were dried under forced air, then extracted with $300 \mu 1$ of $0.1 \%$ bacto-gelatin in $1 \mathrm{X}$ PBS and $5 \mathrm{ml}$ redistilled diethyl ether to separate aqueous and ether soluble materials. The ether soluble fractions were decanted and then dried under forced air. The extract was then re-dissolved in $200 \mu \mathrm{l}$ of column solvent (Hexane:benzene:methanol $=62: 20: 13$ ). Steroid hormones were separated by column chromatography in a 1 x $6 \mathrm{~cm}$ glass column containing $1 \mathrm{~g}$ Sephadex LH-20, and individual hormone fractions collected and dried under forced air. Each hormone was quantified by specific radioimmunoassay (Antibodies: $17 \beta$-estradiol- Dr. G.

Niswender, GDN\#344; Androstenedione- ICN Pharmaceuticals, 07109016;

Testosterone- W. Ellinwood, WEE-23; Progesterone- Sandoz Pharmaceuticals, Surve 12; Corticosterone-ICN Pharmaceuticals, 07120017). Final values were corrected to account for recovery efficiency as determined by addition of a radioactive tracer to the sample (Tracers: $17 \beta$-estradiol-NEN Radiochemicals, NET317250UC; Androstenedione-NEN Radiochemicals, NET469250UC; Testosterone-NEN Radiochemicals, NET370250UC; Progesterone-NEN Radiochemicals, NET381250UC; Corticosterone-NEN Radiochemicals, NET399250UC). Recovery efficiencies were typically $60-80 \%$. The limit of sensitivity for $17 \beta$-estradiol was 3 
$\rho g$ / assay tube, and $5 \mathrm{\rho g} /$ assay tube for androstenedione, progesterone, corticosterone, and testosterone.

\section{Treatment of Embryos with Exogenous Steroid Hormones}

$17 \beta$-estradiol (E8875-1g) and cortisol (hydrocortisone; H4001-1g) were purchased from Sigma-Aldrich. Hormones were suspended in absolute ethanol, and added to embryo medium containing 1\% dimethyl sulfoxide (DMSO). DMSO was used as a permeabilizing agent, to improve hormone movement into the embryos. Concentrations of $1,5,10$, and $20 \mu \mathrm{g} / \mathrm{ml}$ of hormone were prepared. Ethanol was supplemented to control as well as hormone treated embryos to an equal total $(0.133 \%)$. Embryos were raised at $25^{\circ} \mathrm{C}$, bleached at day 4 as previously described, then transferred in groups of 20 embryos per well to 6-well plates containing hormone medium at $6 \mathrm{dpf}$. The hormone medium was changed every other day, and the embryos scored as entering diapause II or as escape embryos at $18 \mathrm{dpf}, 12$ days following the commencement of hormone treatment.

Steroid hormone levels were quantified at 6, 10, 14, and 18 days following the initiation of exogenous hormone treatments. At the time of harvest, the embryos were transferred into clean culture plates, rinsed 3 times with nanopure water, soaked in nanopure water for $10 \mathrm{~min}$ to remove hormones from the perivitelline space, and then rinsed again 3 times. Embryos were pipetted onto a mesh screen, blotted dry, and flash frozen in liquid nitrogen. Hormones were extracted as previously described. Dechorionated embryos were treated and rinsed as previously described, then 
deposited onto mesh screens inserted into $1 \mathrm{ml}$ syringes. The syringe-screen assembly was placed into $15 \mathrm{ml}$ falcon tubes, the plunger inserted to crush the embryos, and the assembly subjected to centrifugation at $0^{\circ} \mathrm{C}$ (Sorvall, Superspeed RC2-B) for $10 \mathrm{~min}$ $(3000 \mathrm{x} g)$. The dechorionated embryo fraction was flash frozen in liquid nitrogen and then extracted in ice cold $100 \%$ ethanol as previously described.

\section{Steroid Hormones During Normal Development}

Levels of $17 \beta$-estradiol (E2) and androstenedione (A4) were quantified during early development in embryos of A. limnaeus reared at both 25 and $30^{\circ} \mathrm{C}$. Embryos were sampled at the following developmental stages at both temperatures: full epiboly, 5-10 somite, 14-16 somite, and 25-30 somite. Additionally, embryos were sampled directly after fertilization (newly fertilized), and both during diapause and four dpf in embryos incubated at $25^{\circ} \mathrm{C}$. At full epiboly the blastomeres have expanded to completely encircle the yolk sack as amoeboid cells, but remain relatively large and have not yet undergone multiple rounds of mitosis (stage 19; Wourms, 1972a); diapause I may be initiated directly following this stage. 5-10 somite embryos have a fully developed embryonic axis without a functional heart (stage 29-30; Wourms, 1972a). At this developmental stage, escape embryo production sharply increases from background levels under $30^{\circ} \mathrm{C}$ incubation (Podrabsky et al., 2010). 14-16 somite embryos have a beating heart and a widened cephalic region (stage 30-31; Wourms, 1972a). This is the final stage preceding 
morphological divergence between embryos incubated at 25 or $30^{\circ} \mathrm{C}$, though the majority of embryos incubated at $30^{\circ} \mathrm{C}$ until this stage will follow the escape trajectory (Podrabsky et al., 2010). By the 25-30 somite stage embryos at the different incubation temperatures have diverged sharply, both morphologically and physiologically, with embryos incubated at $25^{\circ} \mathrm{C}$ showing comparable morphological development only four days post-diapause II at approximately 45 somites. Due to the effects of temperature, embryos reared at 25 and $30^{\circ} \mathrm{C}$ reached these developmental stages at different times. Newly fertilized embryos were harvested within 4 hours of being spawned, and fertilization (formation of the perivitelline space) validated under a dissecting scope. Diapause II embryos were harvested approximately 4-5 days following initiation of diapause and had heart rates lower than 10 beats per minute. Embryos incubated at $30^{\circ} \mathrm{C}$ were transferred from 25 to $30^{\circ} \mathrm{C}$ at approximately 18 hours post fertilization, and cared for by identical protocols as previously detailed. Embryonic development was monitored using bright phase microscopy (Leica DMIRB inverted microscope).

\section{Extraction of RNA}

Adult fish were anesthetized in ice water for several minutes, followed by cervical dislocation. Adult female tissues were quickly harvested, placed into $14 \mathrm{ml}$ polypropylene tubes, and flash frozen in liquid nitrogen. Embryos were collected onto a mesh screen using a wide-mouth transfer pipette, blotted dry with kimwipes, 
weighed, and then flash frozen in liquid nitrogen. Embryonic development and escape embryo proportions were monitored as previously described. Samples were taken from newly fertilized embryos (within four hours of spawning), and at 14-16 somite stages in embryos reared at 25 and $30^{\circ} \mathrm{C}$. Liver and ovary tissues were sampled from spawning females. Frozen embryos and adult tissues were stored at $-80^{\circ} \mathrm{C}$ until immediately prior to processing.

RNA was extracted from frozen tissue and embryo samples by homogenization in 10 volumes (by weight) for tissues, and 20 volumes for embryos, of Trizol reagent (Invitrogen, Cat\#15596-018) using an IKA rotary homogenizer (IKA Works, Ultra-Turrax S10) in $14 \mathrm{ml}$ polypropylene tubes. The samples were homogenized in Trizol until they had an even consistency, then placed at room temperature for at least $15 \mathrm{~min}$, allowing nucleoprotein complexes to dissolve. Samples were subjected to centrifugation at $10,000 \times \mathrm{g}\left(8-10^{\circ} \mathrm{C}\right)$ in a refrigerated centrifuge (Sorvall, Superspeed RC2-B) for 30 min to pellet insoluble material. The supernatant was decanted into a fresh tube, 0.2 volumes of $100 \%$ chloroform added per initial $\mathrm{ml}$ of Trizol used, then the sample gently vortexed until the phases had completely mixed. The homogenate was then subjected to centrifugation at $10,000 \mathrm{x}$ $g\left(8-10^{\circ} \mathrm{C}\right)$ for 20 min to separate the aqueous and organic phases. The aqueous phase (which contains the RNA) was carefully removed and placed into a fresh $14 \mathrm{ml}$ tube on ice. The RNA was precipitated using a high salt precipitation which consisted of adding 0.25 volumes of high salt precipitation solution $(0.8 \mathrm{M}$ sodium citrate and 1.2 M sodium chloride) and 0.25 volumes $100 \%$ isopropanol to the supernatant for each 
$\mathrm{ml}$ of Trizol originally used for the homogenization. The samples were placed at $20^{\circ} \mathrm{C}$ overnight to allow the RNA to precipitate, then centrifuged at $10,000 \times \mathrm{x}$ ( 8 $10^{\circ} \mathrm{C}$ ) for 30 min to pellet the RNA. The supernatant was removed without disturbing the RNA pellet. The pellet was washed twice with $2 \mathrm{ml} 60 \%$ ethanol followed each time by centrifugation at $10,000 \times \mathrm{g}$ for $20 \mathrm{~min}$. The ethanol was removed and the pellet was allowed to air dry for 5 to 10 min until clear. The isolated total RNA was covered in nuclease free water $(20-100 \mu \mathrm{l})$ and heated to $55^{\circ} \mathrm{C}$ for $5 \mathrm{~min}$ to aid in solubilization. Following resuspension, the RNA samples were kept either frozen or on ice at all times. The amount of total RNA was quantified by UV absorbance at 260 $\mathrm{nm}$ using the extinction coefficient of $40 \mu \mathrm{g} / \mathrm{OD}_{260}$. RNA purity was assessed by determining the ratio of absorbance at 260 and $280 \mathrm{~nm}$ (Range: 1.43-2). RNA quality was validated by visualization on $1 \%$ agarose gels stained with ethidium bromide (1X TBE) $(100 \mathrm{~V}$, run time $=15 \mathrm{~min})$. RNA samples requiring concentration were precipitated at $-20^{\circ} \mathrm{C}$ overnight with 0.1 volume $3 \mathrm{M}$ sodium acetate and 2.5 volumes $100 \%$ ethanol. After precipitation, RNA was pelleted by centrifugation at $12,000 \times \mathrm{x}$ for $20 \mathrm{~min}$ and the samples washed twice with $2 \mathrm{ml} 80 \%$ ethanol followed by centrifugation at 12,000 x $g$ for $20 \mathrm{~min}$ (Sorvall, Superspeed RC2-B). The ethanol was then removed, the RNA pellets allowed to air-dry, and the samples resuspended by the previously listed method. Aliquots of the RNA sample were frozen in separate tubes to avoid multiple freeze thaws cycle for each sample. 


\section{Amplification of Total RNA}

Embryonic RNA samples were amplified using the MessageAmp 2 aRNA amplification kit according to the manufacturer's instructions (Applied Biosystems, Cat\#AM1751), based on previously published techniques (Van Gelder et al., 1990). Full reactions were run on all day 0 embryo samples ( $1 \mu \mathrm{g}$ input total RNA), and 1/2 reactions performed on 14-16 somite embryo samples ( $0.5 \mu \mathrm{g}$ input total RNA, all steps $1 / 2$ total volume of following protocol). The average ratio of absorbance measured at 260 versus $280 \mathrm{~nm}$ for each RNA sample used was 1.7 (Range: 1.4-2.0). All reagents used were provided in the amplification kit unless otherwise noted.

Single-stranded cDNA (ss cDNA) was prepared from 0.5-1.0 $\mu$ g of total RNA in a final volume of $20 \mu 1(11 \mu 1$ RNA + nuclease free water, $1 \mu 1 \mathrm{~T} 7$ Oligo(dT) primer, $2 \mu 1$ 10X first strand buffer, $4 \mu 1$ dNTP mix, $1 \mu 1$ RNase inhibitor, $1 \mu 1$ Arrayscript reverse transcriptase). The reactions were incubated at $16^{\circ} \mathrm{C}$ for 2 hours in a thermocycler (Applied Biosystems, Gene Amp 2700) with the thermocycler lid open. After 2 hours the tubes were removed, placed on ice, then the contents added to $250 \mu 1$ of cDNA binding buffer. The diluted ss cDNA was applied to a DNA filter cartridge and centrifuged at $10,000 \times \mathrm{g}$ for $1 \mathrm{~min}$ to bind the DNA to the column material. The column was washed once with $500 \mu 1$ wash buffer and centrifuged again twice, each time at $10,000 \times \mathrm{g}$ for $1 \mathrm{~min}$. The filter cartridges were then transferred to clean tubes and the cDNA eluted from the columns with $18 \mu 1$ of nuclease free water (preheated to $55^{\circ} \mathrm{C}$ ) by centrifugation at $10,000 \mathrm{x} g$ for $1 \mathrm{~min}$. The 
eluted cDNA was then amplified in a reaction mixture containing $18 \mu \mathrm{l}$ of ss cDNA and $24 \mu 1$ of IVT master mix (IVT master mix; $4 \mu 1$ T7 ATP, $4 \mu 1$ T7 CTP, $4 \mu 1$ T7 GTP, $4 \mu 1$ T7 UTP, $4 \mu 1$ T7 10X Reaction buffer, $4 \mu 1$ T7 enzyme mix) incubated at $37^{\circ} \mathrm{C}$ for 14 hours in a thermocycler (Applied Biosystems, Gene Amp 2700). After the incubation, aRNA samples were brought to a final volume of $100 \mu$ with nuclease free water, transferred to new nuclease free tubes, and mixed with $350 \mu 1$ of aRNA binding buffer. The aRNA was mixed with $250 \mu 1$ of absolute ethanol and applied onto an aRNA filter cartridge. The samples were centrifuged at 10,000 x $g$ for $1 \mathrm{~min}$ to allow the aRNA to bind to the column. The column was then washed with $650 \mu 1$ of wash buffer followed by 2 centrifugations at $10,000 \times \mathrm{g}$ for $1 \mathrm{~min}$ each to remove residual wash buffer. The aRNA was eluted in $200 \mu \mathrm{l}$ of nuclease free water (preheated to $55^{\circ} \mathrm{C}$ ) by centrifugation at $10,000 \times \mathrm{g}$ for $1 \mathrm{~min}$. RNA yield was quantified by UV absorbance at $260 \mathrm{~nm}$ as described above (Shimadzu Pharma Spec UV-1700). Average yield for newly fertilized embryo amplification samples was 86 $\mu \mathrm{g}$ RNA with an average 260/280 nm ratio of 1.99 (Range: 1.7-2.15). Average yield for 14-16 somite embryos was $81.5 \mu \mathrm{g}$ RNA with an average $260 / 280 \mathrm{~nm}$ ratio of 2.08 (Range: 1.6-2.2).

\section{Synthesis of Fluorescent Probes}

Production of single-stranded amino-allyl cDNA 
Fluorescently labeled probes were synthesized as previously described using Cy3 and Cy5 as the dyes (Podrabsky and Somero, 2004). Each experimental sample was competitively hybridized against a pooled reference RNA sample consisting of total RNA from liver and ovary tissues. $15 \mu \mathrm{g}$ of total RNA (aRNA from embryos, total RNA for adult tissues) was used for the synthesis of all probes. Single-stranded cDNA was synthesized in a reaction mixture consisting of $8 \mu \mathrm{l}$ of RNA + nuclease free water, combined with primers $\left(1 \mu \mathrm{l}\right.$ of $5 \mu \mathrm{g} / \mu \mathrm{l}$ oligo $\mathrm{dT}_{15} \mathrm{VN}, 1 \mu \mathrm{l}$ of $2.8 \mu \mathrm{g} / \mu \mathrm{l}$ random hexamer; Integrated DNA Technologies Inc.) $4 \mu \mathrm{l}$ of $5 \mathrm{X}$ reverse transcriptase buffer, 1 Hl 20X dNTP/aa-dUTP (20X: 10 mM dATP, 10 mM dCTP, 10 mM dGTP, 5 mM dTTP, 5 mM aa--dUTP), $2 \mu 1100$ mM DTT, $2 \mu$ reverse transcriptase (40 units), and $1 \mu 1$ nuclease free water for a total volume of $20 \mu \mathrm{l}$. The RNA and primers were first mixed and heated at $70^{\circ} \mathrm{C}$ for $10 \mathrm{~min}$ and then placed directly on ice. The rest of the components were then added and the samples incubated at $42^{\circ} \mathrm{C}$ for $90 \mathrm{~min}$ in a thermocycler (Applied Biosystems, GeneAmp 2700). After incubation the RNA was degraded by adding $6.67 \mu 11 \mathrm{M} \mathrm{NaOH}$, and $6.67 \mu 10.5 \mathrm{M}$ EDTA and heating the samples to $65^{\circ} \mathrm{C}$ for $15 \mathrm{~min}$. The $\mathrm{pH}$ of the solution was neutralized by the addition of $17 \mu \mathrm{l}$ of $1 \mathrm{M}$ Tris $(\mathrm{pH}=7.5)$. The ss cDNA was purified using a Qiagen PCR purification kit according to the manufacturer's instructions with the exception that $80 \%$ ethanol was used instead of the wash buffer provided (QIAquick PCR purification kit, Qiagen, 28104). Single-stranded cDNA was eluted by two applications of $30 \mu \mathrm{l}$ of nuclease free water $(\mathrm{pH}>7)$ warmed to $37^{\circ} \mathrm{C}$ followed by centrifugation at $10,000 \times \mathrm{g}$ for $1 \mathrm{~min}$ following each application. The eluted ss 
cDNA was dried in a speedvac concentrator (ThermoSavant, SpeedVac Plus SC210A).

Fluorescent Labeling of Probes with Cy Dyes

The amino-allyl labeled cDNA pellet was resuspended in $5 \mu 10.1 \mathrm{M}$ $\mathrm{NaHCO}_{3}$, then combined with $5 \mu \mathrm{l}$ of Cy3 (Amersham Biosciences, Q13104) or Cy5 mono-reactive dye (Amersham Biosciences, Q15104) dissolved in DMSO (80 $\mu 1$ per tube dye), and incubated in the dark at room temperature for 1 hour. The reaction was stopped by a $15 \mathrm{~min}$ incubation (at room temperature) following the addition of $4.5 \mu \mathrm{l}$ of $4 \mathrm{M}$ hydroxylamine. Each experimental sample was then combined with a reference sample labeled with the opposite colored probe. The probe mixtures were purified using a Qiaquick PCR purification kit according to the manufacturer's instructions (QIAquick PCR purification kit, Qiagen, 28104). The labeled ss cDNA probes were eluted from the column with two subsequent applications of $30 \mu \mathrm{l}$ of buffer $\mathrm{EB}$ warmed to $37^{\circ} \mathrm{C}$ followed by centrifugation at $10,000 \mathrm{x} g$ for $1 \mathrm{~min}$. The labeled probes were brought to a final volume of $80 \mu \mathrm{l}$ and a final concentration of 3X SSC, $0.75 \mathrm{mg} / \mathrm{ml}$ tRNA (Sigma, R8508-1ML) and $25 \mathrm{mM}$ HEPES ( $\mathrm{pH}=7$ ), and $0.25 \%$ SDS. Immediately prior to use the probes were heated for $2 \mathrm{~min}$ at $100^{\circ} \mathrm{C}$ in a heat block and allowed to cool at room temperature for 1-2 min. The contents were collected into the bottom of the tube by a brief centrifugation $(1.5 \mathrm{x} g$ for $10 \mathrm{sec})$. 


\section{Hybridization of Fluorescent Probes}

Microarray hybridizations were conducted on cDNA microarray slides, fabricated largely as previously published (Podrabsky and Somero, 2004). The arrays were printed using a GeneMachines Omnigrid Accent robotic array printer using a 32 pin configuration, with $150 \mu \mathrm{m}$ spot spacing, and over 16,000 spots total. Microarrays were pre-processed according to standard protocols (Podrabsky and Somero, 2004). Immediately prior to use, the microarray slides were prepared for probe application by immersion in prehybridization blocking solution for at least 1 hour (blocking solution; $5 \mathrm{X}$ SSC, $1 \%$ Bovine serum albumin, $0.1 \%$ SDS), then washed twice in nanopure water ( 2 min each), and dried by centrifugation at $500 \mathrm{x} g$ for 9 min (Eppendorf, 5810R). Probes were applied to the microarray under a LifterSlip cover slip (Erie Scientific Co., 25x541-2-4901) placed over the array. The slides were quickly transferred to an array hybridization chamber (Genetix, x2530) that was preheated and humidified at $65^{\circ} \mathrm{C}$. Probes were allowed to hybridize overnight (12-14 hours) at $65^{\circ} \mathrm{C}$ in a hybridization oven (VWR International, model 5420). Following hybridization, the coverslip was removed after submersion of the slides into a wash buffer containing 0.6X SSC and $0.03 \%$ SDS. The slides were washed briefly in a second container of this wash solution followed by a brief wash in 0.06X SSC and finally in nanopure water. At each washing step the slides were gently plunged up and 
down 5-10 times. Slides were dried by centrifugation at $500 \mathrm{x} g$ for $9 \mathrm{~min}$ at $25^{\circ} \mathrm{C}$ (Eppendorf, 5810R).

\section{Fluorescence Quantification and Data Analysis}

The microarray slides were scanned on an Axon Genepix 4000B microarray scanner, using GenePix Pro 5.1 software, at both 635 and $532 \mathrm{~nm}$ wavelengths. Laser power was maintained at $100 \%$, and normalized counts of the intensity distribution balanced using the PMT gain. Array spots were visually checked, and aberrant spots flagged and disqualified from analysis. Fluorescent intensity was quantified and used to calculate the median ratio of $\mathrm{Cy} 3$ to $\mathrm{Cy} 5$ for each spot. The background-corrected ratio of the median intensity for each spot was imported into GeneSpring GX 10.0 software. The overall ratios for each dye were normalized globally and changes in gene expression analyzed using an unpaired T-Test with asymptotic p-value computation (Benjamini-Hochberg multiple testing corrections, $\mathrm{p}<0.05$ ). Clones more than 2 fold differentially regulated, or with elevated significance values in relation to the other clones, were included in the results. It should be noted that the analysis presented here is of a fraction of the total genes identified as being differentially regulated in different tissues and at different developmental time points. 


\section{DNA sequencing}

The E. coli clones (Invitrogen, DH10B) containing the plasmids of interest (Clontech, p $\lambda$ TriplEx2) were removed from a $-80^{\circ} \mathrm{C}$ freezer and thawed at $4-6^{\circ} \mathrm{C}$ (for cDNA library preparation see Podrabsky and Somero, 2004). The clones of interest were transferred from the cDNA library using $10 \mu \mathrm{l}$ pipet tips, into $200 \mu \mathrm{l}$ of LB broth containing $10 \%$ glycerol and Ampicillin $\left(50 \mathrm{mg} \mathrm{L}^{-1}\right)$ in 96-well Costar round bottom plates (Fisher, 07-200-105). The plates were wrapped in plastic wrap, placed into tupperware with moist paper towels, and grown statically at $37^{\circ} \mathrm{C}$ for 48 hours. After the first growth period, the clones were transferred to $200 \mu \mathrm{l}$ of fresh LB/glycerol medium using a 96 pin replicator tool (V\&P Scientific Inc., 250520) and allowed to grow for $12 \mathrm{hr}$ at $37^{\circ} \mathrm{C}$. The plates were sealed with aluminum adhesive seals (VWR, 100845-646) and frozen at $-80^{\circ} \mathrm{C}$. The $96-$ well plates were packed on dry ice in styrofoam coolers, and shipped overnight to Beckman-Coulter Genomics (Danvers, MA). The samples were sequenced unidirectionally with the 5'TriplEx2 primer (5 'CTCCGAGATCTGGACGAGC 3'), with an average of 678 high quality base pairs per read. The putative identity of each clones was assessed using the BlastX multiple alignment tool (http://www.ncbi.nlm.nih.gov/blast/Blast.cgi?PAGE=Translations\&PROGRAM=blas tx) against the GenBank non-redundant protein database. 


\section{Graphs and statistical analyses}

Statistical analyses were performed using Prism 4.0 software (Graph Pad). Correlation, analysis of variance (ANOVA), Kruskal-Wallis, Mann Whitney, and $t$ tests were used where appropriate. Tukey's multiple comparison test or Dunn's multiple comparison test were used post hoc to determine significantly different groups. P-values $<0.05$ were considered significant. Fractions of escape embryos were transformed prior to statistical analysis by taking the arc sine of the square root of the fraction $(\operatorname{ArcSin} \sqrt{X})$. 


\section{Results}

\section{Estrogen levels are higher in younger compared to older females}

Investigation of $17 \beta$-estradiol levels in females of different age classes indicated higher levels in ovarian tissue and plasma in younger females (Fig. 1A,B). This pattern is similar to the pattern observed for production of escape embryos in females of different age classes, where the youngest females produce the highest proportion of escape embryos. The production of escape embryos decreases through maternal life, with the lowest level of escape production in the oldest females. The youngest age class (90-105 days old) had mean $17 \beta$-estradiol levels in the ovary of $12,382 \rho \mathrm{g} / \mathrm{g} \pm 1,452$ and $11,636 \rho \mathrm{g} / \mathrm{ml} \pm 1,739$ in plasma. The mid-age class had intermediate $17 \beta$-estradiol values (while not significantly different from older females) of $6,323 \rho \mathrm{g} / \mathrm{g} \pm 1,490$ in ovarian tissue, and 5,250 $\mathrm{\rho g} / \mathrm{ml} \pm 1,541$ in plasma. The oldest age class had the lowest $17 \beta$-estradiol levels, with 5,088 $\rho g / g \pm 873$ in ovary, and 3,204 $\rho \mathrm{g} / \mathrm{ml} \pm 744$ in plasma. The results indicate a decline of $59 \%$ in ovarian $17 \beta$-estradiol concentrations from young to old females, and a decline of $73 \%$ in plasma levels.

\section{Hormone panels in ovaries of young females}

No significant differences in ovarian hormone levels were discovered between females producing different ratios of escape embryos (Fig. 2). However, there was a trend towards higher androstenedione values in females producing less than $8 \%$ 
escape embryos compared to those producing a high proportion of escape embryos $(2,952 \rho g / g \pm 628$ vs $1,073 \rho g / g \pm 603, p=0.0854, \mathrm{t}=1.923, \mathrm{df}=10)$. The mean $17 \beta$ estradiol level in young females was $11,551 \rho \mathrm{g} / \mathrm{g} \pm 1299$, levels of progesterone averaged $497 \rho \mathrm{g} / \mathrm{g} \pm 84$, androstenedione levels were $2314 \rho \mathrm{g} / \mathrm{g} \pm 524$, and testosterone levels were $814 \rho \mathrm{g} / \mathrm{g} \pm 217$. Interestingly, ratios of $17 \beta$-estradiol to testosterone and progesterone exhibit a high degree of correlation with the proportion of escape embryos produced (Fig.3). Increased $17 \beta$-estradiol/testosterone and $17 \beta$ estradiol/progesterone ratios are associated with higher proportions of escape embryos (Pearson $\mathrm{r}=0.768, \mathrm{p}=0.0035$; Pearson $\mathrm{r}=0.463, \mathrm{p}=0.1491$, respectively). It is important to note that in both cases the relationship is influenced by possible outliers. When these points are removed, the association between $17 \beta$-estradiol/testosterone is weakened although remains significant (Pearson $r=0.645, p=0.0323)$, and the correlation between $17 \beta$-estradiol/progesterone ratios and the production of escape embryos becomes significant (Pearson $\mathrm{r}=0.872$, $\mathrm{p}=0.0005$ ).

\section{Embryonic hormone levels}

There were no significant differences in hormone levels of newly fertilized embryos sampled from clutches with high (>85\%) and low (0\%) proportions of escape embryos produced (Fig. 4A,B). Therefore, hormone levels are presented as combined values for both escape and diapause groups. The mean hormone concentrations were: 0.9 pg/embryo $\pm 0.117 \beta$-estradiol, $1.3 \rho g$ /embryo \pm 0.2 corticosterone, $0.8 \rho \mathrm{g} / \mathrm{embryo} \pm 0.2$ progesterone, $0.4 \rho \mathrm{g} / \mathrm{embryo} \pm 0.1$ androstenedione, 
and $0.3 \rho g$ /embryo \pm 0.08 testosterone. As a function of tissue mass, the mean values are $379 \rho \mathrm{g} / \mathrm{g} \pm 25,542 \rho \mathrm{g} / \mathrm{g} \pm 92,311 \rho \mathrm{g} / \mathrm{g} \pm 82,177 \rho \mathrm{g} / \mathrm{g} \pm 63$, and $119 \rho \mathrm{g} / \mathrm{g} \pm 35$ respectively.

$17 \beta$-estradiol levels fall through early development, to a significantly lower level in $30^{\circ} \mathrm{C}$ embryos at the 5-10 somite stage (Fig. 5A: Two way ANOVA, p<0.05, $\mathrm{t}=2.925)$. Androstenedione values follow the same pattern as $17 \beta$-estradiol, although the drop is not significant (Fig. 5B). Hormone levels increase again following the 5 10 somite stage. This is the developmental period where $30^{\circ} \mathrm{C}$ treatment results in a steep increase in the production of escape embryos.

\section{Embryonic hormone treatments}

Exogenous treatment with $17 \beta$-estradiol and cortisol resulted in significantly increased numbers of escape embryos (Fig. 6AB). Incubation in $17 \beta$-estradiol caused the majority of embryos to follow the escape trajectory at concentrations above 5 $\mu \mathrm{g} / \mathrm{ml}$ (Kruskal-Wallis test $\mathrm{p}=<0.0001, \mathrm{KW}$ statistic=40.64) (Fig. 6A). The magnitude of this effect did not significantly change by increasing the treatment concentration from 5 to 10 or $20 \mu \mathrm{g} / \mathrm{ml}$ (escape percentages respectively: $64 \pm 7,75 \pm 7$, and $72 \pm 11$ ), suggesting a plateau effect (Fig. 6B). Cortisol treatment also significantly increased the percentage of embryos following the escape trajectory at concentrations above $5 \mu \mathrm{g} / \mathrm{ml}$ (One way ANOVA p<0.0001, F=26.24). However, increasing cortisol concentrations above this concentration had an additive effect on the number of escape embryos, unlike the plateau effect seen with $17 \beta$-estradiol treatment. 
$17 \beta$-estradiol treatment at levels above $1 \mu \mathrm{g} / \mathrm{ml}$ led to a saturation effect in embryos. Higher concentrations in treatment levels were not significantly different at most points during treatment (Fig. 7). Dechorionated control embryos had estradiol levels that were not statistically different than hormone levels in $25^{\circ} \mathrm{C}$ post-diapause II embryos (Fig. 8). Estradiol levels were slightly higher than in $30^{\circ} \mathrm{C} 25-30$ somite embryos, the stage developmentally closest to the dechorionated embryos. However, dechorionated embryos were sampled at $18 \mathrm{dpf}$ whereas $25-30$ somite embryos were sampled at 12-14 dpf. $17 \beta$-estradiol levels in estrogen-treated embryos were significantly higher than in controls ( $\mathrm{t}$-test $\mathrm{p}=0.0005, \mathrm{t}=10.48, \mathrm{df}=4)$. Androstenedione levels in estrogen treated embryos were also significantly higher than in controls $(\mathrm{p}=0.0018, \mathrm{t}=7.339, \mathrm{df}=4)$, indicating possible reverse aromatase activity. Removing the chorion dramatically changed the amount of estradiol detected in hormone treated embryos. Measured $17 \beta$-estradiol levels decreased by more than $99 \%$ after chorion removal, from an average of $38,532 \rho g$ to $129 \rho g$ per embryo.

\section{Differential Expression of Genes Associated with Escape Embryos}

A total of 146 genes were identified as differentially expressed in maternal tissues isolated from females producing escape embryos and embryos on an escape trajectory (see appendix for sequences). Sequences identified as differentially expressed are presented in figure 9 organized as a function of tissue. Of the total, 44 genes were identified as differentially expressed in escape embryos of A. limnaeus (Fig. 10 \& Table 1,2). Three differentially expressed genes were shared between the 
14-16 somite escape embryos and newly fertilized escape embryos: Guanine nucleotide exchange factor MSS4, 6-phosphofructo-2-kinase/fructose-2,6biphosphatase, and variations of histone H2A.V. Guanine nucleotide exchange factor MSS4 is downregulated in newly fertilized escape embryos and upregulated in 14-16 somite escape embryos. Six-phosphofructo-2-kinase/fructose-2,6-biphosphatase is upregulated in newly fertilized escape embryos and downregulated in escape embryos at the 14-16 somite stage. Histone $\mathrm{H} 2 \mathrm{~A}$. V is downregulated in escape embryos compared to embryos on a diapause trajectory in both newly fertilized and 14-16 somite embryos. Additionally, two different isoforms of histone acetyltransferase 1, have been identified in 14-16 somite embryos, one highly expressed in escape embryos and the other in embryos bound for diapause II.

In newly-fertilized embryos, a total of 55 genes were identified as being differentially expressed (Table 1,2). Of these genes, 33 (60\%) were upregulated in escape embryos and $22(40 \%)$ in embryos that would enter diapause II. Forty-eight percent of these differentially expressed sequences have significant homology with sequences in the GenBank non-redundant protein database as determined by the Blastx search algorithm.

Twenty-two differentially expressed sequences were identified in 14-16 somite embryos on the two developmental trajectories (Table 3). Eleven of these $(50 \%)$ were identified as upregulated in embryos on the escape trajectory and 11 (50\%) were associated with embryos on the diapause II trajectory. A large proportion 
$(\approx 70 \%)$ of these sequences have significant homology with sequences in the GenBank non-redundant protein database.

\section{Differential Gene Expression in Maternal Tissues}

Sixty-nine sequences were identified as differentially expressed in ovary and liver tissues isolated from female fish producing either high or low proportions of escape embryos (Table $4 \& 5$ ). Of these sequences only one, the membraneassociated progesterone receptor component 1 (ACQ58387.1, Anoplopoma fimbria) shared a consistent expression pattern in both ovary and liver tissues. This gene was $\approx 7$-fold upregulated in both tissues of females producing escape embryos. Liver tissue had two differentially regulated sequences in common with newly fertilized embryos, one has no significant homology to known proteins (ALD37M09) and the other is an integrin-linked protein kinase (NP_956865.1, Danio rerio). These sequences were downregulated in both embryos and tissues associated with an escape embryo trajectory. Beta-tubulin was also differentially expressed in both liver and 14-16 somite embryos. Upregulation of this sequence was associated with escape embryo production in liver tissue (ALD09H02), but it was downregulated in 14-16 somite embryos on the escape trajectory (ALD15L11).

Twenty-three sequences were identified as differentially expressed in maternal ovary with respect to the production of escape or diapausing embryos. Thirteen $(\approx 57 \%)$ of these sequences were upregulated in females producing escape embryos and $10(\approx 43 \%)$ were associated with females producing diapausing embryos (Table 
4). Less than $40 \%$ of the differentially expressed sequences identified from ovary tissue had significant homology with sequences in the GenBank non-redundant protein database.

Maternal liver tissue exhibited 46 differentially expressed sequences in females producing escape embryos relative to those producing diapausing embryos. Twenty-six $(\approx 57 \%)$ of these were upregulated in females producing escape embryos and 20( $243 \%$ ) were downregulated (Table 5). Interestingly, 3 genes were highly expressed in liver tissue isolated from females producing diapausing embryos: one is 40-fold upregulated and has no significant homology to known protein sequences (ALD37P02), the second is a Clasp2 protein that is 25-fold upregulated, and the third is a Chromobox homolog 3 that is induced 24-fold. Of the sequences identified as differentially expressed in liver tissue, $\approx 60 \%$ had significant homology with sequences in the GenBank non-redundant protein database. 

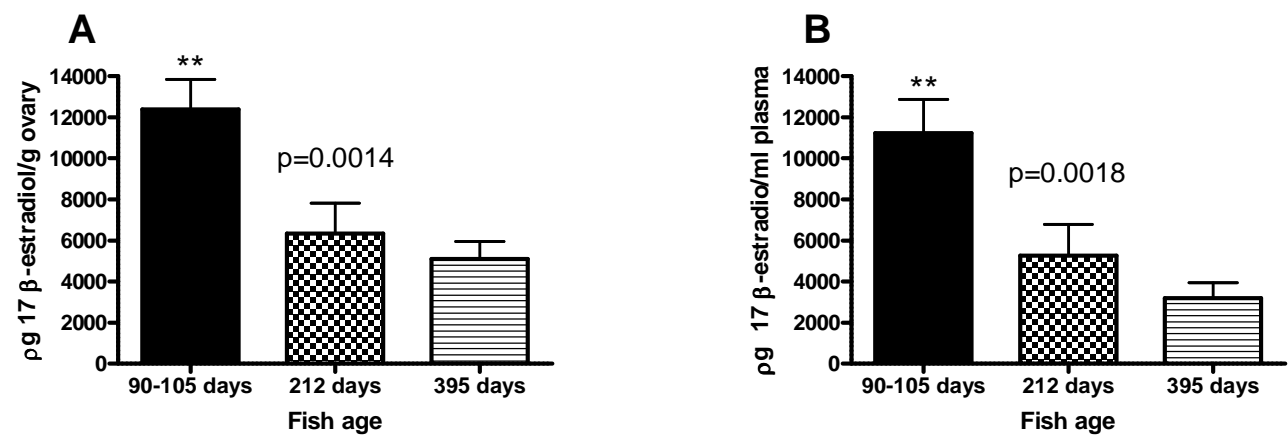

Figure 1. Levels of $17 \beta$-estradiol in (A) ovarian tissue and (B) plasma of female Austrofundulus limnaeus as a function of age. Young females have higher ovarian levels of $17 \beta$-estradiol than older females (ANOVA, $\mathrm{p}=0.0014, \mathrm{~F}=8.54$, Tukey's multiple comparison test: $90-105(n=13)$ vs $212(n=8) p<0.05,90-105$ vs $395(n=8)$ $\mathrm{p}<0.01)$. Plasma $17 \beta$-estradiol levels are also higher in younger than older females (Kruskal-wallis test, $\mathrm{p}=0.0018, \mathrm{KW}$ statistic $=12.69$, Dunn's multiple comparison test: 90-105 (n=12) vs $212(\mathrm{n}=10) \mathrm{p}<0.05,90-105$ vs $395(\mathrm{n}=8) \mathrm{p}<0.01)$. Bars represent mean \pm s.e.m. 


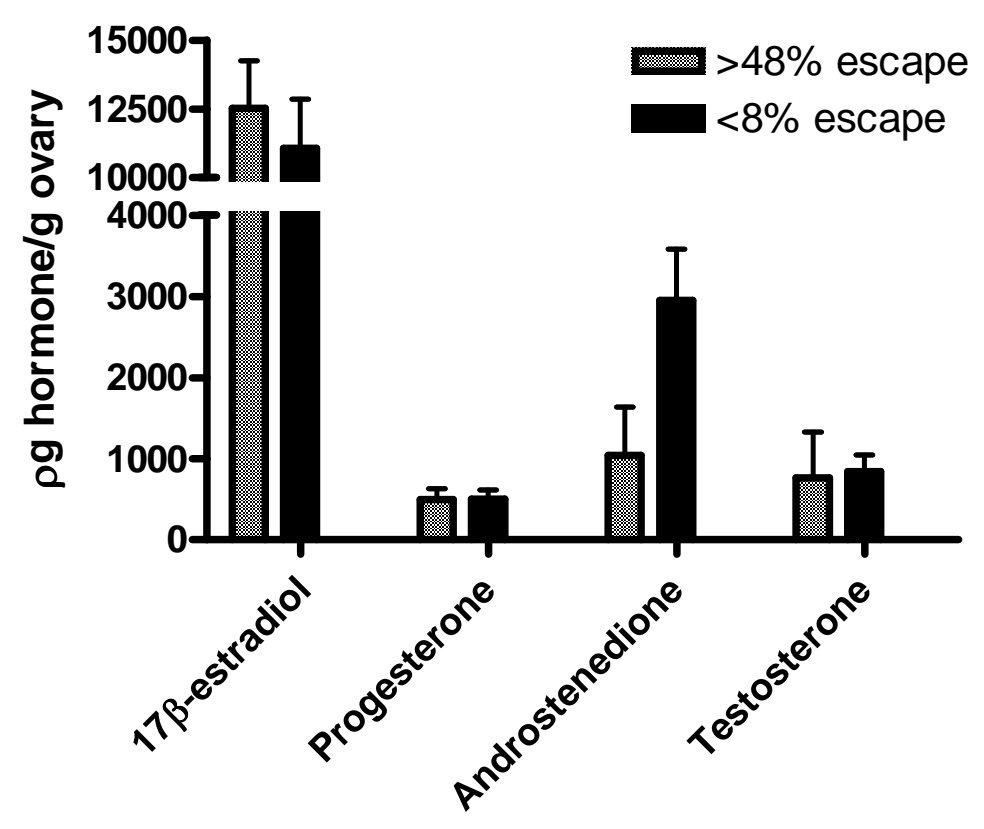

Figure 2. Hormone levels in ovarian tissues isolated from young female A. limnaeus (90-105 days). Black columns are hormone values for fishes producing less than $8 \%$ escape embryos $(n=8)$ and shaded columns $(n=4)$ represent values for females that produced over $48 \%$ escape embryos. The proportion of escape embryos produced was determined over the entire lifetime spawning for that individual fish (total number of spawns ranges from 3-7). There are no statistically significant differences in hormone levels in ovarian tissue isolated from the two groups. Bars represent mean \pm s.e.m. 

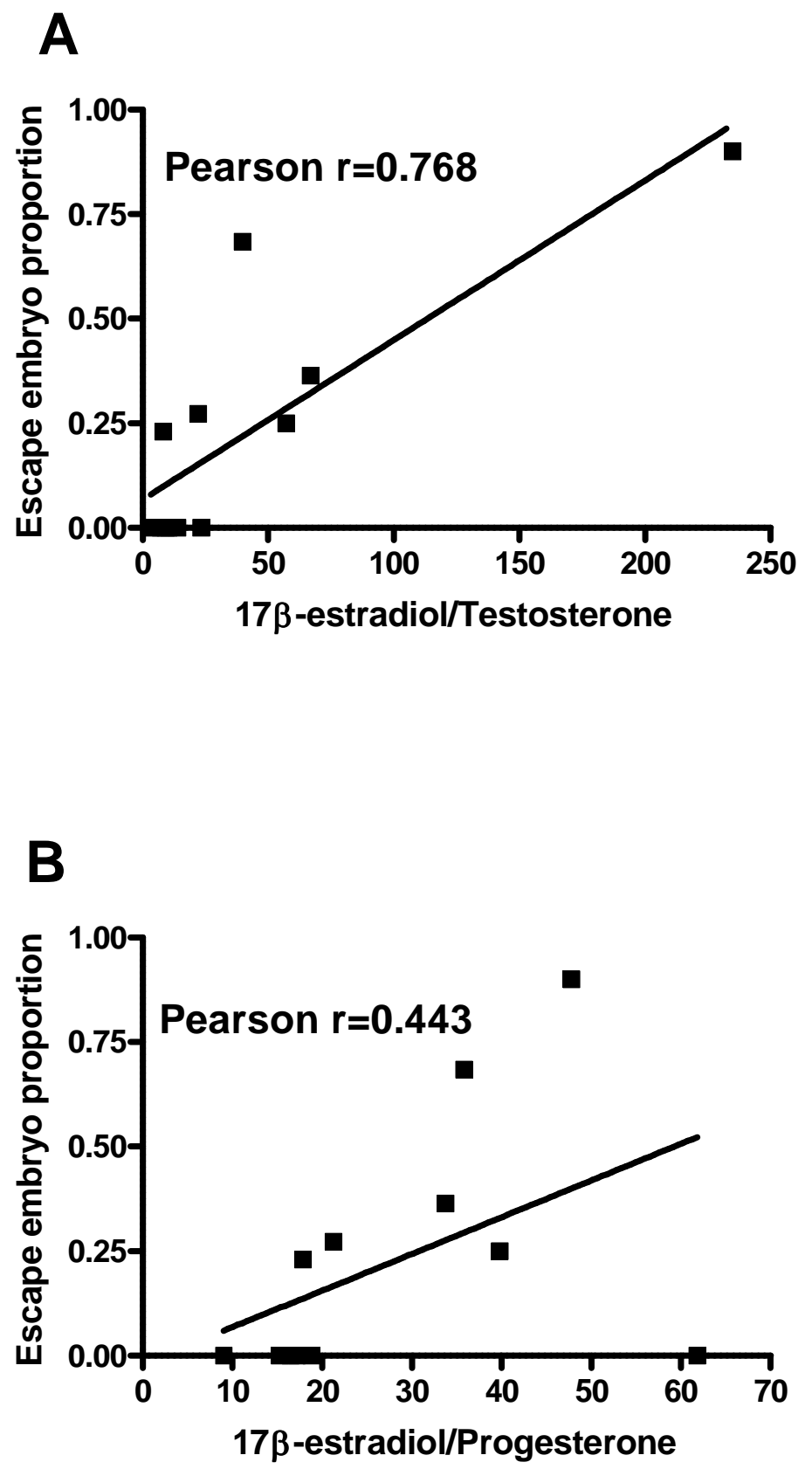

Figure 3. The relationship between the ratio of $17 \beta$-estradiol to (A) testosterone, and (B) progesterone as a function of lifetime escape embryo production in young females (90-105 days). There is a significant correlation between the $17 \beta$ estradiol/testosterone ratio and lifetime production of escape embryos $(\mathrm{p}=0.0035$, Pearson $\mathrm{r}=0.768$ ). 

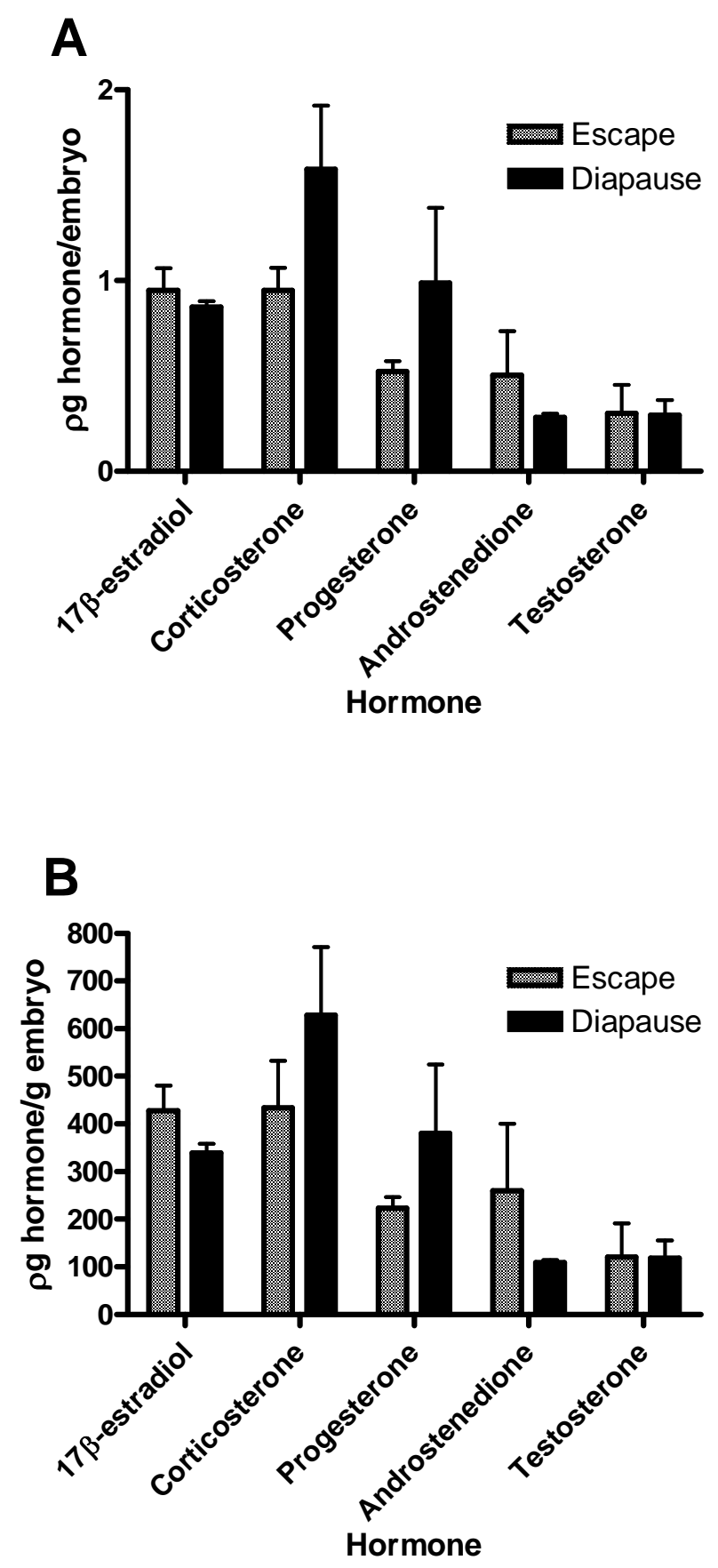

Figure 4. Hormone concentrations in newly fertilized embryos presented (A) per embryo and (B) per g embryo tissue. There are no significant differences between embryos presumed to be on either developmental trajectory. Bars represent mean \pm s.e.m. $(n=4-5)$ 

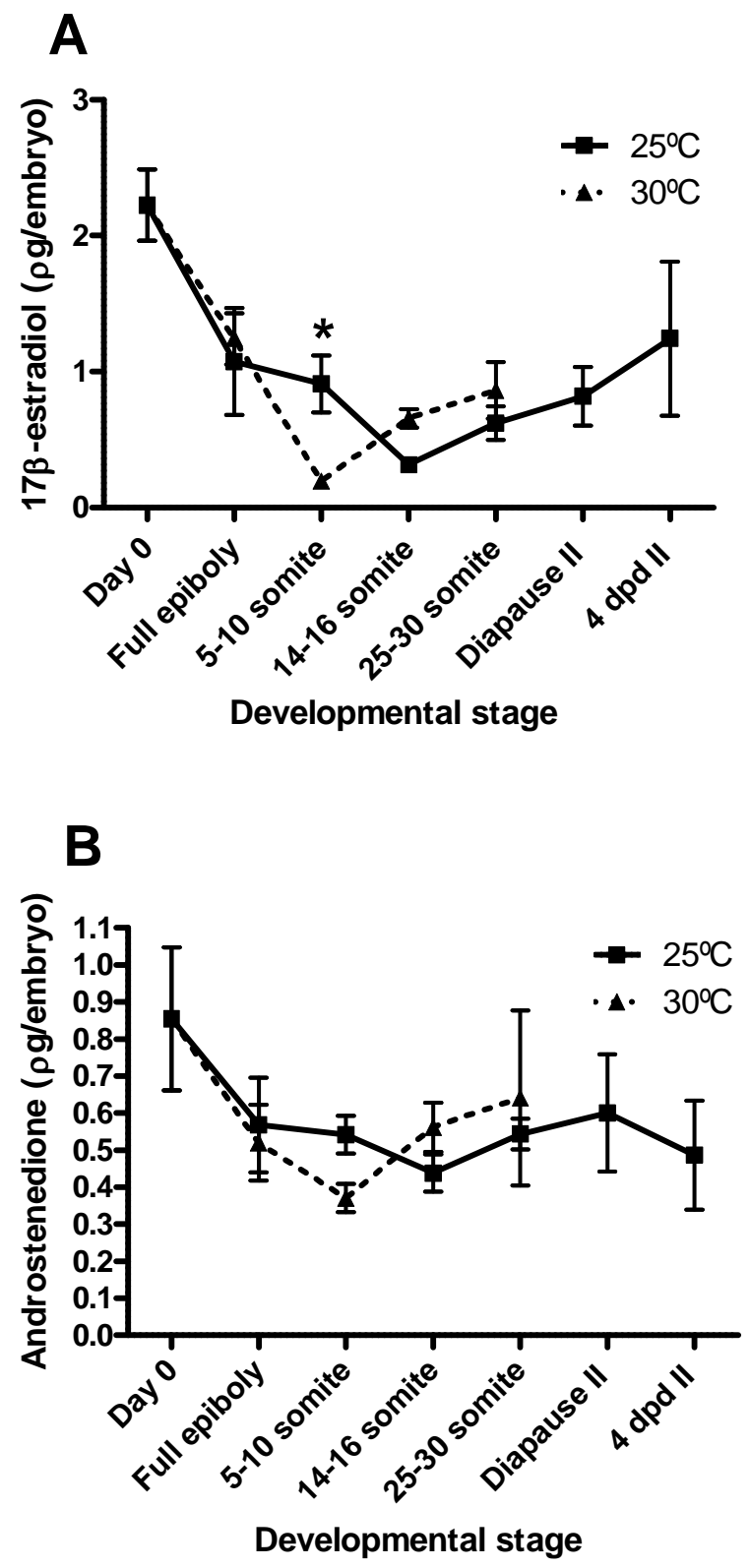

Figure 5. Hormone levels through early development. (A) $17 \beta$-estradiol levels fall through early development, to a significantly lower level in $30^{\circ} \mathrm{C}$ embryos at the $5-10$ somite stage (Two way ANOVA, $\mathrm{p}<0.05, \mathrm{t}=2.925$ ).(B) Androstenedione values follow the same pattern as $17 \beta$-estradiol, although the drop is not significant. Hormone levels increase again following the 5-10 somite stage. This is the developmental period where $30^{\circ} \mathrm{C}$ treatment results in a steep increase in the production of escape embryos. Bars represent mean \pm s.e.m. 

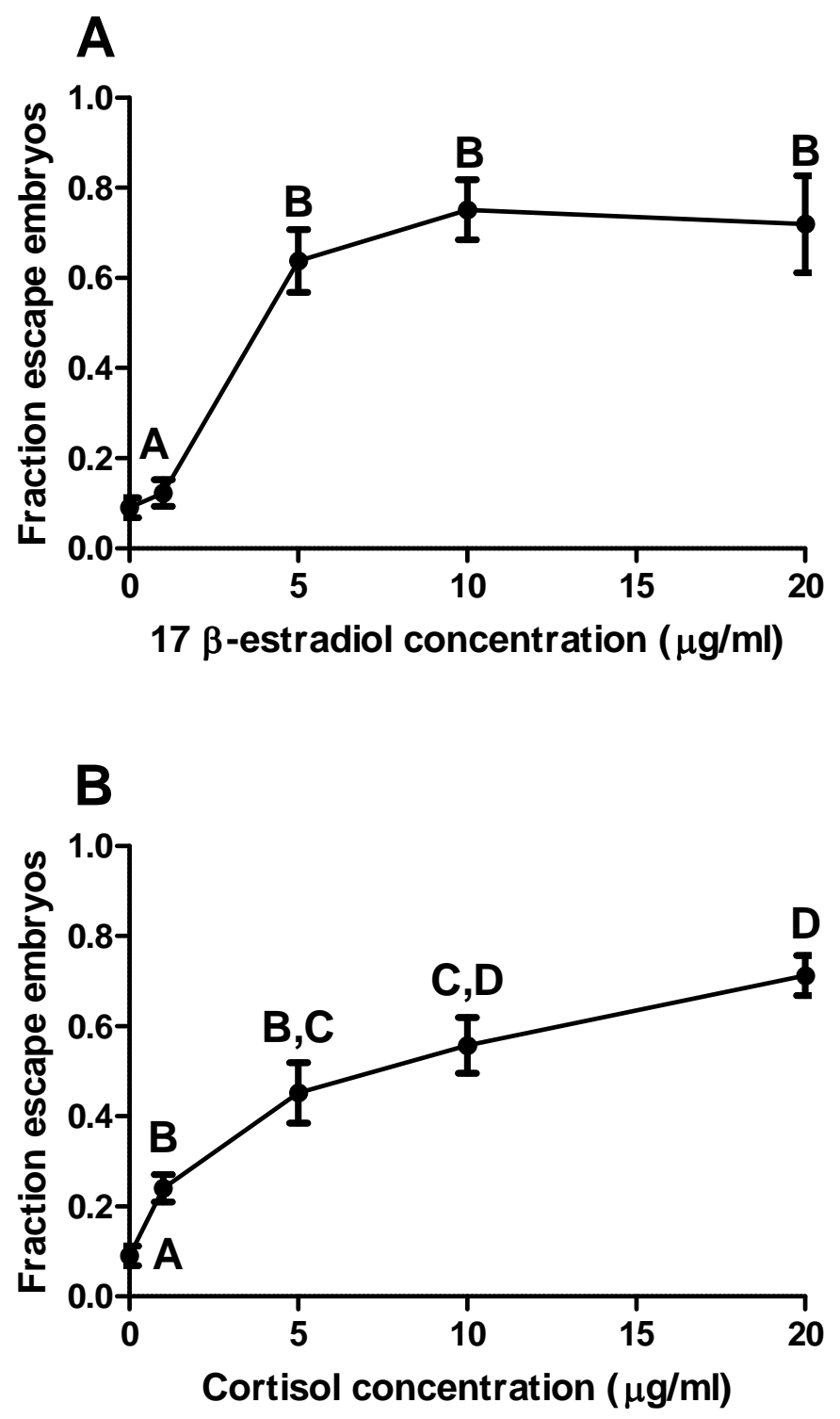

Figure 6. Effect of incubation in exogenous (A) $17 \beta$-estradiol and (B) cortisol on the proportion of embryos that escape diapause II. Exposure to levels of $17 \beta$-estradiol greater than $1 \mu \mathrm{g} / \mathrm{ml}$ results in the majority of embryos following an escape embryo trajectory (Kruskal-Wallis test $\mathrm{p}<0.0001$, KW Statistic $=40.64, \mathrm{n}=12$ ). Cortisol treatment induces an increase in the proportion of escape embryos in a more doseresponse typical fashion (ANOVA $\mathrm{p}<0.0001, \mathrm{~F}=27.39, \mathrm{n}=12$ ). Different numbers above columns represent statistically significant differences. Bars represent mean \pm s.e.m. 


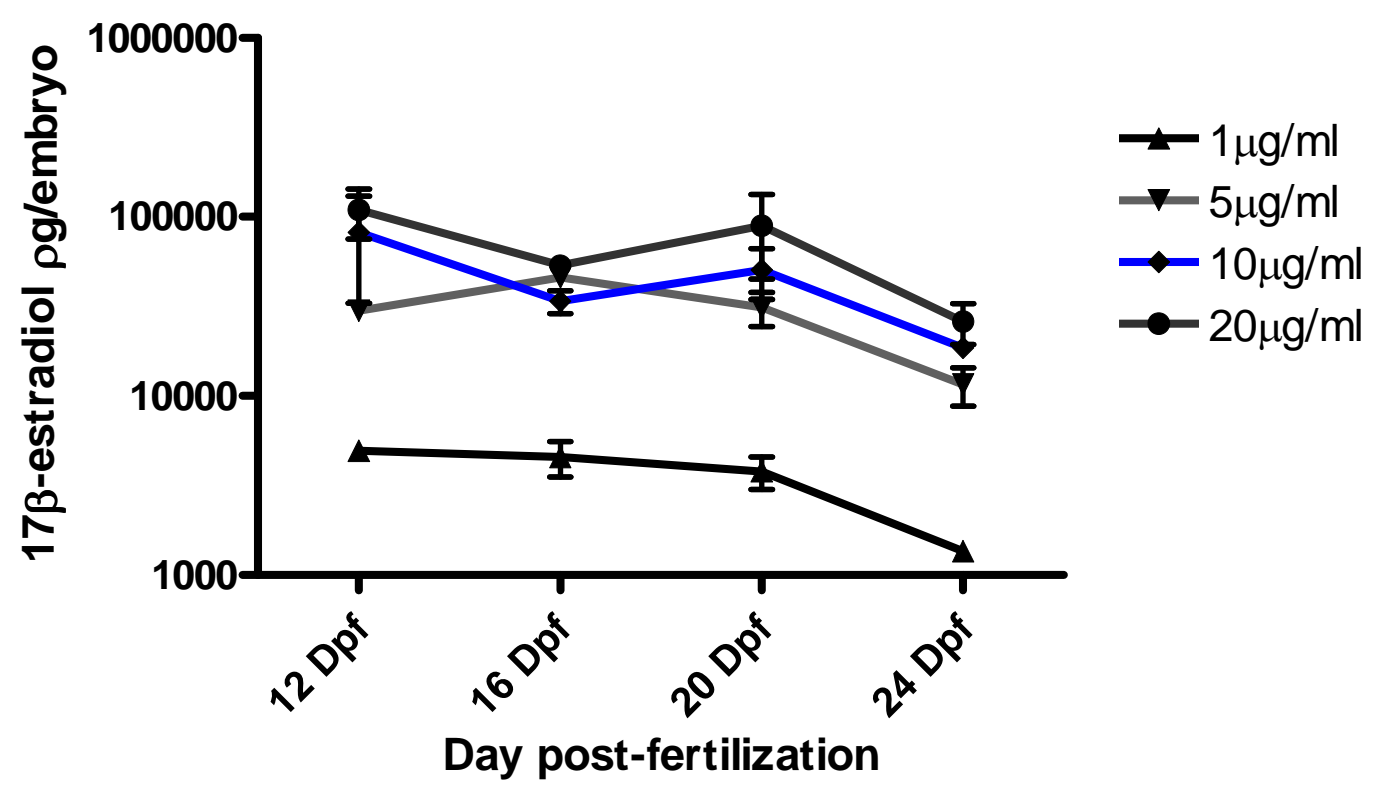

Figure 7. Hormone levels through embryonic hormone treatment; treatment began 6 dpf. Treatment above $1 \mu \mathrm{g} / \mathrm{ml}$ resulted in hormone levels not significantly different at most time points. Bars represent mean \pm s.e.m. 


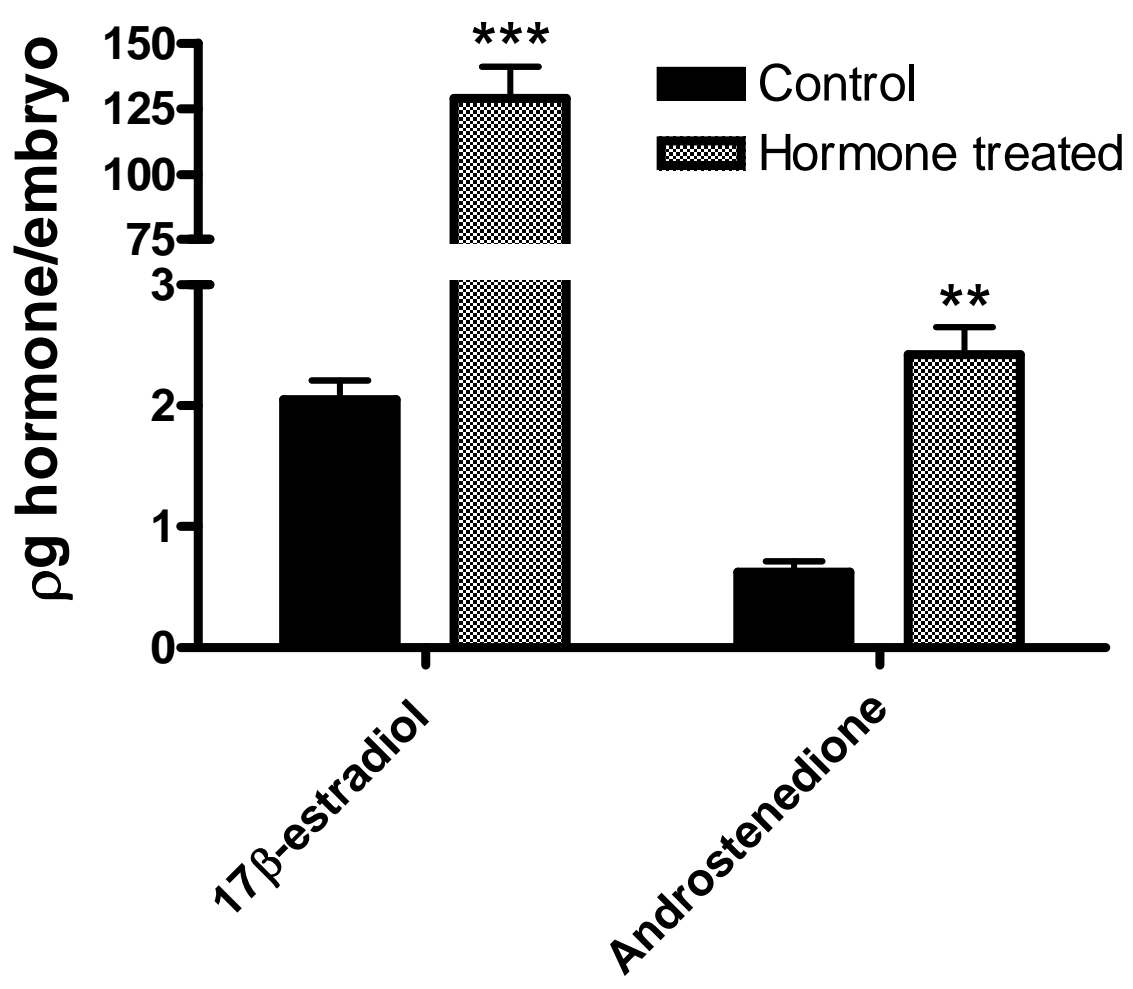

Figure 8. Hormone levels in $17 \beta$-estradiol treated and control dechorionated embryos. Embryos sampled at $18 \mathrm{dpf}$, after 12 days hormone treatment $17 \beta$-estradiol levels are significantly higher in treated embryos $(\mathrm{p}=0.0005, \mathrm{t}=10.48, \mathrm{df}=4)$.

Androstenedione levels in estradiol-treated embryos are significantly higher than in control embryos $(\mathrm{p}=0.0018, \mathrm{t}=7.339, \mathrm{df}=4)$. Bars represent mean \pm s.e.m. 


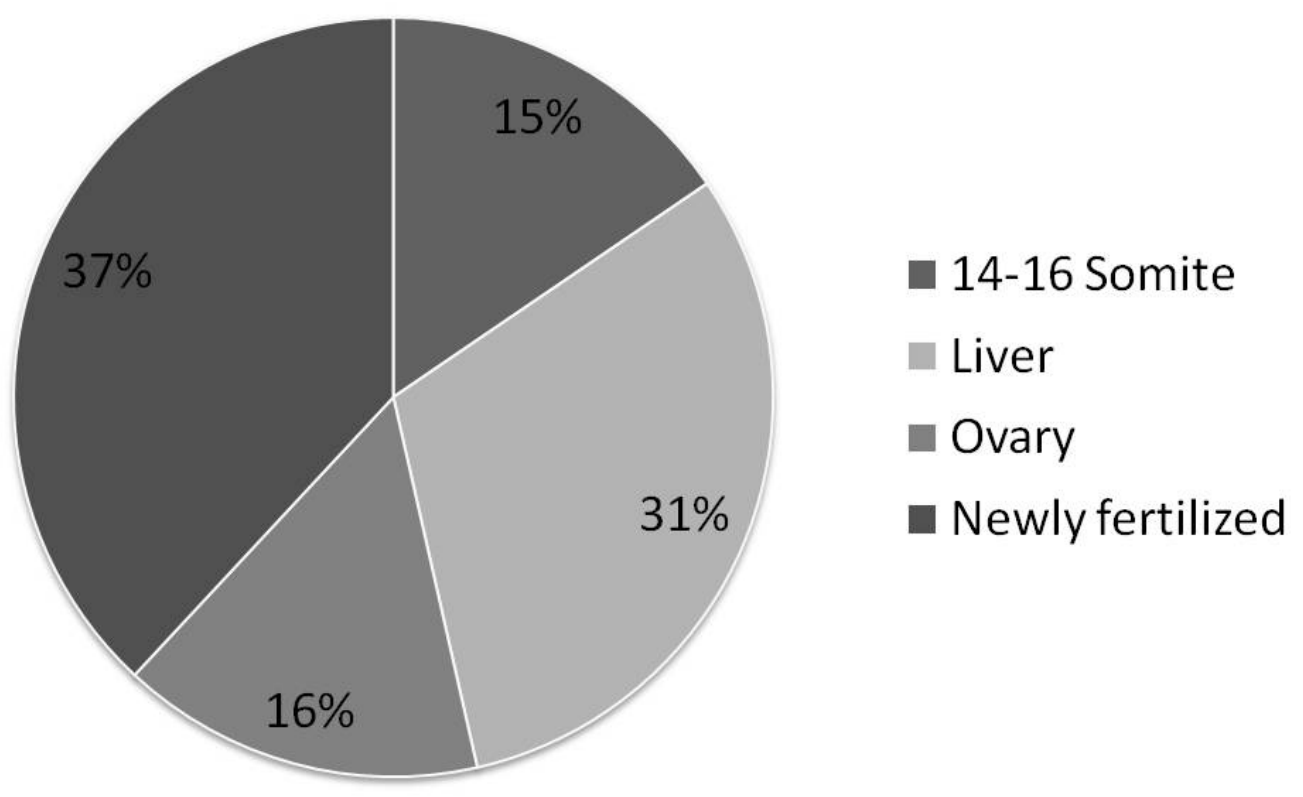

Figure 9. The distribution of sequences found to be differentially expressed in escape embryos and tissues isolated from females producing escape embryos by tissue. Some genes are shared between tissues, therefore total $\neq 100 \%$. 


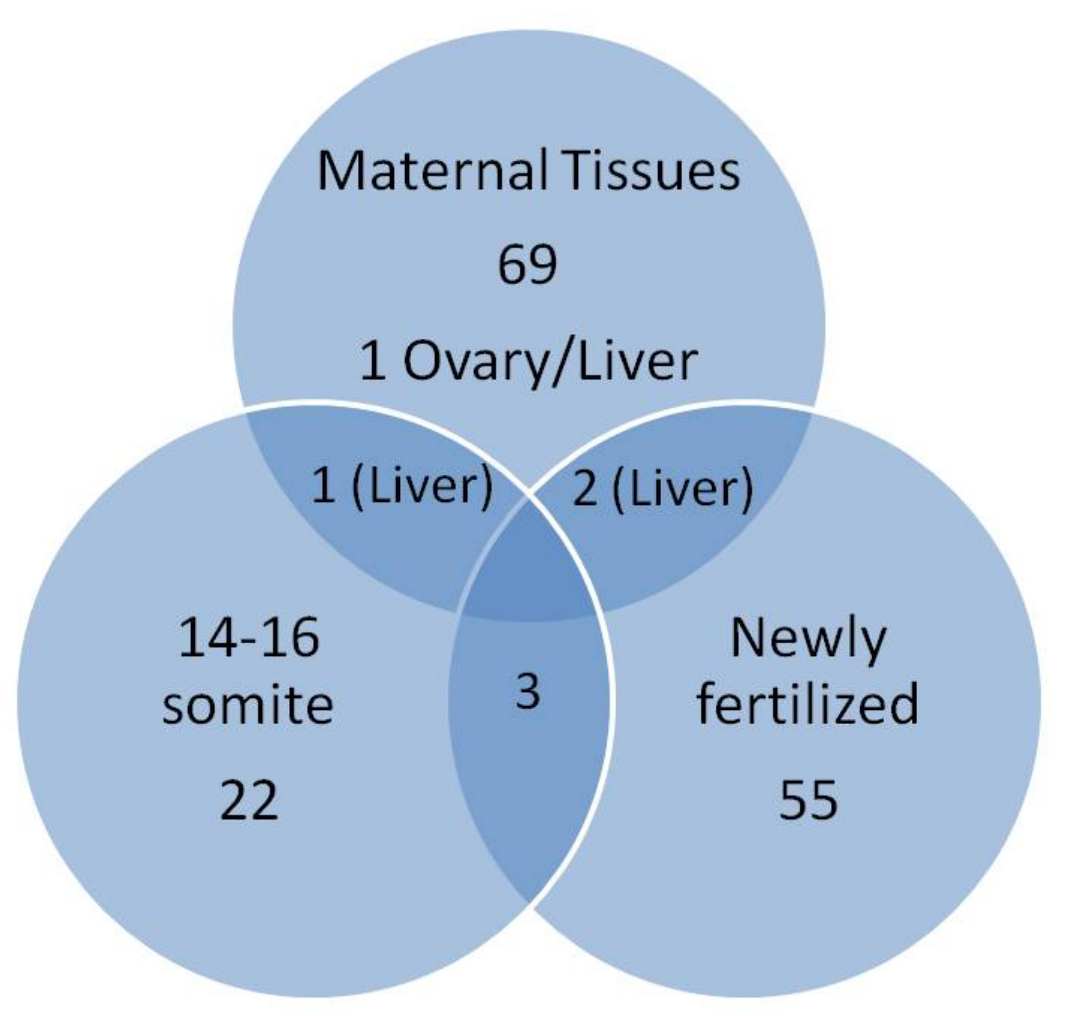

Figure 10. The number of differentially regulated genes identified in each microarray experiment to identify sequences unique to production of escape embryos. Maternal tissues include sequences identified in both ovary and liver tissues. Numbers in overlapping sections represent the number of differentially regulated genes common to both samples. 


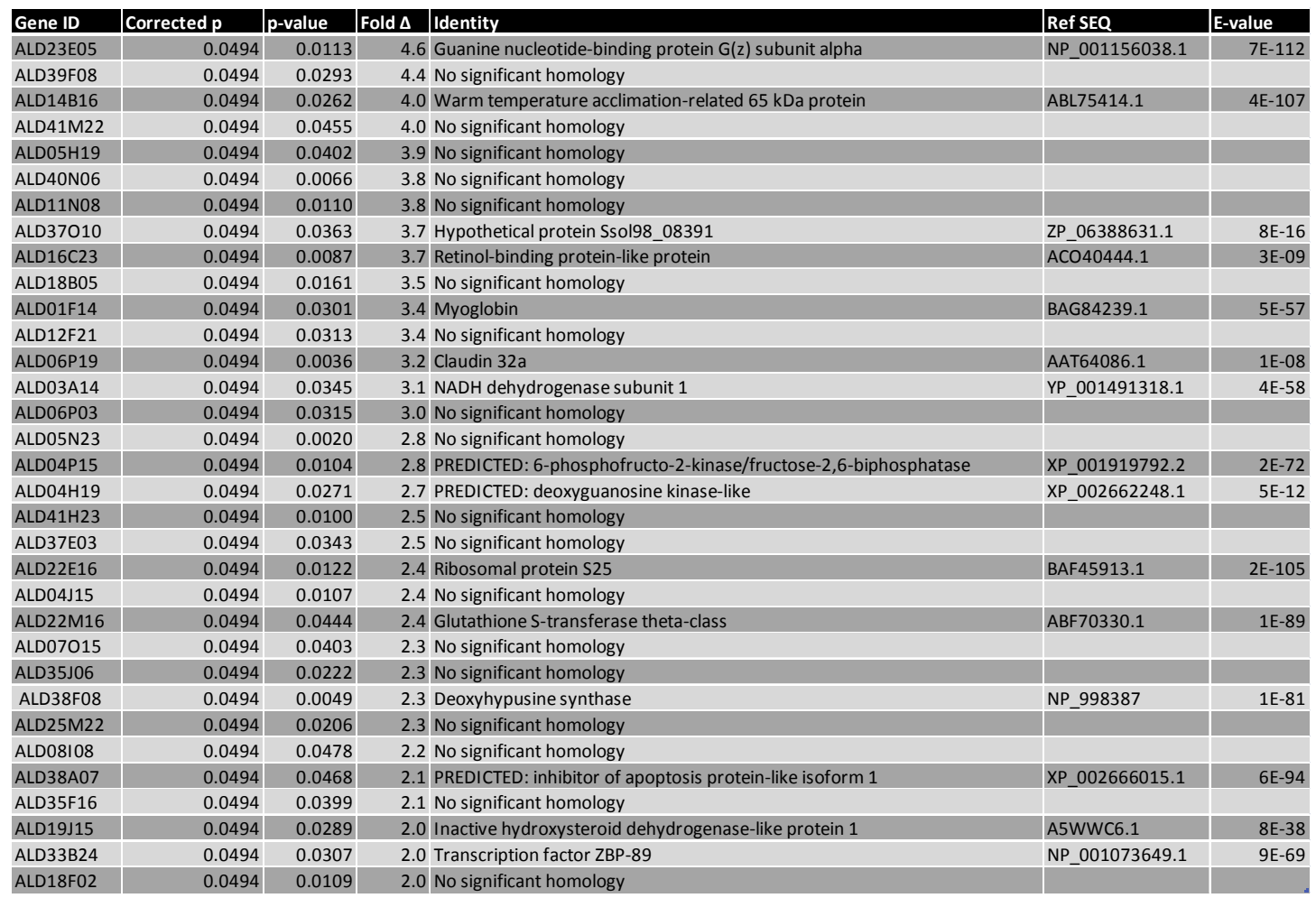

Table 1. Differentially regulated genes in newly fertilized embryos, fold changes are relative to embryos on escape developmental trajectory. Only sequences significantly $(\mathrm{p}<0.05)$ differentially regulated are presented. 


\begin{tabular}{|c|c|c|c|c|c|}
\hline Gene ID & Corrected p & p-value & \begin{tabular}{|l|l|} 
Fold $\Delta$ & Identity \\
\end{tabular} & Ref SEQ & E-value \\
\hline ALD27K10 & 0.0494 & 0.0283 & -3.8 No significant homology & & \\
\hline ALD38K20 & 0.0494 & 0.0247 & -2.6 No significant homology & & \\
\hline ALD33F13 & 0.0494 & 0.0138 & -2.6 Hypothetical protein LOC449817 & NP_001005990.1 & $2 \mathrm{E}-90$ \\
\hline ALD19D01 & 0.0494 & 0.0021 & -2.4 Poly (ADP-ribose) polymerase family, member $12 b$ & NP_001108558.1 & $4 \mathrm{E}-59$ \\
\hline ALD35A12 & 0.0494 & 0.0438 & -2.4 No significant homology & & \\
\hline ALD35I18 & 0.0494 & 0.0341 & -2.4 Eukaryotic translation initiation factor 3 subunit 6 & ACO08955.1 & $8 \mathrm{E}-128$ \\
\hline ALD14G11 & 0.0494 & 0.0131 & -2.3 Serine hydroxymethyltransferase [Danio rerio] & AAX45073.1 & $1 E-118$ \\
\hline ALD42B03 & 0.0494 & 0.0153 & -2.3 Heat shock cognate 71 & ADK88904.1 & $6.00 \mathrm{E}-106$ \\
\hline ALD14K02 & 0.0494 & 0.0394 & -2.3 No significant homology & & \\
\hline ALD38D03 & 0.0494 & 0.0116 & -2.3 No significant homology & & \\
\hline ALD33F04 & 0.0494 & 0.0169 & -2.3 PREDICTED: histone H2A.V-like & XP_002924084.1 & $1 E-54$ \\
\hline ALD24L13 & 0.0494 & 0.0127 & -2.2 Guanine nucleotide exchange factor MSS4 & ACQ58514.1 & $3 E-37$ \\
\hline ALD26J13 & 0.0494 & 0.0284 & -2.2 No significant homology & & \\
\hline ALD28M07 & 0.0494 & 0.0208 & -2.1 Cyclin-dependent kinase 2-interacting protein & NP_001134627.1 & $2 E-11$ \\
\hline ALD35G19 & 0.0494 & 0.0253 & -2.1 No significant homology & & \\
\hline ALD08K10 & 0.0494 & 0.0021 & -2.1 No significant homology & & \\
\hline ALD09K02 & 0.0494 & 0.0127 & -2.1 No significant homology & & \\
\hline ALD26F21 & 0.0494 & 0.0359 & -2.1 Integrin-linked protein kinase & NP_956865.1 & $2 E-73$ \\
\hline ALD33D20 & 0.0494 & 0.0478 & -2.0 Coiled-coil domain-containing protein 111 homolog & NP_001032455.1 & $1 \mathrm{E}-65$ \\
\hline ALD37M09 & 0.0494 & 0.0108 & -2.0 No significant homology & & \\
\hline ALD35120 & 0.0494 & 0.0063 & -2.0 No significant homology & & \\
\hline ALD02J22 & 0.0494 & 0.0272 & -2.0 PREDICTED: cyclin G1-like isoform 2 & XP_002664422.1 & $3 E-87$ \\
\hline
\end{tabular}

Table 2. Differentially regulated genes in newly fertilized embryos, fold changes are relative to embryos on escape developmental trajectory. Only sequences significantly $(p<0.05)$ differentially regulated are presented. 


\begin{tabular}{|c|c|c|c|c|c|c|}
\hline Gene ID & Corrected p & p-value & Fold $\Delta$ & |Identity & Ref SEQ & E-value \\
\hline ALD23D03 & 0.0484 & 0.0354 & 5.1 & CXADR & NP_001124253.1 & $1 \mathrm{E}-72$ \\
\hline ALD16D08 & 0.0467 & 0.0238 & 4.5 & skin mucus lectin & BAI49518.1 & $2.00 E-58$ \\
\hline ALD05E02 & 0.0406 & 0.0024 & 3.0 & No significant homology & & \\
\hline ALD36C23 & 0.0365 & 0.0001 & 2.6 & No significant homology & & \\
\hline ALD40F05 & 0.0419 & 0.0045 & 2.2 & No significant homology & & \\
\hline ALD39L22 & 0.0367 & 0.0003 & 2.1 & Carnosine dipeptidase 1 (metallopeptidase M20 family) & NP_001107394.1 & $1 \mathrm{E}-74$ \\
\hline ALD24L13 & 0.0406 & 0.0037 & 2.1 & Guanine nucleotide exchange factor MSS4 & ACQ58514.1 & $3 E-37$ \\
\hline ALD04P15 & 0.0464 & 0.0223 & -8.7 & 6-phosphofructo-2-kinase/fructose-2,6-biphosphatase & XP_001919792.2 & $2 \mathrm{E}-72$ \\
\hline ALD34H19 & 0.0486 & 0.0400 & -6.0 & Histone H2A.V & XP_002924084.1 & $1 \mathrm{E}-54$ \\
\hline ALD32F04 & 0.0484 & 0.0362 & -5.9 & Histone acetyltransferase type B catalytic subunit & NP_001004572.1 & $4 \mathrm{E}-110$ \\
\hline ALD33D11 & 0.0495 & 0.0472 & -5.6 & No significant homology & & \\
\hline ALD34N22 & 0.0406 & 0.0025 & -4.3 & Flavin containing monooxygenase 5 & NP_001133267.1 & $2 \mathrm{E}-112$ \\
\hline ALD19B23 & 0.0372 & 0.0008 & -4.2 & Alpha-1-antitrypsin & ACJ05607.1 & $2 \mathrm{E}-80$ \\
\hline ALD23I15 & 0.0406 & 0.0021 & -3.5 & Dimethylaniline monooxygenase 5 & NP_001133267.1 & 5E-178. \\
\hline
\end{tabular}

Table 3. Differentially regulated genes in 14-16 somite embryos, fold changes are relative to embryos on escape developmental trajectory. Only sequences significantly $(\mathrm{p}<0.05)$ differentially regulated are presented. 


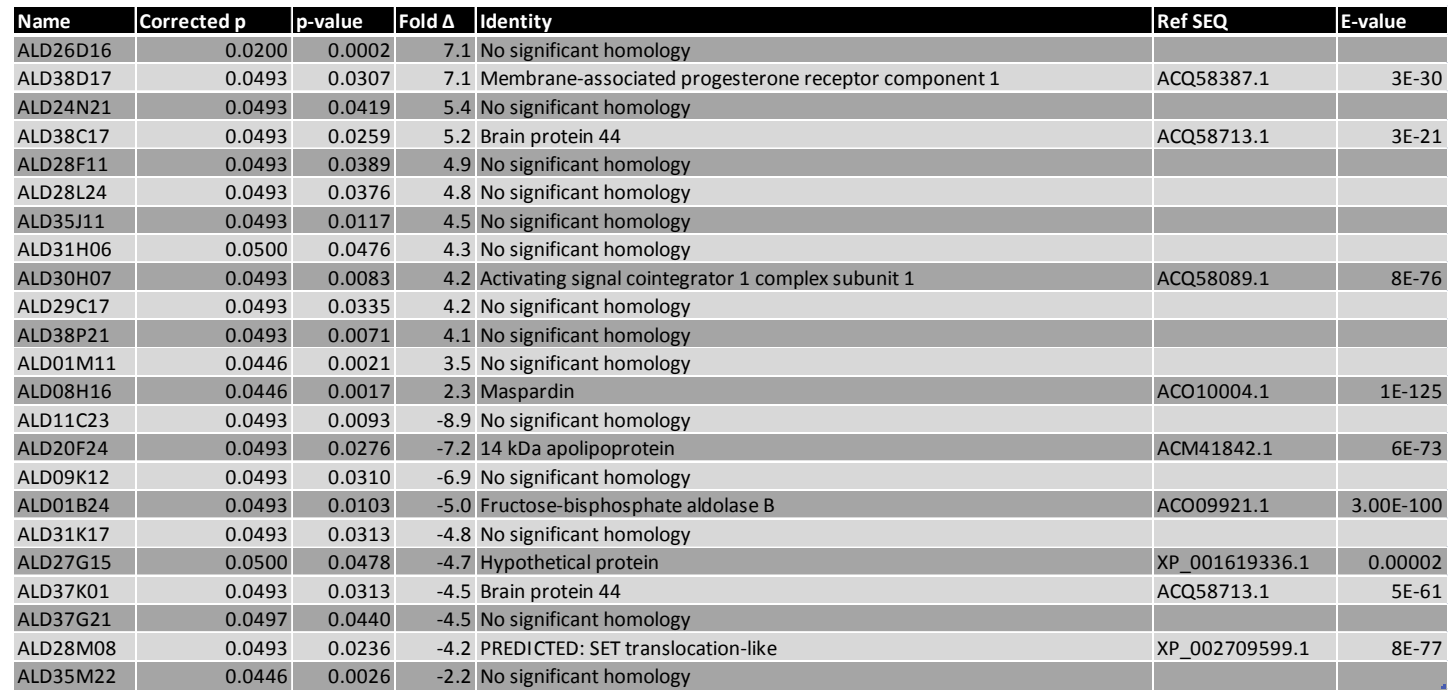

Table 4. Differentially regulated genes in ovary of adult fish. Fold changes are relative to fish producing high proportions of escape embryos. Only sequences significantly $(\mathrm{p}<0.05)$ differentially regulated are presented. 


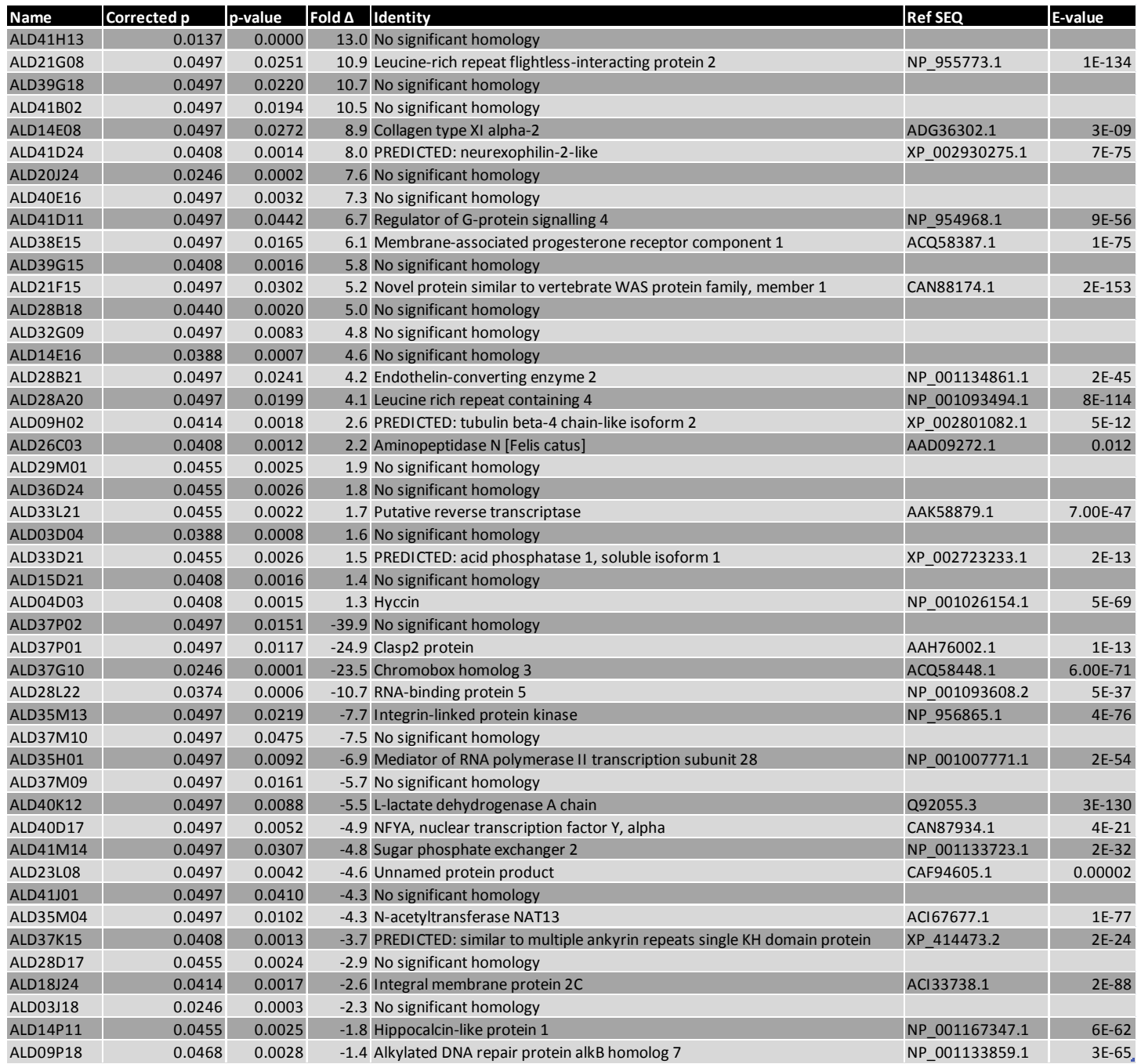

Table 5. Differentially regulated genes in liver of adult fish. Fold changes are relative to fish producing high proportions of escape embryos. Only sequences significantly $(\mathrm{p}<0.05)$ differentially regulated are presented. 


\section{Discussion}

Steroid hormones are powerful modulators of organismal form and function. Entire suites of phenotypic traits, such as sexual dimorphism, can be coordinately expressed through the action of these signaling molecules. This study focuses primarily on the role of estrogens on the regulation of diapause in embryos of the annual killifish A. limnaeus. Estrogens play a major role in regulation of reproduction and metabolism in vertebrates and they are known to have numerous environmentally protective actions. Thus, I hypothesize that estrogen signaling may play a major role in the regulation of metabolic dormancy associated with diapause in embryos of $A$.

\section{limnaeus.}

The effect of estrogens can be mediated through two major routes, genomic and non-genomic. Genomic actions depend on hormones binding to specific receptors initiating a unique gene expression program. Non-genomic actions do not necessarily result in the production of new gene products, but rather cause post-translational alterations in cellular physiology. Non-genomic actions are characterized by rapid initiation (too rapid to be mediated through transcription or translation). In fact, these effects may be realized in cell types lacking the machinery for protein synthesis, and are unchanged by inhibition of nuclear hormone receptors or the machinery of translation (Falkenstein et al., 2000; Revelli et al., 1998). Interestingly, the effects are not blocked by hormone adhesion to elements too large to pass through the cell 
membrane, and thus are likely mediated by membrane-bound receptors. Non-genomic actions of estrogens are specific, and similar but non-identical hormone molecules can exert different magnitudes of response (Persky et al., 2000; Falkenstein et al., 2000; Revelli et al., 1998). These non-canonical actions of estrogens have been shown to initiate physiological changes that can protect heart muscle and skeletal elements from oxidative damage (Persky et al., 2000), and estrogen has also been shown to protect against ischemic damage (Dubal et al., 1998). Evidence presented below suggests estrogen may be acting via both genomic and non-genomic routes to regulate the fate of A. limnaeus to either enter or escape diapause II.

\section{Maternal Effects on Developmental Trajectory}

Overall, the steroid hormone levels reported here for female A. limnaeus correspond well to published values for other killifish species (Fundulus heteroclitus plasma; $\approx 10,000-30,000$ pg/ml $17 \beta$-estradiol; Kryptolebias marmoratus plasma hermaphrodite $\approx 3,000 ;$ male $\approx 7,000 \rho \mathrm{g} / \mathrm{ml} 17 \beta$-estradiol), however estrogen levels are elevated in comparison to other reported values for teleosts (Minamimoto et al., 2005; Cochran et al., 1988; Webb et al., 2002; Wang et al., 2008; Sisneros et al., 2004; Bradford and Taylor, 1987; Rahman et al., 2000; Hobby and Pankhurst, 1997; Feist et al., 1990; Bayunova et al., 2003; Barannikova et al., 2003). $17 \beta$-estradiol is higher in female A. limnaeus plasma (range 292-26,532 $\mathrm{\rho g} / \mathrm{ml}$ ) when compared to values reported for other non-killifish teleost species (Poricthys notatus undetectable-13,888 


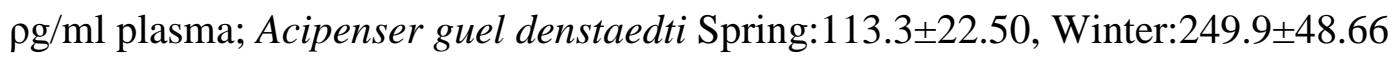
$\rho g / \mathrm{ml}$ plasma). This may be a function of annual killifish continually spawning, whereas other reported values are for fish spawning seasonally or on a more intermittent basis (Cochran et al., 1988; Wang et al., 2008; Sisneros et al., 2004; Bradford and Taylor, 1987). Conversely, testosterone levels are much lower than estrogen in female A. limnaeus (814 $\mathrm{gg} / \mathrm{g} \pm 217$, range $65-2,460 \rho \mathrm{g} / \mathrm{g}$ ovary tissue), and also lower than testosterone values reported for some other species (Fundulus heteroclitus $620 \pm 330-8,090 \pm 2,750$ pg/ml plasma; Poricthys notatus undetectable-

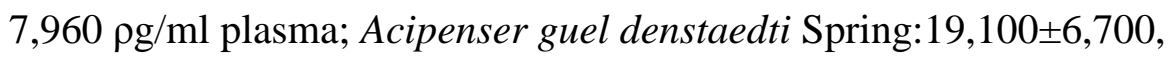
Winter: $18,700 \pm 3,320 \rho \mathrm{g} / \mathrm{ml}$ plasma), although these values are reported in plasma (Cochran et al., 1988; Hobby and Pankhurst, 1996; Bayunova et al., 2003). The elevated estrogen levels and relatively low testosterone levels observed may be explained by higher aromatase activity in A. limnaeus ovary tissue, but no data is currently available on aromatase activity in this species.

Levels of $17 \beta$-estradiol are known to decrease in female A. limnaeus as they age (Fig. 1), and this trend is correlated with a decrease in the production of escape embryos in older females (Podrabsky et al., 2010). This observation led me to hypothesize that differences in the maternal levels of $17 \beta$-estradiol may affect the developmental trajectory of embryos. However, I found no direct correlation between estrogen levels in maternal ovary tissue and the production of escape embryos (Fig. 2). While overall levels of estrogen are not correlated with escape embryo production, the ratio of estrogen to testosterone is significantly correlated with the production of 
escape embryos (Fig. 3, r= 0.768) and suggests that steroid hormone metabolism may indeed still be linked with the production of escape embryos. This correlation suggests a role for cytochrome $\mathrm{P} 450$ aromatases in determining developmental pathway; greater action by aromatases would result in this higher ratios and thus higher production of escape embryos. In teleosts, two aromatase proteins with similar catalytic activity are responsible for the production of estrogens through the aromatization of androgens (Callard et al., 2002; Piferrer and Blazquez, 2005). Interestingly, the membrane associated progesterone receptor component 1 (PGRMC1), that binds and positively regulates cytochrome P450 proteins (Hughes et al., 2007), is upregulated in ovary and liver of fish producing escape embryos (Table $4 \& 5)$. PGRMC1 is known to be expressed in the cytoplasm but has also been shown to localize with cellular membranes in ovary tissue. The protein transduces the antiapoptotic action of progesterone in the ovary (Peluso, 2006; Peluso et al., 2008), although it does not directly bind progesterone (Min et al., 2005). The protein is thought to be involved in steroidogenesis (Zhang et al., 2008) and PGRMC1 interacts with multiple cytochrome P450's involved in progesterone and estrogen synthesis (Rohe et al., 2009). Inactivation of PGRMC1 can decrease the actions of cytochrome P450's, blocking cholesterol synthesis (Hughes et al., 2007), possibly through its interactions with a regulator of cholesterol homeostasis, Insig-1 (Suchanek et al., 2005). This action results in an increase in toxic sterol intermediates, and also interferes with pharmaceutical metabolism (Hughes et al., 2007). Thus, the 
upregulation of PGRMC1 could change the hormonal environment of the ovary in females producing escape embryos through alterations in ovarian steroid metabolism.

Other evidence suggests a role for altered ovarian hormone metabolism with the production of escape embryos. The transcript for a $14 \mathrm{kDa}$ apoplipoprotein involved in cholesterol transport and binding is downregulated in ovary tissue isolated from females producing escape embryos (Table 4). The activity of the closely related apolipoprotein A1 is enhanced by estradiol, and decreased by androgens (Hargrove et al., 1999), providing another link between hormonal levels and altered steroid metabolism. In chicken granulosa cells (Morley et al., 1992), human oocytes (Tesarik and mendoza, 1995), and rat endometrial tissue (Pietras and Szego, 1975; Pietras and Szego, 1977), treatment with $17 \beta$-estradiol resulted in rapid uptake of $\mathrm{Ca}^{2+}$ (Revelli et al., 1998), which has been shown to inhibit aromatase activity (Balthazart et al., 2001; Balthazart et al., 2003). Importantly, this estrogen mediated increase in $\mathrm{Ca}^{2+}$ has been demonstrated on unfertilized oocytes (Tesarik and Mendoza, 1995), providing clear evidence that maternal hormone concentrations can immediately affect the intracellular milieu of developing oocytes. Aromatase expression has been demonstrated in embryos of Danio rerio, at different levels during development (Lassiter et al., 2002), and thus embryos of A. limnaeus very likely have the capacity to respond to steroid hormones through alterations in cytochrome P450 activity. Future studies should focus on the role of aromatase activity and steroid hormone metabolism in maternal tissues and their relationship to the production of escape embryos. 
$17 \beta$-estradiol has also been shown to exert affects on other growth related molecular pathways via non-genomic pathways. The mitogen activated protein kinase (MAPK) pathway was induced by treatment with conjugated membrane impermeable $17 \beta$-estradiol, through the phosphorylation of ERK-1 and ERK-2 (Watters et al., 1997). This pathway governs the duration of diapause in the silkworm (Fujiwara et al., 2006; Iwata et al., 2005) and the termination of embryonic diapause in a fleshfly (Fujiwara and Denlinger, 2007). Through these non-genomic actions, higher maternal estrogen levels could constitute the basis of profound effects on developing embryos, through modification of transcript deposition and phosphorylation state of maternally derived proteins.

In any hormone system, signaling can only be accomplished by the presence of active hormone and an active cellular receptor. The hormonal environment of unfertilized eggs has profound influences on the quality of the developing fish (Brooks et al., 1997; McCormick, 1998). Thus, the effects of estrogen on the regulation of diapause in A. limnaeus could be mediated through the expression of estrogen receptor variants. In teleosts, three different estrogen receptors have been identified, showing differences in tissue expression and differences in estrogenic stimulation based on tissue (Bardet et al., 2002; Greytak and Callard, 2007; Hawkins et al., 2000; Menuet et al., 2001; Callard et al., 2001; Menuet et al., 2002). Estrogen receptor activity can also be rapidly modified through phosphorylation (Balthazart et al., 2001). Nothing is currently known about estrogen receptor expression, activity, or regulation in relation to production of escape embryos in A. limnaeus. This an 
obvious area for future research to focus based on the interesting effects of estrogen on the production of escape embryos, but the lack of a clear relationship between levels of estrogen and escape embryo production.

The upregulation of the transcript for a nuclear-receptor coactivator, activating signal cointegrator-1 (ASC-1), in ovary tissue from females producing escape embryos suggests an intriguing link between nutritional environment, steroid hormones, and the production of escape embryos. This protein acts as a transcriptional coactivator of nuclear receptors, including estrogen receptors and the steroid receptor coactivator, SRC-1 (Kim et al., 1999; Lee et al., 2001). ASC-1 binds target nuclear receptors or transcription factors, and additionally binds the RNA polymerase 2 complex, modifying transcriptional dynamics (Kim et al., 1999). In addition to enhancing the effects of steroid hormones, this family of coactivators also possesses histone acetyltransferase activity and is thus likely involved in mediating chromatin remodeling directed by steroid hormones (Xu and O'Malley, 2002). Rapid changes are found in the subcellular localization of ASC-1 under different nutritional states. ASC-1 is normally located in the nucleus, however after 24 hours of serum deprivation it is largely translocated to the cytoplasm. This change in localization is reversed when SRC-1 or its other coactivators are present, or under serum supplementation (Kim et al., 1999; Lee et al., 2001). ASC-1 could act as a link between nutritional status, endogenous hormonal cues, and changes in the ovary that alter the developmental trajectory of embryos produced. Annual killifish embryos can also respond to the presence of adults by entering developmental arrest at diapause I 
or II (Inglima et al., 1981; Levels, 1986; Levels, 1988). It is tempting to suggest that this signal or a similar one, could function to alter the developmental trajectory of embryos as a function of nutritional status.

The effect of maternal nutritional status on the production of escape embryos has not been investigated, but this is a very promising and ecologically relevant cue; poor nutritional status of the female would likely cue for an unsuitable environment for hatching and growth of offspring. Nutritional status is a known to affect entrance into developmental dormancy in a number of other systems. C. elegans larvae enter the dauer developmental arrest in response to dauer hormone, produced by nearby juveniles and adults under poor nutritional conditions (Butcher et al., 2007; Tatar et al., 2003; Gerisch and Antebi, 2004; Patel et al., 2008). Dormancy in Daphnia has also been shown to respond to maternal nutritional status. A poor maternal nutritional environment can result in a switch from parthenogenetic to bisexual reproduction and the production of ephippia, two dormant offspring attached to the back of the mother (LaMontagne and McCauley, 2001; Alekseev and Lampert, 2001; Alekseev and Lampert, 2004). This effect can be transmitted through multiple generations, and is also modified by environmental cues such as photoperiod and predator abundance (Alekseev and Lampert, 2001; Alekseev and Lampert, 2004). There are many similarities between dormancy in these invertebrate systems and in regulation of diapause in annual killifish embryos. The results of the present study suggest that maternal nutritional status may be one way that the production of diapausing embryos 
is regulated. Future studies on the role of maternal nutritional status and the putative role of ASC-1 in mediating this signal will likely shed light on this possibility.

The potential role of epigenetic changes in maternal determination of developmental pathway, suggested through the interaction of ASC-1 with SRC-1, is strengthened by differential expression of other sequences identified. The SETtranslocation like gene is upregulated in ovary from females producing diapausing embryos. SET is involved in gene silencing through the inhibition of demethylation. Specifically, it binds to targets of histone acetyltransferases (Jung et al., 2002). Chromobox homologue $3(\mathrm{Cbx} 3)$, also known as heterochromatin protein $1 \gamma$, is 25 fold upregulated in liver of females producing diapausing embryos compared to those producing escape embryos. The most common function of this protein is the production of heterochromatin through histone binding, typically resulting in gene silencing (Lomberk et al., 2006). Additionally, members of the Cbx family are known to interact with non-histone proteins involved in transcriptional regulation, DNA repair, and chromatin modification (Lomberk et al., 2006). These factors could induce epigenetic changes, resulting in relatively rapid changes in proportions of embryos on alternate developmental pathways, as seen in A. limnaeus.

\section{Embryonic Hormonal Environment and Developmental Trajectory}

Exposure to high levels of both estrogen and cortisol can cause embryos of $A$. limnaeus to shift from a diapausing to an escape developmental trajectory (Fig. 6). 
Quantification of estrogen levels in embryos treated with exogenous $17 \beta$-estradiol indicate similar levels of hormone accumulated in embryos that is independent of hormone concentrations exceeding $1 \mu \mathrm{g} / \mathrm{ml}$ concentration (Fig. 7). The levels of hormone delivered to whole embryos in this manner suggests a massive dosage of hormones. However, when estrogen levels were measured in embryos first exposed to $5 \mu \mathrm{g} / \mathrm{ml}$ estrogen and then dechorionated prior to quantification of hormone levels, the concentration of hormones in the cellular and yolk compartments of the embryos are several orders of magnitude lower (Fig. 8). Thus, greater than $99 \%$ of the hormone is absorbed to the chorion, and is not likely active within the embryo, which results in exogenous exposures that are about 100 times those found in control embryos (Fig. 8). Importantly, $17 \beta$-estradiol treatment in embryos also resulted in significantly elevated levels of androstenedione (Fig. 8), a precursor of both testosterone and estrogen biosynthesis. This provides circumstantial evidence of aromatase activity modifying embryonic hormone levels with high levels of estrogen. This effect could result from aromatase activity in reverse, converting exogenous estrogens into androgens, or by high estrogen levels inhibiting aromatase as has been reported in another system (Shimizu et al., 2003), that could result in an increase in biosynthetic precursors such as androstenedione from normal metabolism.

Traditionally, lipophilic hormone levels in embryos were thought to be regulated by hormone levels in the maternal environment, with passive transfer from maternal plasma and the cytosol of theca and granulosa cells into developing offspring (Schwabl, 1993; Moore and Johnston, 2008). This passive model of 
hormone transfer would result in higher levels of hormones in the yolk than maternal plasma, because of the lipophilic status of steroid hormones and the lipid nature of yolk (Moore and Johnston, 2008). However, newly fertilized embryos of A. limnaeus exhibit hormone levels that are considerably lower than those in the ovarian tissue from which they are derived (Fig.2, 4B). This suggests active regulation of hormone partitioning into embryos. Some mechanisms for regulation of hormone transfer have been proposed including an enzymatic barrier (Moore and Johnston, 2008) and a barrier to diffusion (Licht et al., 1998). Localized steroid production could also create an ovarian hormone gradient, although this is unlikely in light of our data showing the large differences between ovary and embryo hormone levels. At present the mechanism resulting in this difference between newly fertilized embryos and the ovary environment is unclear.

Embryos developing along the two different developmental trajectories appear to have different rates of estrogen metabolism (Fig. 5). Embryos incubated at $30^{\circ} \mathrm{C}$ (which will escape diapause II) metabolize both estrogen and androstenedione at a faster rate than embryos developing at $25^{\circ} \mathrm{C}$ that will enter diapause II. These differential rates of degradation result in significantly lower concentrations of these hormones in the 5-10 somite embryo. This stage also appears to be the developmental time that embryonic estrogen synthesis is initiated in escape embryos, while in embryos entering diapause it is not initiated until the 14-16 somite stage. There has been surprisingly little research on embryonic hormone production in teleosts. Some earlier studies have indicated little capacity for hormone synthesis in teleost embryos 
prior to full yolk absorption (Brooks et al., 1997). Other results indicate an initial decline in steroid hormone levels, followed by a moderate increase (De Jesus et al., 1992). In Coho salmon embryos, steroid hormones decreased through development until hatching (Feist et al., 1990). Estradiol levels also decreased through development in snapping turtle embryos (Elf et al., 2002). Our results indicate that in A. limnaeus, hormone synthesis is initiated during the early stages of somitogenesis, and at different developmental stages for the two developmental trajectories. This period overlaps with the developmental window when incubation at $30^{\circ} \mathrm{C}$ results in a developmental switch from a diapausing to an escape embryo trajectory (Podrabsky et al., 2010). Thus, differences in hormone metabolism are correlated with developmental trajectory in this species, although a causative link has yet to be identified.

There is no direct relationship between hormone concentrations in newly fertilized embryos that will follow a diapausing or escape embryo trajectory (Fig. 4). The fact that high levels of estrogen can affect developmental trajectory, and the evidence presented above for altered gene expression in maternal tissues suggests that the effects of hormones on developmental trajectory could be mediated through alteration of hormone receptor expression or activity. Altered activity of steroid receptors in embryos could easily result in different sensitivity to the same total quantity of hormones. Increasing the hormone levels during oogenesis may alter hormone receptor levels or program for a different expression program for these receptors during early development. Steroid hormone receptor mRNA's are known to 
be deposited into zebrafish embryos (Pikulkaew et al., 2009). Although our microarray studies did not identify differential levels of estrogen receptors in embryos, a transcript for a hydroxysteroid dehydrogenase (HSD) enzyme is upregulated in embryos destined to become escape embryos compared to those on a diapausing developmental trajectory (Table 1). HSD's have generated much interest due to their roles in hormone metabolism and disease (Moeller and Adamski, 2006; Tomlinson et al., 2004). In C. elegans, HSD1 null mutants initiate the dauer diapause stage inappropriately, and are hypersensitive to dauer hormone (Patel et al., 2008). The HSD identified in this study has the highest homology to a catalytically inactive form, HSDL1. This protein has a point mutation in the active center, that renders it enzymatically inactive in humans and zebrafish, and that is conserved in many other vertebrates (Meier et al., 2009). However, the widely conserved status of this protein, together with identified protein interactions in yeast, suggest a regulatory role for HSDL1 (Meier et al., 2009).

As in adult ovary tissue, epigenetic changes seem to play a role in the switch between escape and diapause developmental pathways within the embryo. One of the genes that is diferentially expressed in newly fertilized escape embryos, and 14-16 somite stage escape embryos induced by incubation at $30^{\circ} \mathrm{C}$ is histone $\mathrm{H} 2 \mathrm{Av}$. This transcript is downregulated at both developmental stages in association with the escape embryo trajectory. Histone $\mathrm{H} 2 \mathrm{Av}$ is a member of the $\mathrm{H} 2 \mathrm{Az}$ variant histone family; members of this family are involved in transcriptional activation and repression (Dhillon and Kamakaka, 2000). In Drosophila, H2Av is involved in 
heterochromatin formation and gene silencing (Swaminathan et al., 2005), and in yeast seems to flank euchromatin regions, inhibiting the spread of heterochromatin (Kobor et al., 2004). The downregulation of transcripts for this gene may imply structural remodeling to facilitate changes in gene expression that are linked with induction of the diapausing trajectory.

Newly fertilized embryos exhibit differences in transcript levels that are consistent with the later morphological and physiological differences observed in the diapausing and escape embryo trajectories. The sequence for 6-phosphofructo-2kinase/fructose-2,6-biphosphatase-2 (PFKFB-2) is upregulated in newly fertilized embryos on an escape trajectory, and downregulated in escape embryos at the 14-16 somite stage. This enzyme has both phosphorylation and kinase activities, and directly regulates levels of fructose-2,6 bisphosphate, a potent activator of phosphofructokinase (PFK-1) and inhibitor of fructose-1,2 biphosphatase (Minchenko et al., 2003). PFK-1 is the key enzyme regulating glucose flux through glycolytic pathways, therefore higher levels of PFKB-2 would likely result in a high glycolytic/gluconeogenic ratio (Kurland and Pilkis, 1995). Expression of this protein has also been shown to be highly responsive to hypoxia and hypoxia-mimics, where hypoxia increases expression in liver and testis (Minchenko et al., 2003). At the 14-16 somite stage, this sequence is 8-fold downregulated in embryos on escape versus diapause trajectories which is consistent with a high glycolytic capacity in embryos that will enter embryonic diapause. This fits well with the observed morphology and physiology of these developmental trajectories (Chennault and Podrabsky, 2010). 
Conversely, the PFKB-2 transcript is almost 3-fold more abundant in newly fertilized embryos on the escape trajectory than embryos on the diapause trajectory. The basis of this transcriptional difference is unclear, however it is tempting to venture that this could be a form of metabolic bet-hedging in escape embryos, where rapid metabolism and development are pre-determined. The sequence for hemoglobin is also upregulated in embryos that will follow the escape trajectory. Development of circulating and respiratory pigment islands are some of the most readily apparent morphological differences between escape and diapausing embryos (Podrabsky et al., 2010). Heat shock cognate 71 is upregulated in newly fertilized embryos that will enter embryonic diapause; expression of an inducible $70 \mathrm{kDa}$ heat shock protein was found to be elevated in embryos of A. limnaeus during diapause II in an earlier study (Podrabsky and Somero, 2007). Two sequences for genes involved in cell cycle were also downregulated in newly fertilized embryos that will enter embryonic diapause; Cyclin-dependent kinase 2-interacting protein and cyclin G1- like isoform 2. Downregulation of these proteins would be consistent with the slow progression though the cell cycle that is observed in embryos developing along the diapausing trajectory.

Hormone levels of $17 \beta$-estradiol decline as female A, limnaeus age, following the same pattern as escape embryo production. There is a significant correlation between estrogen and testosterone ratios and the production of escape embryos. This relationship suggests differences in aromatase activity, as well as possible receptor dynamics. There are concurrent transcriptional differences in tissues of female fish 
producing embryos on altered developmental trajectories, in many processes that are hormonally responsive. Genes involved in epigenetics have been identified in the production of embryos on altered developmental pathways, fitting the often rapid temporal change seen in the type of embryos produced. These epigenetic changes are likely responsive to the hormonal environment, further being affected by the nutritional and environmental status of the fish. This is also seen in qualitative and quantitative differences in maternally packaged transcripts of embryos on different developmental pathways. While there is maternal control over embryonic developmental pathway, the immediate environment of the embryo during development also determines ultimate trajectory. Incubation at $30^{\circ} \mathrm{C}$, that initiates the escape phenotype, also results in a more rapid metabolism of hormones in escape embryos, and the initiation of embryonic hormone synthesis at an earlier developmental stage (5-10 somites vs 14-16 somites). This initiation of hormone synthesis coincides temporally with the critical period that temperature treatment induces the majority of embryos to escape. At this same point we also see transcriptional differences in genes involved in metabolism, epigenetics, and processes associated with the morphological and physiological development of escape embryos. Further, hormone treatment induces the majority of embryos to escape embryonic diapause. Taken together, these experimental results strongly suggest that hormonal dynamics regulate developmental pathways, both during oocyte development and through embryonic development in A. limnaeus. Future studies in receptor dynamics and aromatase activity will likely further clarify the 
components of this remarkable developmental program, and may elucidate basic mechanisms of embryonic development not apparent in a system of continuous embryonic development. 


\section{References}

Alekseev, V. and Lampert, W. (2001). Maternal control of resting-egg production in Daphnia. Nature, 414, 899-901.

Alekseev, V. and Lampert, W. (2004). Maternal Effects of Photoperiod and Food Level on Life History Characteristics of the Cladoceran Daphnia Pulicaria Forbes. Hydrobiologia, 526, 225-230.

Balthazart, J., Baillien, M., and Ball, G. F. (2001). Phosphorylation processes mediate rapid changes of brain aromatase activity. The Journal of Steroid Biochemistry and Molecular Biology, 79, 261-277.

Balthazart, J., Baillien, M., Charlier, T. D., and Ball, G. F. (2003). Calciumdependent phosphorylation processes control brain aromatase in quail. European Journal of Neuroscience, 17, 1591-1606.

Barannikova, I. A., Dyubin, V. P., Bayunova, L. V., and Semenkova, T. B. (2002). Steroids in the control of reproductive function in fish. Neuroscience and Behavioral Physiology, 32, 141-148.

Bardet, P. L., Horard, B., Robinson-Rechavi, M., Laudet, V., and Vanacker, J. M. (2002). Characterization of oestrogen receptors in zebrafish (Danio rerio). Journal of Molecular Endocrinology, 28, 153.

Bayunova, L. V., Barannikova, I. A., Dyubin, V. P., Gruslova, A. B., Semenkova, T. B., and Trenkler, I. V. (2003). Sex steroids concentrations in Russian sturgeon (Acipenser guel denstaedti $\mathrm{Br}$.) serum and coelomic fluid at final oocyte maturation. Fish Physiology and Biochemistry, 28, 325-326.

Brooks, S., Tyler, C. R., and Sumpter, J. P. (1997). Egg quality in fish: what makes a good egg? Reviews in Fish Biology and Fisheries, 7, 387-416.

Butcher, R. A., Fujita, M., Schroeder, F. C., and Clardy, J. (2007). Smallmolecule pheromones that control dauer development in Caenorhabditis elegans. Nature Chemical Biology, 3, 420-422.

Cáceres, C. E. (1998). Interspecific variation in the abundance, production, and emergence of Daphnia diapausing eggs. Ecology, 79, 1699-1710.

Callard, G. V., Tchoudakova, A. V., Kishida, M., and Wood, E. (2001). Differential tissue distribution, developmental programming, estrogen regulation and promoter characteristics of cyp19 genes in teleost fish. Journal of Steroid Biochemistry and Molecular Biology, 79, 305-314. 
Chennault, T. and Podrabsky, J. (2010). Aerobic and anaerobic capacities differ in embryos of the annual killifish Austrofundulus limnaeus that develop on alternate developmental trajectories. Journal of Experimental Zoology, 9, 587-596.

Cochran, R. C., Zabludoff, S. D., Paynter, K. T., DiMichele, L., and Palmer, R. E. (1988). Serum hormone levels associated with spawning activity in the mummichog, Fundulus heteroclitus. General and Comparative Endocrinology, 70, 345-354.

Dausmann, K. H., Glos, J., Ganzhorn, J. U., and Heldmaier, G. (2004). Physiology: hibernation in a tropical primate. Nature, 429, 825-826.

Denlinger, D. L. (1979). Pupal diapause in tropical flesh flies: environmental and endocrine regulation, metabolic rate and genetic selection. Biological Bulletin, 156, $31-46$.

Denlinger, D. L. (1976). Preventing insect diapause with hormones and cholera toxin. Life Sciences, 19, 1485-1489.

Dhillon, N. and Kamakaka, R. T. (2000). A Histone Variant, Htz1p, and a Sir1plike Protein, Esc2p, Mediate Silencing at HMR. Molecular Cell, 6, 769-780.

Drinkwater, L. E. and Crowe, J. H. (1987). Regulation of embryonic diapause in Artemia: Environmental and physiological signals. Journal of Experimental Zoology, 241, 297-307.

Dubal, D. B., Kashon, M. L., Pettigrew, L. C., Ren, J. M., Finklestein, S. P., Rau, S. W., and Wise, P. M. (1998). Estradiol Protects Against Ischemic Injury. Journal of Cerebral Blood Flow and Metabolism, 18, 1253-1258.

Duerr, J. M. and Podrabsky, J. E. (2008). Bioenergetic profiles of isolated mitochondria from diapausing embryos of the annual killifish Austrofundulus limnaeus. FASEB Journal., 22, 757.29.

Elf, P. K., Lang, J. W., and Fivizzani, A. J. (2002). Dynamics of yolk steroid hormones during development in a reptile with temperature-dependent sex determination. General and Comparative Endocrinology, 127, 34-39.

Falkenstein, E., Tillmann, H. C., Christ, M., Feuring, M., and Wehling, M. (2000). Multiple actions of steroid hormones - a focus on rapid, nongenomic effects. Pharmacological Reviews, 52, 513.

Feist, G., Schreck, C. B., Fitzpatrick, M. S., and Redding, J. M. (1990). Sex steroid profiles of coho salmon (Oncorhynchus kisutch) during early development and sexual differentiation. General and Comparative Endocrinology, 80, 299-313. 
Fergusson-Kolmes, L. and Podrabsky, J. E. (2007). Differential effects of anoxia on heart rate in developmental stages of the annual killifish Austrofundulus limnaeus that differ in their tolerance of anoxia. Journal of Experimental Zoology, 307A, 419423.

Fujiwara, Y. and Denlinger, D. L. (2007a). High temperature and hexane break pupal diapause in the flesh fly, Sarcophaga crassipalpis, by activating ERK/MAPK. Journal of Insect Physiology, 53, 1276-1282.

Fujiwara, Y. and Denlinger, D. L. (2007b). p38 MAPK is a likely component of the signal transduction pathway triggering rapid cold hardening in the flesh fly Sarcophaga crassipalpis. Science's STKE, 210, 3295.

Fujiwara, Y., Tanaka, Y., Iwata, K., Rubio, R. O., Yaginuma, T., Yamashita, O., and Shiomi, K. (2006). ERK/MAPK regulates ecdysteroid and sorbitol metabolism for embryonic diapause termination in the silkworm, Bombyx mori. Journal of Insect Physiology, 52, 569-575.

Genade, T., Benedetti, M., Terzibasi, E., Roncaglia, P., Valenzano, D. R., Cattaneo, A., and Cellerino, A. (2005). Annual fishes of the genus Nothobranchius as a model system for aging research. Aging Cell, 4, 223-233.

Gerisch, B. and Antebi, A. (2004). Hormonal signals produced by DAF9/cytochrome $\mathrm{P} 450$ regulate $C$. elegans dauer diapause in response to environmental cues. Development.

Gkouvitsas, T., Kontogiannatos, D., and Kourti, A. (2009). Cognate Hsp70 gene is induced during deep larval diapause in the moth Sesamia nonagrioides. Insect Molecular Biology, 18, 253-264.

Greytak, S. R. and Callard, G. V. (2007). Cloning of three estrogen receptors (ER) from killifish (Fundulus heteroclitus): Differences in populations from polluted and reference environments. General and Comparative Endocrinology, 150, 174-188.

Guppy, M. (2004). The biochemistry of metabolic depression: a history of perceptions. Comparative Biochemistry and Physiology, Part B, 139, 435-442.

Hairston Jr, N. G., Kearns, C. M., and Ellner, S. P. (1996). Phenotypic variation in a zooplankton egg bank. Ecology, 77, 2382-2392.

Hand, S. C. (1991) Metabolic dormancy in aquatic invertebrates. Advances in Comparative and Environmental Physiology. R. Gilles. Springer Verlag, 1-50.

Hargrove, G. M., Junco, A., and Wong, N. C. (1999). Hormonal regulation of apolipoprotein AI. Journal of Molecular Endocrinology, 22, 103. 
Hawkins, M. B., Thornton, J. W., Crews, D., Skipper, J. K., Dotte, A., and Thomas, P. (2000). Identification of a third distinct estrogen receptor and reclassification of estrogen receptors in teleosts. Proceedings of the National Academy of Sciences, 97, 10751.

Henderson, S. T. and Johnson, T. E. (2001). daf-16 integrates developmental and environmental inputs to mediate aging in the nematode Caenorhabditis elegans. Current Biology, 11, 1975-1980.

Hobby, A. C. and Pankhurst, N. W. (1997). The relationship between plasma and ovarian levels of gonadal steroids in the repeat spawning marine fishes Pagrus auratus (Sparidae) and Chromis dispilus (Pomacentridae). Fish Physiology and Biochemistry, 16, 65-75.

Hochachka, P. W. (1997). Oxygen-A Key Regulatory Metabolite in Metabolic Defense Against Hypoxia. Integrative and Comparative Biology, 37, 595.

Hochachka, P.W. and Somero, G. N. (2002). Biochemical adaptation: mechanism and process in physiological evolution. Oxford University Press. 1-451.

Hughes, A. L., Powell, D. W., Bard, M., Eckstein, J., Barbuch, R., Link, A. J., and Espenshade, P. J. (2007). Dap1/PGRMC1 Binds and Regulates Cytochrome P450 Enzymes. Cell Metabolism, 5, 143-149.

Imai, K., Konno, T., Nakazawa, Y., Komiya, T., Isobe, M., Koga, K., Goto, T., Yaginuma, T., Sakakibara, K., Hasegawa, K., et al. (1991). Isolation and structure of diapause hormone of the silkworm, Bombyx mori. Proceedings of the Japan Academy. Ser. B: Physical and Biological Sciences, 67, 98-101.

Inglima, K., Perlmutter, A., and Markofsky, J. (1981). Reversible stage-specific embryonic inhibition mediated by the presence of adults in the annual fish Nothobranchius guentheri. Journal of Experimental Zoology, 215, 23-33.

Iwata, K., Shindome, C., Kobayashi, Y., Takeda, M., Yamashita, O., Shiomi, K., and Fujiwara, Y. (2005). Temperature-dependent activation of ERK/MAPK in yolk cells and its role in embryonic diapause termination in the silkworm Bombyx mori. Journal of Insect Physiology, 51, 1306-1312.

Janssens, P. (1965). Phosphorylase and glucose-6-phosphatase in the African lungfish. Comparative Biochemistry and Physiology, 16, 317-319.

Jesus, E. G. D., Hirano, T., and Inui, Y. (1991). Changes in cortisol and thyroid hormone concentrations during early development and metamorphosis in the Japanese flounder, Paralichthys olivaceus. General and Comparative Endocrinology, 82, 369-376. 
Jung, D. J., Sung, H. S., Goo, Y. W., Lee, H. M., Park, O. K., Jung, S. Y., Lim, J., Kim, H. J., Lee, S. K., Kim, T. S., et al. (2002). Novel transcription coactivator complex containing activating signal cointegrator 1. Molecular and Cellular Biology, 22, 5203.

Kim, H. J., Yi, J. Y., Sung, H. S., Moore, D. D., Jhun, B. H., Lee, Y. C., and Lee, J. W. (1999). Activating signal cointegrator 1, a novel transcription coactivator of nuclear receptors, and its cytosolic localization under conditions of serum deprivation. Molecular and Cellular Biology, 19, 6323.

Kimura, K. D., Tissenbaum, H. A., Liu, Y., and Ruvkun, G. (1997). daf-2, an Insulin Receptor-Like Gene That Regulates Longevity and Diapause in Caenorhabditis elegans. Science, 277, 942-946.

Kobor, M. S., Venkatasubrahmanyam, S., Meneghini, M. D., Gin, J. W., Jennings, J. L., Link, A. J., Madhani, H. D., and Rine, J. (2004). A protein complex containing the conserved Swi2/Snf2-related ATPase Swr1p deposits histone variant H2A. Z into euchromatin. PLoS Biology, 2, 587-599.

Kurland, I. J. and Pilkis, S. J. (1995). Covalent control of 6-phosphofructo-2kinase/fructose-2, 6-bisphosphatase: Insights into autoregulation of a bifunctional enzyme. Protein Science, 4, 1023-1037.

LaMontagne, J. and McCauley, E. (2001). Maternal effects in Daphnia: what mothers are telling their offspring and do they listen? Ecology Letters, 4, 64-71.

Lassiter, C. S., Kelley, B., and Linney, E. (2002). Genomic structure and embryonic expression of estrogen receptor beta a (ER [beta] a) in zebrafish (Danio rerio). Gene, 299, 141-151.

Lee, J. W., Lee, Y. C., Na, S. Y., Jung, D. J., and Lee, S. K. (2001). Transcriptional coregulators of the nuclear receptor superfamily: coactivators and corepressors. Cellular and Molecular Life Sciences, 58, 289-297.

Levels, P.J. (1988). An experimental study of diapause in annual fishes. Katholieke Universiteit te Nijmegen, 1-179.

Levels, P. J., Van Tits, L. J. H., and Denucé, J. M. (1986). The effect of the presence of adult fishes, gonad homogenates, and embryo homogenates on the dispersion-reaggregation phase during early embryonic development of the annual fish Nothobranchius korthausae. Journal of Experimental Zoology, 240, 259-264.

Licht, P., Hayes, T., Tsai, P., Cunha, G., Kim, H., Golbus, M., Hayward, S., Martin, M. C., Jaffe, R. B., and Glickman, S. E. (1998). Androgens and masculinization of genitalia in the spotted hyaena (Crocuta crocuta). 1. Urogenital 
morphology and placental androgen production during fetal life. Journal of Reproductive Fertility, 113, 105-116.

Lomberk, G., Wallrath, L., and Urrutia, R. (2006). The heterochromatin protein 1 family. Genome Biology, 7, 228.

Machado, B. E. and Podrabsky, J. E. (2007). Salinity tolerance in diapausing embryos of the annual killifish Austrofundulus limnaeus is supported by exceptionally low water and ion permeability. Journal of Comparative Physiology B, 177, 809-820.

Marcus, N. H. (1979). On the population biology and nature of diapause of Labidocera aestiva (Copepoda: Calanoida). The Biological Bulletin, 157, 297.

Martin, R., Entchev, E. V., Kurzchalia, T. V., and Knölker, H. (2010). Steroid hormones controlling the life cycle of the nematode Caenorhabditis elegans:

stereoselective synthesis and biology. Organic Biomolecular Chemistry, 8, 739.

McCormick, M. I. (1998). Behaviorally Induced Maternal Stress in a Fish Influences Progeny Quality by a Hormonal Mechanism. Ecology, 79, 1873-1883.

Meier, M., Tokarz, J., Haller, F., Mindnich, R., and Adamski, J. (2009). Human and zebrafish hydroxysteroid dehydrogenase like 1 (HSDL1) proteins are inactive enzymes but conserved among species. Chemico-Biological Interactions, 178, 197205.

Menuet, A., Anglade, I., Flouriot, G., Pakdel, F., and Kah, O. (2001). Tissuespecific expression of two structurally different estrogen receptor alpha isoforms along the female reproductive axis of an oviparous species, the rainbow trout. Biology of Reproduction, 65, 1548.

Min, L., Strushkevich, N. V., Harnastai, I. N., Iwamoto, H., Gilep, A. A., Takemori, H., Usanov, S. A., Nonaka, Y., Hori, H., Vinson, G. P., et al. (2005). Molecular identification of adrenal inner zone antigen as a heme-binding protein. FEBS Journal, 272, 5832-5843.

Minamimoto, M., Sakakura, Y., Soyano, K., Akaba, Y., and Hagiwara, A. (2006). Plasma Sex Steroid Levels and Steroidogenesis in the Gonad of the Selffertilizing Fish Rivulus marmoratus. Environmental Biology of Fish, 75, 159-166.

Minchenko, O., Opentanova, I., and Caro, J. (2003). Hypoxic regulation of the 6phosphofructo-2-kinase/fructose-2, 6-bisphosphatase gene family (PFKFB-1-4) expression in vivo. FEBS letters, 554, 264-270. 
Moeller, G. and Adamski, J. (2006). Multifunctionality of human 17[beta]hydroxysteroid dehydrogenases. Molecular and Cellular Endocrinology, 248, 47-55.

Moore, M. C. and Johnston, G. I. (2008). Toward a dynamic model of deposition and utilization of yolk steroids. Integrative and Comparative Biology, 48, 411-418

Morley, P., Whitfield, J., Vanderhyden, B., Tsang, B., and Schwartz, J. (1992). A new, nongenomic estrogen action: the rapid release of intracellular calcium. Endocrinology, 131, 1305-1312.

Myers, G. S. (1952). Annual Fishes. Aquarium Journal, 23, 125-141.

Ogg, S., Paradis, S., Gottlieb, S., Patterson, G. I., Lee, L., Tissenbaum, H. A., and Ruvkun, G. (1997). The Fork head transcription factor DAF-16 transduces insulin-like metabolic and longevity signals in C. elegans. Nature, 389, 994-999.

Patel, D. S., Fang, L. L., Svy, D. K., Ruvkun, G., and Li, W. (2008). Genetic identification of HSD-1, a conserved steroidogenic enzyme that directs larval development in Caenorhabditis elegans. Development, 135, 2239-2249.

Peluso, J. J. (2006a). Multiplicity of Progesterone's Actions and Receptors in the Mammalian Ovary. Biology of Reproduction, 75, 2-8.

Peluso, J. J. (2006b). Progesterone Membrane Receptor Component 1 Expression in the Immature Rat Ovary and Its Role in Mediating Progesterone's Antiapoptotic Action. Endocrinology, 147, 3133-3140.

Peluso, J. J., Romak, J., and Liu, X. (2007). Progesterone Receptor Membrane Component-1 (PGRMC1) Is the Mediator of Progesterone's Antiapoptotic Action in Spontaneously Immortalized Granulosa Cells As Revealed by PGRMC1 Small Interfering Ribonucleic Acid Treatment and Functional Analysis of PGRMC1 Mutations. Endocrinology, 149, 534-543.

Persky, A. M., Green, P. S., Stubley, L., Howell, C. O., Zaulyanov, L., Brazeau, G. A., and Simpkins, J. W. (2000). Protective Effect of Estrogens Against Oxidative Damage to Heart and Skeletal Muscle In Vivo and In Vitro. Proceedings of the Society for Experimental Biology and Medicine, 223, 59-66.

Pietras, R. J. and Szego, C. M. (1975). Endometrial cell calcium and oestrogen action. Nature, 253, 357-359.

Pietras, R. J. and Szego, C. M. (1977). Specific binding sites for oestrogen at the outer surfaces of isolated endometrial cells. Nature, 265, 69-72. 
Piferrer, F. and Blázquez, M. (2005). Aromatase distribution and regulation in fish. Fish Physiological Biochemistry, 31, 215-226.

Pikulkaew, S., De Nadai, A., Belvedere, P., Colombo, L., and Dalla Valle, L. (2010). Expression analysis of steroid hormone receptor mRNAs during zebrafish embryogenesis. General and Comparative Endocrinology, 165, 215-220.

Podrabsky, J., Tingaud-Sequeira, A., and Cerdà, J. (2010). Metabolic Dormancy and Responses to Environmental Desiccation in Fish Embryos. Dormancy and Resistance in Harsh Environments, 203-226.

Podrabsky, J. E. (1999). Husbandry of the annual killifish Austrofundulus limnaeus with special emphasis on the collection and rearing of embryos. Environmental Biology of Fishes, 54, 421-431.

Podrabsky, J. E., Carpenter, J. F., and Hand, S. C. (2001). Survival of water stress in annual fish embryos: dehydration avoidance and egg envelope amyloid fibers. American Journal of Physiology-Regulatory, Integrative and Comparative Physiology, 280, 123.

Podrabsky, J. E. and Hand, S. C. (2000). Depression of protein synthesis during diapause in embryos of the annual killifish Austrofundulus limnaeus. Physiological and Biochemical Zoology, 73, 799-808.

Podrabsky, J. E., Hrbek, T., and Hand, S. C. (1997). Physical and chemical characteristics of ephemeral pond habitats in the Maracaibo basin and Llanos region of Venezuela. Hydrobiologia, 362, 67-77.

Podrabsky, J. E. and Somero, G. N. (2007). An inducible 70 kDa-class heat shock protein is constitutively expressed during early development and diapause in the annual killifish Austrofundulus limnaeus. Cell Stress \& Chaperones, 12, 199.

Podrabsky, J. E. and Somero, G. N. (2004). Changes in gene expression associated with acclimation to constant temperatures and fluctuating daily temperatures in an annual killifish Austrofundulus limnaeus. Journal of Experimental Biology, 207, 2237-2254.

Podrabsky, J. E. and Hand, S. C. (1999). The bioenergetics of embryonic diapause in an annual killifish, Austrofundulus limnaeus. Journal of Experimental Biology, 202, 2567.

Podrabsky, J. E., Clelen, D., and Crawshaw, L. I. (2008). Temperature preference and reproductive fitness of the annual killifish Austrofundulus limnaeus exposed to constant and fluctuating temperatures. Journal of Comparative Physiology A, 194, 385-393. 
Podrabsky, J. E., Garrett, I. D. F., and Kohl, Z. F. (2010). Alternative developmental pathways associated with diapause regulated by temperature and maternal influences in embryos of the annual killifish Austrofundulus limnaeus. Journal of Experimental Biology, 213, 3280-3288.

Rahman, M. and others (2000). Correlation between plasma steroid hormones and vitellogenin profiles and lunar periodicity in the female golden rabbitfish, Siganus guttatus (Bloch). Comparative Biochemistry and Physiology Part B: Biochemistry and Molecular Biology, 127, 113-122.

Revelli, A., Massobrio, M., and Tesarik, J. (1998). Nongenomic Actions of Steroid Hormones in Reproductive Tissues. Endocrinology Reviews, 19, 3-17.

Rinehart, J. P., Li, A., Yocum, G. D., Robich, R. M., Hayward, S. A., and Denlinger, D. L. (2007). Up-regulation of heat shock proteins is essential for cold survival during insect diapause. Proceedings of the National Academy of Sciences, 104, 11130.

Rockey, S. J., Miller, B. B., and Denlinger, D. L. (1989). A diapause maternal effect in the flesh fly, Sarcophaga bullata: transfer of information from mother to progeny. Journal of Insect Physiology, 35, 553-558.

Rohe, H. J., Ahmed, I. S., Twist, K. E., and Craven, R. J. (2009). PGRMC1 (progesterone receptor membrane component 1): A targetable protein with multiple functions in steroid signaling, P450 activation and drug binding. Pharmacology \& Therapeutics, 121, 14-19.

Rottiers, V., Motola, D. L., Gerisch, B., Cummins, C. L., Nishiwaki, K., Mangelsdorf, D. J., and Antebi, A. (2006). Hormonal control of C. elegans dauer formation and life span by a Rieske-like oxygenase. Developmental Cell, 10, 473482.

Samuel Bradford, C. and Taylor, M. H. (1987). Semilunar changes in estradiol and cortisol coincident with gonadal maturation and spawning in the killifish Fundulus heteroclitus. General and Comparative Endocrinology, 66, 71-78.

Sato, Y., Oguchi, M., Menjo, N., Imai, K., Saito, H., Ikeda, M., Isobe, M., and Yamashita, O. (1993). Precursor polyprotein for multiple neuropeptides secreted from the suboesophageal ganglion of the silkworm Bombyx mori: characterization of the cDNA encoding the diapause hormone precursor and identification of additional peptides. Proceedings of the National Academy of Sciences of the United States of America, 90, 3251. 
Schwabl, H. (1993). Yolk is a source of maternal testosterone for developing birds. Proceedings of the National Academy of Sciences of the United States of America, 90, $11446-11450$.

Shimizu, Y., Yarborough, C., and Osawa, Y. (1993). Competitive product inhibition of aromatase by natural estrogens. The Journal of Steroid Biochemistry and Molecular Biology, 44, 651-656.

Sisneros, J. A., Forlano, P. M., Knapp, R., and Bass, A. H. (2004). Seasonal variation of steroid hormone levels in an intertidal-nesting fish, the vocal plainfin midshipman. General and Comparative Endocrinology, 136, 101-116.

Suchanek, M., Radzikowska, A., and Thiele, C. (2005). Photo-leucine and photomethionine allow identification of protein-protein interactions in living cells. Nature Methods, 2, 261-268.

Swaminathan, J. (2005). The role of histone H2Av variant replacement and histone $\mathrm{H} 4$ acetylation in the establishment of Drosophila heterochromatin. Genes \& Development, 19, 65-76.

Tatar, M., Bartke, A., and Antebi, A. (2003). The endocrine regulation of aging by insulin-like signals. Science, 299, 1346.

Terzibasi, E., Valenzano, D. R., Benedetti, M., Roncaglia, P., Cattaneo, A., Domenici, L., and Cellerino, A. (2008). Large Differences in Aging Phenotype between Strains of the Short-Lived Annual Fish Nothobranchius furzeri. PLoS ONE, 3, e3866.

Tesarik, J. and Mendoza, C. (1995). Nongenomic effects of 17 beta-estradiol on maturing human oocytes: relationship to oocyte developmental potential. Journal of Clinical Endocrinology \& Metabolism, 80, 1438.

Tomlinson, J. W., Walker, E. A., Bujalska, I. J., Draper, N., Lavery, G. G., Cooper, M. S., Hewison, M., and Stewart, P. M. (2004). 11 \{beta\}-Hydroxysteroid Dehydrogenase Type 1: A Tissue-Specific Regulator of Glucocorticoid Response. Endocrinology Reviews, 25, 831-866.

Valdesalici, S. and Cellerino, A. (2003). Extremely short lifespan in the annual fish Nothobranchius furzeri. Proceedings of the Royal Society B: Biological Sciences, 270, S189-S191.

Walker, G. P. and Denlinger, D. L. (1980). Juvenile hormone and moulting hormone titres in diapause-and non-diapause destined flesh flies. Journal of Insect Physiology, 26, 661-664. 
Wang, Q., Hong, W., Chen, S., and Zhang, Q. (2008). Variation with semilunar periodicity of plasma steroid hormone production in the mudskipper Boleophthalmus pectinirostris. General and Comparative Endocrinology, 155, 821-826.

Watters, J. J., Campbell, J. S., Cunningham, M. J., Krebs, E. G., and Dorsa, D. M. (1997). Rapid Membrane Effects of Steroids in Neuroblastoma Cells: Effects of Estrogen on Mitogen Activated Protein Kinase Signalling Cascade and c-fos Immediate Early Gene Transcription. Endocrinology, 138, 4030-4033.

Webb, M. A., Feist, G. W., Foster, E. P., Schreck, C. B., and Fitzpatrick, M. S. (2002). Potential classification of sex and stage of gonadal maturity of wild white sturgeon using blood plasma indicators. Transactions of the American Fisheries Society, 131, 132-142.

Willsie, J. K. and Clegg, J. S. (2001). Nuclear p26, a small heat shock/ $\{$ alpha $\}$ crystallin protein, and its relationship to stress resistance in Artemia franciscana embryos. Journal of Experimental Biology, 204, 2339-2350.

Wourms, J. P. (1972a). Developmental biology of annual fishes. I. Stages in the normal development of Austrofundulus myersi Dahl. Journal of Experimental Zoology, 182, 143-168.

Wourms, J. P. (1972b). The developmental biology of annual fishes. II. Naturally occurring dispersion and reaggregation of blastomeres during the development of annual fish eggs. Journal of Experimental Zoology, 182, 169-200.

Wourms, J. P. (1972c). The developmental biology of annual fishes. III. Preembryonic and embryonic diapause of variable duration in the eggs of annual fishes. Journal of Experimental Zoology, 182, 389-414.

Xu, J. and O'Malley, B. W. (2002). Molecular mechanisms and cellular biology of the steroid receptor coactivator (SRC) family in steroid receptor function. Reviews in Endocrine \& Metabolic Disorders, 3, 185-192.

Yamashita, O. (1996). Diapause hormone of the silkworm, Bombyx mori: structure, gene expression and function. Journal of Insect Physiology, 42, 669-679.

Yuan, X., Yang, H., Wang, L., Zhou, Y., Zhang, T., and Liu, Y. (2007). Effects of aestivation on the energy budget of sea cucumber Apostichopus japonicus (Selenka) (Echinodermata: Holothuroidea). Acta Ecologica Sinica, 27, 3155-3161.

Zdarek, J. and Denlinger, D. L. (1975). Action of ecdysoids, juvenoids, and nonhormonal agents on termination of pupal diapause in the flesh fly. Journal of Insect Physiology, 21, 1193-1197. 
Zhang, L., Kanda, Y., Roberts, D. J., Ecker, J. L., Losel, R., Wehling, M., Peluso, J. J., and Pru, J. K. (2008). Expression of progesterone receptor membrane component 1 and its partner serpine 1 mRNA binding protein in uterine and placental tissues of the mouse and human. Molecular and Cellular Endocrinology, 287, 81-89. 


\title{
Appendix A
}

\author{
Table 1 sequences
}

\begin{abstract}
ALD23E05
TTGTGTTGGTACCCGGGAATTCGGCCATTACGGCCGGGGAGTACCAGCTCAACGACTCTG CATCCTACTACCTGAATGACCTGGAGAGGATAGCCAAATCCGACTACATCCCCACACAGC AAGACGTGCTGCGAACTCGCGTCAAGACCACGGGCATCGTGGAAACGCACTTCACCTTCA AACAACTTAACTTCAAGATGTTTGATGTTGGAGGTCAGCGGTCAGAAAGGAAGAAGTGGA TTCACTGCTTCGAGACGGTCACGGCCATCATCTTCTGTGTAGCCATGAGCGCTTATGATC TGGTTCTGGCTGAGGATGAGGAGATGAACCGGATGCACGAGAGCATGAAGCTGTTTGACT CCATCTGCAACAACAAGTGGTTCACGGAGACCTCCATCATCCTGTTCCTCAACAAGAAGG ACCTGTTTGAGCAGAAGATCATCCAGAGCCCCCTGACTATCTGCTTCCCTGAGTACACTG GTCCCAACAAGTACGACGAGGCCGCGGCTTACATTCAGACCAAATTCGAGGACCTGAACA AGAAGAAGGAGACGAAGGAAATCCATACCCACTTCACCTGCGCCACTGACACCAAGAATG TGCAGTTCGTGTTCGATGCCGTCACTGACGTCATCATTAAGAACAACCTGAAGGACTGCG GCCTGTTCTAAAAACATTTCAGAGCGTCATGATTACTTGAATAGTTCTGATGACCAGGAT GAGGTGAGGGAGAGACAACACACATCATGAGTGACTG
\end{abstract}

\begin{abstract}
ALD39F08
GTTGGTACCCGGGAATTCGGCCATTACGGCCGGGGGAGACATGAAATTGGGCAGAAGCGT CGGGGTCTTTGTTCTGATGAATGTATTCCTCTCAATGGACCGCGGCCATTTACAATCTCG TCTCAAACTCAAACTGATTCATTTTCTCCTCCGGCTACATTTGTTTGTTTTTCTGTTTCA AAACGGCAGCAAAGACTCCCAGGTTACGTTCGGAATCATGGATGGAGATCAAACAGGACA GTTTTGCCTTTTTTTAGCTGTTTGTTATTTTATTATTATTTTAGTTTTTTATTCTTGAGA AAATTCAAATGTATAGTGACGACGTGGCGGGAGGAGGAAGAAAAAAAACTGTTCGTGATA AACACTGCAAAAGAATGATTGGAGCGAAATTCTATGATTAGACTAATTTAGTCGAAAGGT TTAAATAGTGTATTAAATTAGGTTTTATTTTTCTGTATAGATTCATTTTAATTATTGTAG ATGATTCTTTATCCTTGTAGCAAGAAGACGGTTCCTAAAAGTGTAAAAGGAGCACTTTTC TATCATTTTTAACAGTAACGGTTTTATTCTTTCTGACAAAATTCTTCTATTAACCTGAAT TATGGATTATGCTTTGGTCCGGTTGTTTCAGAAAAATCCAATATGGTTTAAAATGAATTG TACATACGGATAGGTAAAATGTTTTCTTTCT
\end{abstract}

\begin{abstract}
ALD14B16
GTTGGTACCCGGGAATTCGGCCATTACGGCCGGGTTATACAAGGGCTTCAGTGGCAAAGC TGAGTTGGCTAATTCATCTTTTACTGAGTTGGACGACCATCACCATCTGGGCCACGTAGA TGCTGCCCTCCGTATGCACTACAAAGATGATCCCACCCACCATGACCACATCTTCCTCTT TTTGGACAACAAAGTATTTTCTTACTACCAACACAAGCTGGAGGTAGGGTATCCCAAGGA AATCTCCGAAGTCTTTCCTGGAATCCCAAACCACCTGGATGCTGCTGTGGAGTGTCCTAA TCCAGACTGTGATGAGGACTCTGTCATATTCTTCAAGGGAGATGACATCTACCACTTTGG TATTAAAAGCAAGGAAGTAGTGGAGAAAACATTTGAGAACATGCCCAACTGCACCGTGGC TTTCCGGTACTTGGGTCACTATTACTGCTTCCACGGCCACAAGTTCTCCAAGTTTGACCC AAAAACTGGTGAGGTCCATGGGAAATACCCCAAAGAGATTCGTGGCTACTTCGTGAGGTG CCCAAAGTTTGGTGATGACAGTAATCATCTGGACAGAGAGCGCTGCAGCCATGTTCACCT GGACGCCATCACGTCTGATGATGCTGGTAACATTTACGCCTTCAGAGGCCACCATTTTAT TCGTGAAGACGCATATAAAAACATCACTGTTGACACCATTG
\end{abstract}




\begin{abstract}
ALD41M22
GTTGGTACCCGGGAATTCGGCCATTACGGCCGGGGCTATTTCAACTACAATGGACAGATT GTGGGGTTTTTTTTGCCTAAAGAAAAGGGTTATGCTTTCAGCACGCTAACTTATTAGTTG GATCGTAATTATTTCAAGCCTCCCAAATGACTCATATAGAGATTTTAGAAAATAAGTCAG ATTTAATCACCAGTGTGTGTGTGTGGGGTGGCGGGTATGTATGTGTGCTTTCTTCATTAA AAACACAATAAATATTTATTTCACCAGAGCAGAGATTATAATTAGCCACTGGAAGCTTGT ACATGAATAACACGCCATTTAACATGACTGTGACACATATAGCTCTTTGCTATATGCTGC TTGGAGCTTGTCAGAGGAAAATTACTGTTTAATCATGTTCATTTTAAAACTTTGTTATGC AGTTTATCAATATTTTATACAAAAAAATATTAAATAAAAGAAACATTTACAGCATGGCCT TCTTCTATGGCTCACATCTCGAATGTGCCTCCTGGGCATGTGCAAAACAAACAGGTAATT AAGATATAACAGCGATCCCTTCTAGGAAAACCATGCCTCAAGAGCTCTGGCAGAAAGAAA TGCAATCAACATTTTCCATTAGATTTACAGTTTCAAATGAACCTATTTACATATATAAAT CTTATTGCTGAAA
\end{abstract}

\begin{abstract}
ALD05H19
GTTGGTACCCGGGAATTCGGCCATTACGGCCGGGGGCAGGTGGAGAAGGCAGAGCAGCAG GAGGAAAAGAGGCAAGCAAAGGGAGGCGCTTGTGCAGCGGTGAGGTGATAGAGGAGAAGG TCGGCGGGGAGTTCGACATCGTTCTTGAACTTGGGCAACCACGAGTGGGGAGAAGCAGGC CCCAGCACGACCTCGAGGCGGAGAGGAACTGCAGCGTCCGCAGACAGGCATAATGAAGGT GGGGGTCAGTAATACCTTGTGTTCTGCCAGCCAAAGTCTCATCCCAGCTTCTCAGAAGTA GCATGGTGGAGCGGCCAGGGACTCCTCTCTCCCTCTCATAGCCTCGGACTGAGGGAGGCT GAAAGCAGTCTTAGGTAAAATCCTCAAACGGTCCAGAGGACAGAGCAACGGTTTCGTGAT AATCGCACTCCACAGGAGCAGCTTCACTTGTGTTTCTTAGAAAAGGTGCTCAGGGAATCT TGTTTTTCTGATGTCCGCCACTCTGTTATTGCAGAAGGGAACAAGAAGGACAGAGGGTCA GTAGCAGGTCACCATCACTTAGTTGCTAATAAAGCCACGCACTGCTTTGGCATCTCCAAG CTGCTCGCTTTCAAAACAAATACAGCAGACCAAAGTGGAGGGGGGATTTAAATAC
\end{abstract}

\begin{abstract}
ALD40N06
GTTGGTACCCGGGAATTCGGCCATTACGGCCGGGAACTGAGCCGCGCGCAGGAGACTGAA GCACCCACCAAGATGGCCGACCGCACGGCGTCTGATCCGAACACAAACATCGTCAGAGTC AGAGACTTCCTGCTGAAACCTCTCAGAGCTCACCTGTGCCGGTTCATCTCTTCATTTCAA CTTTGACCTGTGATTTCTCGTGTTTATGACTCCTAAATGTGCCATTTGTTTGTTAGGACG TGAAAATAAACAAGTAGATTTTTATATCTTTAAATAGAAAAGGTGCCATGTTGGGTTGGG AACTCGTCCCGCCTGATGAGATTATTATTTTTGCATTAATGTAAAACTGATAAAGCTTTG GGGAAACAGGGTGAAATGTTTATTCAGCTGACACGTTTTTAAGTCACGTGACTTTTTTAT TATTTTAAATTTACCTGTAAAAAGCTGCTACTTCAACCTTTACTGCTTATATAGAAAAGA TTCTCTTCTCAGTTTAAGTTTCACCGCAGTGGAATAAAAGTCCCAAAACACTCGAGGCTT TTTGGACCCGGAGCCTAAATGTAGATATATAGATTTTATTGCATCGTGATGAAATACGAA GCTGGAACTGCAGGATTTAAGCTCGACTGCATCGTACTTTATATGATTTGTTTGCACAAC TCTCAGGTGATTGAAGCATTTTAGTCTCTGAAGAACTGGAATGTTGTGTAATGATTTTAA TTCTGCGTTTTGCTTT
\end{abstract}

\title{
ALD11N08
}

GTTGGTACCCGGGAATTCGGCCATTACGGCCGGGCAGCTTTTTGTTCTCTGCCTTTAAAG TGTCTGTGACGTCAAATTTTTGTAATACATATGAATGCGTTTTTAGAAAGAGAACACTGA GAACATATTGCAAAACAAATTTCATGTCACTGAGATGAACAGTTGTTGAGATATTTGCAT 
GTGAAGTAACAGGAAGTGATGTCAGAGGGGAATGTCTGCATGTAAACAGAGCAACAACAG CTGAAAACAGACTAGAGACAAATCTGTTTCTTTAGATTATTTTTAGACACTATCAGACAG ATGAACACTGAGTTCAAATTGTTTTAATAAAAACAAGACCAAAGAGTTTTCTTTGTTAAA GAGCTCCCCTTGGATGTCACTTCCTGTTTCTGATGTCACTTCCCGTTTCACATTAGCTGA GCCACATTCAAAAGAAAATATGCAGTTATGGGACAAAATAGGTAAAATCATGTGTGTTGT TCCAACTTTCTTAAATATAAAGATGTTTTCTTTGACTTTATGTATTTGAAGGATATGAGT CACAGGCTCTTTAAAGTTTTACAAGGAGAAAACGTTTGATTTCAAAATAAAAGTCCAGTT GGTTTTTGTTTTTTGTTTTTTTCAAACAGAAATAAAAAAAGTGCAAATATTAAAAATAAT AATAATAAAA

\title{
ALD37010
}

GTTGGTACCCGGGAATTCGGCCATTACGGCCGGGTTACGGCCGGGTATGGGCCCGACGCT CCAGCGCCATCCATTTTCAGGGCTAGCTGATTCGGCAGGTGAGTTGTTACACACTCCTTA GCGGATTCCGACTTCCATGGCCACCGTCCTGCTGTCTATATCAACCAACACCTTTTCCGG GGTCTGATGAGCGTCGGCATCGGGCGCCTTAACCCGGCGTTCGGTTCATCCCGCAGCGCC AGTTCTGCTTACCCTCCGCGGCCCACCCTTCTGATTCTCAGGCCTGACCCCGAGATACCA GGCGTGTCTGAACCCGGCATCCTGGGATTCGAAAGCTTCCCAGACACAGCCTCAGCTAAC TGTGCCCCACCAACTGCAGGGCAGAGCCTGGTGTCTAAACTCTTTGCTGAGGATGACCTG TTTAAGCCTGGCAACAAATTCTAAATCTCAAACACAGACTTTAAAAAACAATTTCAGTTT CATACATCTGCATTTACCACTCTGATTGTCAGTTTATTACAGACTAACAGTCGTTCATCA TCTGTAACTTTTTTGCCTCCTCACAAATTAATAAATATTATAAAAATCCT

\begin{abstract}
ALD16C23
TGGTACCCGGGAATTCGGCCAAAGCAGTGGTATCAACGCAGAGGACCACGCCATCGTCAC GCAAAAGAAGATGGAAATTTGTCTTTTGGGAAAATACAGACGGGTCGCACACACTGGTTT CTGTGAAAACGGCGGATCTGTTGAACCACTGTGACCTTTCTTCAAAGCTTTTCCTGTACA ACTGTGTTCCCTTTTGCTGGCGTCAGACTGATCACCCCTGTGATCTTTGTTCATCTTAAA GAGGACCATTCCTATTTTAAAATAAACTTAAAATGACAAAACGAGACAGGTCACATCTAC AGAACTGTCACCTCGAACGTCTGTCTTAAACCCAGCATTTATTTCACATTATGTTTTTCA TTGCAATCAAACAGCTTGTTTTAAAACCAGTTTTCATTTTTTTGCAGCACATTTTCTTGT AGCACATAAACCCATTTTGTTCATAAAGTTTAAAATGTACAAACAGTAGTTCTGTTGTTT GATTGTCTTAACTGTTGTGTTCTTCATCCAGTTTTTGAAATAAAACAGTCAAACTTTAAA AAAAAAAAAAAAAAAAAAAAAAAAAAAAAAAAAAAAAAAAAAAAAAAAAAAAAAAAGCCG C
\end{abstract}

\begin{abstract}
ALD18B05
TGTGTTGGTACCCGGGAATTCGGCCAAAGCAGTGGTATCAACGCAGAGTGGACAGCAGGC CGGGGTGAAACAGTTCGGTGGATTTGGAGACATGTAGAGAGGGGAAATCTTTGCGGTCTG CTTTTGTTAATAATCCTGGAATCAAGTCAGAATAAACCGTTTCCATTCATCCTGGGATGT TTGCATGTTTTTGGTGATCAGAGCCATCAGACAAAACAATTATATTTAATTTTGCCGCCT CTTTATTGTTCTTTTAAGCACTCAATAAATGTATTTTACTTATGGAAGTCTTGTTTTATG GGGATTGCTAATCTGGATACTTTCAACCCTGTGTGGTCGAAAATGTGCACCACTGAGTAA ACACATCAAATAAATGTACACTTTATTAGTTTCTGCATTTCATCAGCTCATGTCTCCAGG TTTAAAGGACGCAAAGCTTATCATTTAGGTATCAGTTCTCAGATAAGCACATGCTCAGAA AATGTAACCGCGCTTAAATTTGAAATAAACTCCATTTTAAAAGTTAAAAAAAAAAAAAAA AAAAAAAAAAAAAAAAAAAAAAAAAAAAAAAAAAAAAAAAAAAAAAAAAAAAGAAAAAAA AAAAAAAA
\end{abstract}




\begin{abstract}
ALD01F14
GTTGGTACCCGGGAATTCGGCCATTACGGCCGGGGGAATCAAGCTGAAAATATTTTGGGA CAACCACGCAGGATACTTTTCCAAGCTTTGCAGATCATGGCAGACTACGATATGATCCTG AAACACTGGGGTCCAGTGGAAGCGGATTACTCCACTTATGGAAACTTGGTTCTGACCCGC CTGTTTACCGAGCACCCAGAAACCCAGAAACTGTTCCCCAAGTTTGTCAGCATCCCTCAC GGCGACCTGGCGGGCAACGCAGCCGTCTCTGCCCATGGAGCCACCGTGCTGAAGAAGCTG GGCGAGCTGCTGAAGGCCAAGGGCAACCATGCTGCCATCCTCAAACCTCTGTCCAACAGC CACGCCACCAAGCACAAGATCCCGATCAACAACTTCAGGCTGATTTCCGAGGTGATCGTT AAAGTGATGGCAGAGAAGGCAGGGCTGGATGGCGCCGGCCAGCAGGCCCTGAGGAGCGTC ATGGCTGTGGTTATTGCTGACATGGACGCCAATTACAAAGAGTTGGGCTATGCCGGATAA ACACCGAGGTCAAGAGTCCTGCTTGGATTTTTCCAGAACCAGATAATCTTTGATTTGCAA GGGCTTTTTTTCCCCTCAAAAACACCTCTCTTTATAAGTAATCAAACTGCTGATGATAGT TATCTTATACTAA
\end{abstract}

\begin{abstract}
ALD12F21
GTTGGTACCCGGGAATTCGGCCATTACGGCCGGGGTCATCATCCACGATCAATATTTTGA TTTTTTTAAGTTCATATTTTCCTTTCTATAAAATCCTGTGATATGCTTTGCATATTGAAA CACTTCATTGGTGTTGGCTTGTATTTTTACTCTCCCAGTTTATGAGTATTGTTGTTGTCG ATGTTGCTGTTGTTCTCGCCGTTGCTGTTCGAGAAAGAGCTTCTACCAAAAGAGACATTT TTGTAACAAAAGACAAGAAAATCAACACAGTGTGTGACACACAATGCACCCTTTTATCAT GTTTAATGCAAAAAAATACTGCAAACAAAGAAGTTTAGTTAACGTCTGACATACAAACGT ACAACTTTTACTTGCTTATTTTAAAGATTTGGGGCTAATATGCATATAAAAATCAGTCAT TAGTCATTAAATGCTTCTATAAGCTGATTTTAGCTCCAATAAAAATAGTATTATGTGAAA AATCAAAGATGGAACTTGCATACATTTCATTGTTTGAAAGCGCCTTAATAATCATATTTG AACAAATGTAAAGTCATTAAAATGAGTTAAGAAAATGATGTCAACCTGTAAATTCTAGTT ATTATTGGCTTTTTTAGGTTAAAAAAGAAAATATGTAGGTGTTAATAAAACCACATTCAC TCTGACGTTCCT
\end{abstract}

\begin{abstract}
ALD06P19
GTTGGTACCCGGGAATTCGGCCATTACGGCCGGGGTGGGCATGCTGCTGGCGGGGCTCCT GGTCATCATCCCCGTCAGCTGGTCGGCCAACTCCACGGTGAGAGACTTCAACAACCCGCT GGTGGCCGCGTCCATGAAGAGAGAGATGGGAGCGTGCATCTACATCGGGCTGGGCCATCC GGCGTGCTGCTGCTCCTGGCCGGGAGGTCTGCTCTGCTG
\end{abstract}

\begin{abstract}
ALD03A14
TTGTGTTGGTACCCGGGAATTCGGCCATTACGGCCGGGCTCCTAAAATTAATTGTGGCCC CAACCATTCTTGTTCTCCATTACTTTGGCACTAGCCCTCTTAACCCTCGTCGAACGAAAA ATTATTGGCAGCATTCAATTACGTAAAGGCCCAAATATTGCTCACCCCTACGGACTATTC CAACCTTTTGCTGACGGGTTAAAACTATTTACTAAAGAACCACTTCGACCTTTAACAACC TCCCCTATTTTATTTGTCTTCATGCCTTTTTTAGCTTTTTCCTTAGCATTACTATTATGG GCCCCTTTACCTATTCCCTTCCCCTTAGCAGATTTAGACCTAGGACTTTTATTTTTACTA GCGGTCTCTAGCTTAATAGTGTATTCAATTTTAGGGGCCGGCTGAGCTTCAAACTCAATA TACGCTTTAACTGGAGCTATACGAGCAGTAGCTCAAACCATCTCATATGAAGTAAGTCTA GCCCTAACCCTCCTTAGCATTATTATCTTCTCCGGAACATTTACTATCCAAGCCCTTAAT ATTACCCAAGAGAACACTTGACTAATTATACCCATACTACCATTAATCTTCTTATGATAT ATTTCCACTTTAGCTGAGACAAACCGAGCCCCCTTTGACCTTACAGAAGGCGAATCAGAG
\end{abstract}


TTAGTTTCAGGCTTTAATATCGAATATGCAGCGGGACACTTCGCGTTTTTCTTTCTAGCC

\begin{abstract}
ALD06P03
GTTGGTACCCGGGAATTCGGCCATTACGGCCGGGG

ALD05N23

GTTGGTACCCGGGAATTCGGCCATTACGGCCGGGCAAGAAATGCTTTTCTGTGCAAACAT TTCACTTTGGAAACTTGGGCTTATCTAAAGGAATGTGTTTTATTTCCCTTCTTTTCTTAC TCTGCAGTCTAATATAAATGCTCTCCAGGACTCGAATGTAAAGAGTTTTGGTTTTCACCG TGAGTTACAACACTGCTCTGGTCTGATTACAAACAAAAACAAAACAAAATAAAAAAATTA AАAАAАAАСАTAАAАA TCCATAAAACAAAAGCCTCCAGTCATGGCGGTGTAGTGATGAAA GATTGTGTAGGAGTTTTGAGTATGTGAATCAGGATACATAGTAGATTTTGATGCATTTGT CTGCTGTATTTTTGTTTTATACACATACAAAGATAATCTTTTACTGTAAATGTACAGT

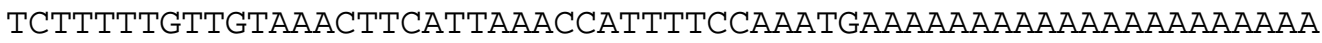
A
\end{abstract}

\begin{abstract}
ALD04P15
GTTGGTACCCGGGAATTCGGCCATTACGGCCGGGGGGAAGAGGAGATGTCACCGTCGGCG GTACAGATAGATCAGTTCATAGCATCACTCAGTTCTGTTCAAAGAAAACTCGGATATATT TGTTAGCCACACTGTGCTTGTGTTTAACATGTTGTAGCAGCGCTCCCAGTGACCAGAGCT CTCCGTCTCCAGACCGGCCGTGACAGGACAGCAGCACACAGATCAGGCCTGTTATGGCTG CCCGGCATCAGGGACCTGCAGCTGCTTCACACGGCTCAGCCGAGGCCAAAAAGACAGACC TGAGGACCAAAGAGAAGAAGTGCTCTTGGGCCTCGTACATGACCAATTCTCCCACCATGA TAGTGATGATTGGGTTGCCCGCTCGAGGGAAAACCTACATGTCCAAGAAGCTGACACGTT ACCTTAACTGGATCGGAGTGCCGACAAAGGTCTTTAATCTTGGCGAGTACCGGAGGGAAG CGGTGAAGTCGTACGAGTCCTACGATTTCTTCAGACACGACAACAAAGAAGCAATGAAAA TTAGAAAAGAGTGTGCTCTAGTAGCACTGAAGGATGTAAAGGTTTACCTGAGTGAAGAGG GAGGACAAATTGCTGTTTTTGATGCCACAAACACCACGCGAGAGAGGCGAAACCTCATCC TTAACTTTGCACAGGAAAACGCCTACAAGGTGTTTTTTGTTGAATCAGTATGTGATGATC
\end{abstract}

\begin{abstract}
ALD04H19
GTTGGTACCCGGGAATTCGGCCATTACGGCCGGGGCTTGTTGCTAACGCTACGCTTTCCG CGTCTACTGAGTAGCACTTGTCACCACTTTTTATTTATACATTTTTAGCAAGTTTGTGAG GCATGTATTTTATTTAGCACGATAAGAACAACCTAATAAGGTTTTATTTATTTTTAAAAC ATAAATTAATCGTCACTTTTTTCCCAAAACTGTTGGTTCGAAACGAAAGTTGATGTTACA TATTCGTCTGCTAGCTTACGTGACCCCTCGTGGTAATATCTCAGGTTTGATAACTCACGG GTAAATGTGGTAATACTATCTGAATTACGTGATGTATGTTCTGTGCGGAGCTGCTCGCCT CTGTCTTGTGTCTGTAAGACACAAACATCCAGCTTTGACACCGCAGCTCAGGAGGTTTGG ACACACAAAGAGGATGAAATTGTACAGCTTCCTTCAAAGTGGAAAAACAGTCAACGTGCA GACAATTTTTAGCGCAGCTATGCCTGAAAACACAAGGTGTTTGTCGTGTTCAGCTGTAGA TGCAGCATCCCGAGTGAAGAGGGTTTCTATCGAGGGTAACATAGCTGTTGGAAAGTCGAC CTTTGCAAGACTCCTGCAATCTGCTTGTTCAGACTGGGAAGTTGTGGCAGAACCTGTCAG CAAGTGGCA
\end{abstract}




\begin{abstract}
ALD41H23
GTTGGTACCCGGGAATTCGGCCATTACGGCCGGGGGCCTCTCATATATATTACCGTCCAT GAAGCTGTGCTTCCAGCATGACTTTTATGACGTCTGTTATGAGTAAAGTCAAAGTTTTTG TGATTTTTTTTTCTGAAATTTGTGCAAAGCAAGCCTAGCAGTTTTCAGTAGAAGTGCGTC CTACGTTTCTGCTCTTCGAACGATCTCCATGGTGAGATGAGCAGATGGAAGAGGCAGAGG GAGTTTTCATACAACCTCCTGTATGTTAGGTGGACGATGAGCATGAACTGTCTCATTAGC TGTGAAGTTCTTCGTTACAGATGGAGCCAGCTGGTGAATAATTACTGATGTACATGCTGT GGACAGTTTGTGCAACAATACCAGCACGGGCGAGCTGTTTGTAGTGTCTGCTCAGTTCTT TGCAGCGACTACGGGTGGGTTTAAATAATCGATGATTCAAGCATGAAGGGTGAGACACAG TACTGAGACACCCTCATATTTGATAAATGACGAGTTAGAAAACATCGGTTTGTCACGTCT CACTGCAGAACGTTACCTAATAAAAGTATCAGCTCATAATACAGGAACAATTAACTTACT GCCATGTTTAAGAATGATTTTAACTTTTTAATATTTTCTATATTTCTTTCAGGAGATCCA GCCTGGTGATCACCACAAAGCTCTTCTGGGGAGGAAAGTAAGTGTGCACTTGTTTTTC
\end{abstract}

\begin{abstract}
ALD37E03
GTTGGTACCCGGGAATTCGGCCATTACGGCCGGTGAGTGTATATTTGTTGTTTGTGATTA ACACAGTCTGATGGTAAACCATTTTTCTACCACATTTTACTATTAGGGAGTGGTGGTGCA GAGGTTAGGGAAAACGAGCCTGTGATCGAAAAGTCAATGGTTCAACTCCCTGGACCAACC AGCCCCACTCTGGGCCCTTGAGCAAGACCTGTAGCCCCTCAGCTGCTCCCCAGGTGCCTT AAATTGGCAGCACACTGCTCCACGTGTGTTCACATGTGCAAAGGATAAAATGCAGAGAAT TCATTTCTCCACCAGGGGATCATTAAAATGTATAAAAAATGCTTAATAATTGCTTAAATA AATGCTTAATAATAATTATCATCAACCTTTATGCTCACCCTGACAGAGTTGTAATGCTGA ACTTCATGGCACTTCCAATTAGCCTAGTGTGCATTTAGACCAAAAATGAACACAATTTTG CATTTGGGTAGGTATGTGACAGTAACAAAGGTTCAGTATACCCTAGTGTGTGAGAAACAC TAGTTTGTGGCACAAAAATCTGGAAAAAATTGCTGTTTTTTTTCATTAGACCATTGTGTC TGAATTGATCTGACATCAAACACATTCTCTTTGATTCTGCACGAATGCAGAAGCACATTA AAAGAAAATTGCTTGTTTCCGAACAGCTTTTGATTAAACTGAGCCTTGCTTCCATACGGT TAAAGAGTTTGTGGGTT
\end{abstract}

\begin{abstract}
ALD22E16
GTTGGTACCCGGGAATTCGGCCATTACGGCCGGGGCCTTTTGCTCCGACATCTTGACAAG GCAACATGCCTCCTAAGCAGGATAAGAAGAAGGATGCTGGGAAGTCCAAAAAGGACAAGG ACCCAGTTAACAAGTCTGGAGGCAAAGCCAAGAAGAAGAAGTGGTCCAAGGGAAAAGTGA GGGACAAGCTCAACAACCTGGTCCTCTTCGACAAGGCAACCTACGACAAGTTGTACAAGG AAGTGCCCAACTACAAGCTCATTACCCCCGCTGTCGTGTCAGAAAGGCTGAAGATCCGTG GCTCTCTAGCCAGGAACGCCCTCCAAGAACTGCTTGCCAAAGGCATGATCAAACTGGTGT CCAAACACAGAGCTCAGCTGATTTACACCCGTAACACCAAGGGTGGAGATGAAGAGGCAG CAGCAGAGAAAGCATGATCAGGTCTCCTGTTTCTGTCATCTGTTTGTAACAATGATGTAA TCAAATTGTAAAGACAAAAAAAAAAAAAAAAAAAAAAAAAAAAAAAAAAAAAAAAAAAAA AAAAAAAAAAAAAAAAAAAAAAAAAAAAAAAAATTAAAAAAAAAAAAAAAAAAAGGGCCC CCCCGCCCCA
\end{abstract}




\begin{abstract}
ALD04J15
GTTGGTACCCGGGAATTCGGCCATTACGGCCGGGAAACGAGCCGTGGAGCAAACCCAGGT CAACATCCAGTGGGTCAGCGAGAACAAAGACGTGGTCCTGCAGTGGTTCCTGACAGAAAC TGCCGACATGGAGTAACAGATCTGTGCTTCTGACAATCTGGGTCATCAAATGACTGAACA GTAAACCTGATTTCACCAGAACTTTCAGGTTTCTGTGAGGTTCAGCTGGCAGCCAGCCTG AACCACCAAGTATTGACTGTTTATGTAAATACTAATGTGCATTATTTATAGACTGTAATC ACTAAAAACAAACTTCACTTTGTTTAATAATGTTAAATGTGCTGCTCATAATTGTAAGTT GTCTGTTTAAGAAAACAGTTTTATTTCTCTTGTTGTTGCTGCCTGTGCATCAAATCGTTC CACAAACTGAACGTTTTCATGGTTTAAACATCCGTTTTCTTCCCTTCTCATAAGATGAAA TCAGTTTAAAAATCAGTTTCAAATCCTGCCGCTAATCTTTAAACATCTGTAAAAAAAAAT CTCTGCATGTTTCCACAAACAACTTCATGGTAAAGTTTTGTTATTTAACATAATTTTCAT GAACTTAATGTTAAATCTAATCAAAGATCTCAATCATTGCTGCTGCTGCTGTACTGGAAA TGTTTGAAAGAAAACATTGTTAAACATCTCTTTTCTTTCTTTTCCTGAAACCTTAAAGTT AAAACGGAAATAATGTTTTCATCTGTGTGTCT
\end{abstract}

\begin{abstract}
ALD22M16
GTTGGTACCCGGGAATTCGGCCATTACGGCCGGGATAAAAACCAGGGAAACAAGCTGATC CCTGAATGTCCAGATGAGAGGGCAATGATGTACCAGCGCATGTTTGAGGGCCTCACACTC TTCCAGAAACTGGCACAATCACTCTTCTACACACGCTACGTTCCGGAGAACGAGCGACAC GACTCTGCCCTGGAGAGGAACACAAAGGAACTGAAAGATGAGATCGGGCGATGGGAGGGA TACCTGAAGGGTAATTTCCTTGCTGGAGAGCACTTTACAATGGCTGATGTGATTGTTTAC CCAGTCATCGCTTTCCTCTTCCGCTTTGGATTATGTGAGGAGAAATATCCTAAACTGGCC GCTTACAATAACACTCTGAAGGAAAGACCCAGCATCAAAGCTTCCTGGCCCCCTACCTGG AAGGAGGGACCAGGAATGGACACTCTGAAGAACCTCTGAGCGCCTTCTGTCCACCATGTC CTCATACGTAACCACTCCTGATGTAGAAGCTAAAGCATCGGCTTTTTCTGCATGAGGCCA CTTTTCTAATATTTGTTTGTAATTTACTCTACAGAAAATGTTTTGTGCTGTGAACAAAAT ATTATTAATGAAGGTTTAATGC
\end{abstract}

\begin{abstract}
ALD07O15
GGAACTATTCGGCATTACGGCCGGGAATTAACTTAATAAATTAATTGTCATTTTTCT ACCCATATTGTAACTGTTGAAATTTACAAACCTTCACACTTCAACGATTTTCTTAAG CCTCACAGAACAACTTGGGCTAGAGAAACACTTGATTATTTAAACCTTCTTAAATGT TCTAAGCTGAGCCTATAAACACAATAAAAAAGTTTAGAACCATTAAAAAGGGGGGAG CCAACAAGTAGCTTATATAAAGGTATTACCTTTACTAAATTAATAATAACACTAATA ATACTAATCCTACTCAAAATTTTCACCTTTAAATATACCAAGCTTATTGCAATCTTA CTAGTTTGCTCTCTGGTTAACCGGTTTTCTAAAAATAGTATACCACAACCCACTTTT CCCAACATCCACACAATTTAAAATCAAAACCCCTCTTAAA TTAAATAAACTAAAAAT AAGCTAGCATAAAACACTATAAATCTACTAACACCTCTTAATAATTACTGCAAAATA TTTCTTTCCTATTTAAATGTGCATATACTAATAAATTTTACTAAAAATAATAATAAC TCTTTTCTAACCCTGTGTTAACAAACACTTTAAAGCTCACTATAACGTCAGGAACCT GTGACACAGTTACATACTTTCCCGATTTCTTTATAGTATCTTATAGCTACTAAAACC CACAACAAACTTCACCAGTGTAGTGTCACAAGTTTATTAAACAAGCTACGTTGTTAG CTCTAACTCTATTAATCTTGTAATCACACTTACATTGCAATAACAAGACTGCAAACA AAGAAAACAAAAAAAATAGGCCCCCCGCGGCCCATCGACTCTAGAGATCGAAGAAGG TATATGCTGCTGTCCGCATTTCGGGCTCACTGGCCAATGCACATGGGGGGCAGACGT GATTAGTATCATGCCCCTTAGAGTTTGAACTAAATTTTCAAAAGA
\end{abstract}




\begin{abstract}
ALD35J06
GAATTCGGCCATTACGGCCGCGGGGTTTTTTCCTTTGTGCAATAGAAGCAAAGACAAATA TCTTCATCTAAAATTTACAAGTGATTGAAAATGTCCCCCCTTTTTTTGGATTACTTCTAA ATCACTGTAGACCCCTCCCCCTCAAAAAAAGAAATACAAAAAATTATAAAACAGGATGAA ACAGAATCTCAAAATGTGATGGATTTTGGAGAATTTTTTGATGAATATGATATAAGATTA TATTAATATTGTAACAAAATGGAGTTTATTTGAATGGGAGTTTTTTAGTTTCATCACAAA AATCACAAGTGCTTATTACATGCTGGGGTGATTTGTCTGTTTTTATGTTTGCATCATTTG TTCACAGGTTTGTGGCTCTTTACCACAGTCTATTATGCATTTTAAATTTGTGATAGAATA TGCCAACAATATGCTTAATGCATTTGCAACCAAGATATACCAAATCATGTGTAGTTTTGA AATGCACCTTTAAATATTAAGCAATAAATGTTTTGTCAACATCNAAAAAAAAAAAAAAAA AAAAAAAAAAAAAAAAAAAAAAAAAAAAAAAAAAAAAAAAAAAAAAAAAAAAAAAAAAAA AAAAAAAAAAAAAAAAAAAAAAAAAAAAAAAAAA
\end{abstract}

\begin{abstract}
ALD38F08
GTTGGTACCCGGGAATTCGGCCATTACGGCCGGGGGAAGAAGTGGAGGAGAGAGGAAATG CTTCACTGAAGAAGAACCCATCCAGAGGACCTTCTTATTGTTTTCATTTTGGTTTAAATT AAATTAGGTTAATTTATCAGTAGTTTCCCCCCAAAAGGAGTACAAAAAGTTAACCAAGCC AGGAGAGATGGCAGACCGAGCCTTATCTGCTGCCAAGGAGGTTGTCCTAATGCCCAGCTG TGACCTTCCTGAGGACCTGCCCAGAATCCGAGGCTACGACTTCAACCAGGGCGTCGATCT GAAGGCAGTGCTGAAGTCCTACCTCACCACGGGCTTTCAGGCCAGCAGTTTGGGTTTAGC CATCCAGGAGTTCAATCACATGATAGAGAAGCGCCTTGAGCCAGTGGAGGTCGATGAAGA AAACAAGTCTCTGGAGTCTGGTGAATCCTCCGGAAAATCAGGCTGCACCATCTTCCTGGG CTACACCTCCAACCTCATCAGCTCCGGAGTCAGAGAGAGCTTCCGCTACCTGGCAGAGCA CAAAATGGTGGATGTGATCGTGACCACGGCGGGAGGCATCGAGGAAGATCTGATCAAGTG TTTGTCTCACACCTACCTGGGAGACTTTAGTCTGCCGGGGAAGGAGCTCCGGCTGAAA
\end{abstract}

\begin{abstract}
ALD25M22
TGGTACCCGGGAATTCGGCCATTACGGCCGGGCACCTCAGTAGAAACGAAAAAAAAGGAA GCATCTTGCACACCTGAAGTGTCCTTATTATGGATTTGCGACGGCAGAAAGATTTTATCT TCCTGCACACAAAGAAGTGCTTCTCAAAGACACAAAGACAGACTTTTTGACAAACGGCTG CAAATTCTTGTTGCACTGCGGACTTTCACGTGCAATTTTGTGCAAGCAGTTACTGTTAAA TCCTTTTTTTTTTGCTCGTTTGTACTGTTGTTGAAAAATGTCAGTGTTGGCTGCTAAAA AAAGGCTGTGAGGTCAGCAGCATTTTTTTTTATTATTATTATTTTTCTGCCTGGAAGCAG TCACTTTCTAACACGCTCCATCATGTGGGAGGAAAGCAGGCAGACAGCTCCGTGGTCATA CTACACTCCTTCTCCAGCCGATCCCCCCGCCTGCGGTGCCAGTTTCATGGCAGAGCGCTG CCTGAACAAGACTTGAGGAGTTCGTTAAGCGTTTTTCAACTAAGTGCTTTTTTTTTTACC CAAGAAGAGCTTAGCGAGTTGACAGGAATGTTGTAATCTATTTTTTTCCTGCTGTGTACC GTTGAGAACACCTTTTAAAACCCACATGTCACCCTACTTATGAATACCTACGAGATAAGT GTGCCAAATCGTGTTTTGCCAGTGGGTGTCTTAATTTATTTTTTAGTGTTTTTAAATGGT AAAACTGAAACAAAGGTAAAGGAAATTTGA
\end{abstract}

\title{
ALD08I08
}

GGGAATTCGGCCATTACGGCCGGGCAGACAATGTTGTATCATGTTTCATTCCTGCATTTT 
AACTTAAATTTTGTAATTTATTGTAGCTAGAAATCATCTTCAAAAGAAAAAAAAATCTCT GACTCAACAAGCACACTGATGTGTACAAAAACACTGCTCTGTTGATGAATATGATGTACT TCCACGTCTAGAGCTTCCTTTTAAGCAAGTGTGTTTTGCCATGTTTTTTCAACTTTTTTG CTAGCACTGTAAATTAATGCATTCCTAGGCTACTGTGAAGTCTGTTTCTTGTTGATGAGG AGCAGTCTCCTTTTGGCTCCAACTCCCTTCGGTGTTTTATATATGGTTAAAAAAATGTAG ACTATTGTGATATTGCCAAACTTGTGCTAAATATTTATACATCTGTCTTTTTCATGTTCT TTTAGATCAACTAAAAAAAGGTAAAAAAAATTATAAAATCCATTCAGAATGTAGGATAGT AGTTCATTGTCACATTTCTGTTTTAATACACACTTGCGCTCACTGAAGGAAATTTTGTAA CATATCAACTATAAATAATGTATTTATCTCTGAAATTTTGTATATTGCTTTTCATTATGT ATGTATTAATCATCATGCCCTTAATATAGTGATGCATCACAGATAATTCAACCAAGAACT GTTGCCTTCATTTGTTTTTCTTTTTGTATTT

\begin{abstract}
ALD38A07
GTTGGTACCCGGGAATTCGGCCATTACGGCCGGGTGACAGAGCTGTATCCGAGCACCAAA GACATTATCCAAACTGTCGATTTGTCCGCGGAGACAGGGCTGACAACGTGTCGCTGGCCG CCGCCGCTGCTACCGCAGCGTTAGGAGGAACGTCCCAAATGTCTGCCGGGGCTCCTACCC TCACCAACGTGTCCAACCCATCCATGCAGCAGAGCGAGGAGCGGCTGCTCACCTTTGTCA ACTGGCCGTCTCGTATTCCTGTGCGGCCTGAGCAGCTGGCTAAAGCCGGCTTCTACTATG TCGGGAGAAACGATGACGTCAAGTGTTTCTGTTGTGATGGAGGCCTGCGGTGCTGGGAGT CTGGAGACGATCCCTGGGTGGAACATGCTAAATGGTTTCCTCGGTGTGAATATTTGCTTC AAGAAAAGGGACAAGAGTTTGTTCACCAGATCCAAGCTCGATTTCCACGGCTGTTTGAAC AGCTTTTAACAAATGGAGACAACTCCAGAGAATTTATGGATCCTCCTGTGGTCCACTTGG GTCCAGGGGAGGAGCGGTCTGAAGATGCTGTCATGATGAACACCCCTGTCATTAAATCTG CTTTAGAGATGGGATTCGAGCGCAGCCT
\end{abstract}

\begin{abstract}
ALD35F16
GTTGGTACCCGGGAATTCGGCCATTACGGCCGGGGATATTGACCTTGTTGATCACACAAA CTTGATTCTTTCTGAAATTAAATCCCCCACTTGTTACCTAAAACAAAAAAGTTTCAGAAG AGCAATATTTTTTGATTGGGAGCAATTTTGATTTAAAATTATAAATTGAATGAAAGTGAT TAAATACTTTATAAATGAATGTTTTTGGATTAAGCTCATACTGACTGTAGACAAACATGA TTCATAAAAAACTTTCTTAGAGGCCTGATATTCCTTTATTAGGCCTGTCCTTTCCTGAAA TTTACCCCATTCGTTATCTAAACTGTGATATAAAATTTGTATCACAATGAATACAGTTTA GTTCAGTGTGCAAGTGAAGATAAACTGTGTAGGGTAGCAAAGCATGATGCTGTCTTTCAT TTCTGAATGTTTTTTTTGCATTCATTAAAAACCCACTGACATCTGGCTCTCGTATACTGT GGAACTTCGATTAATTAGTTTGCTTTGAGTTTTTGTAAAATTTCTATCAATCAACATCAG ATGAAATCTGCATTCATGGGAACACATCAGTGATGATGACGACTCCCAGAATCAAATGTT TATGATCAGCCTGTAATTATGGCTGAGCTTTACTGTGAATGAAAGCCGATATTAGGTATC GGGACCAATATCAGATATTGATATTGGATTGGG
\end{abstract}

\title{
ALD19J15
}

GTTGGTACCCGGGAATTCGGCCATTACGGCCGGGCATGGTGGAGCGCAGCAGGGGGGCTG TGGTCAACATATCATCAGGGCCCTGCTGTAGACCCCTTCGTGGACGAGTGGCTCTCACAG CGGTCACTGGATACTTGGATCATTTCTCTAGAGCTCTTCACCTGGAGTACAGCGACAACG GCATCTTTGTCCAAAGTCTACTACCTTTCCAGATCGCTTCAAGCAGGAGTCAACCATCAT CACCATCATCATCATCGTCAAGAGAAGGCTGGTTTGTTCCCAGTCCGGAGGTTTATGCTC GACATGCTGTCTCTACGCTGGGCGTGTCTAACAGAACCACAGGCTACTGGCCTCACACTC TGCAGTACGGCCTGACCAGGTGTGTTCCTGACTGGATCTGGATTCTTGGCTCACGGATGT 
TCATCGGTTCAAGTTAGGAGCTCAAAGTGTTGAGTTTGTTACACCAGAAACAACAAACAT GAATGACTTCAGACAATAAATTAGTTACAACAGCAGGCCCTGGTAAAAGATGTCAGTTTG TCTTGCTGGTGAAGCTTTAAAGAAATGGTACATCCATTTTTTTTTTTTAGATGAACGTTC AGCTCAGATCTGAATTCCTGTTCAAACTGCAGCAGAGCTGGGTGTAAAATTATTTGTTCA ATTCAGAGAATTGGTGAAGATCTGTAAAGCTTCAGAAACCATAAACTCAGTTTATTAAAG GTAGACAATGCTGAAAAAAACTAAAAATTAAC

\begin{abstract}
ALD33B24
GTTGGTACCCGGGAATTCGGCCATTACGGCCGGGATTACAGCCTGAACACAAGCAGCATC TCCACCCAGCCGTCTGTCACACAAACCATGGTGTCGGCGGTCATCGACGAGACCGTCCCC GTCACCATCCTGGAGCCCCAGCCGATCAACGCCGAGATAAAGACAACTCATGAGAAAAAC GTTTTGCCCGATGAGGTTCTTCAAACCTTGTTGGACCACTACTCCAACAAAGGCAACGGC CAGTCAGAGATTTCCTTTAGTGTTGCCGACACAGAGGTGACGTCGAGCATATCCATTAAT TCCTCCGAGGTTTCGGACAGCAGCCCCGTGGACAACCTCGGAGCCTCGAGCGCTCCGGCT CAGCCGCCTTCTGAGAAAGTCAGTCTTTTGCAAGAATACTCCAAATTTCTCCAGCAAGCT CTGGAGAGGACCAGTCAGAATGACAGCTACCTGACAAGCCAGAGTCTCAGTTTGGTCTCA GAAAACCCCACGTTGGTCGGACAGCCTCTGTTCTCCACAGAGAAACCGTTTTCCTCCCCC AGCAGGTTCAAATCAGGGATGAGCTCTCCACTGAGATCCACTTTAGAAAAACCTCACTTT GGATTATTGGTGGGAGACTCCCAACACTCGTTTTCCTTTTCGGGCGACGAGACCACCTTG CCCTCTGCCGTGTCCCCCTCTGGGGAGGACTTTTTAGAGGTCTCGCCTCCTAAAAAAACG GACTCCTC
\end{abstract}

\title{
ALD18F02
}

TGTGTTGGTACCCGGGAATCGGCCATTACGGCCGGGGGCCGGGGATGGGAGAAGATGG 


\title{
Appendix B
}

\author{
Table 2 sequences
}

\begin{abstract}
ALD27K10
GTTGGTACCCGGGAATTCGGCCATTACGGCCGGGGCAACAACAAAAGGGTGATTATTTTA TTAGTATATAAACATACACTATGATGAAAATGTATGTAAGAAAAAGAAAAGTTGTGAACC AGGTACTTCCTTCATCCACTGTCTGAAAACTTTCACAGCTTCTTATTGAATCAGAAACTT AATGCTGTTTAAAAAGTCACGTTACTCATTTTATTTCCACTTTTGACTTGACTCAGATCT GCGTTTGGTACGAAGACTGCAAACCAATGTCACCAAAACTACTTTGATTTCAAAATTTTC ATTATTTTTTTTTCTAACAAAGATAACATTGGTTTCTGCATTTCTTCAGATATTTTTCAT GAATTCTTTTTCGTCAGGCTTGAGTCATTGTCCTTTTTGTGTTGCTTTTTGTGCAGTTTC TGTTTATTGAAGTGACACTTTGAAAACAACTCTAGATTTTTTTTTTTTTCAAATGCAAAC TACCTATTTACTGTGGCTGGAATAAACAGGAAGCTGTGGTCACCGACACATTGTGACTCG TCGTTTTACGTGATATTTTTGTGAACTCTCGGTTCTTCGGTGCCTCACAACAGTGCTGAT TCACTTTCATCTGTAGGGCTTTTATTGTTTCTGCTGGGCTTTGAACTTTGAGCTGTACAG AATCAAAAGACTAAACAAAGATAAAAACCTTTGTGTCCCTGCACCAGCGTGGTCAAGGAG G
\end{abstract}

\section{ALD38K20}

GGGGTGCTTTGCCTGTGGATACAGATGGCTCCATCCTTGT

\begin{abstract}
ALD33F13
GTTGGTACCCGGGAATTCGGCCATTACGGCCGGGGATGATCCAGTGAAACATGCAGGGGT TTACACAGCAGAGGAAGTGGCATTGATCACTCGAGAGAAACTCATTAGACTTCAGTCTCT CTACATCGACCAGTTCAAACGTTTGCAGCACCTGCTGAAGGAGAAAAAGCGCCGATACCT TCATAACCGCAAAATGGAGCATGAGGCTTTAGGAAGTGGTCTGCTGACGGGGATCGAAGG TCTTTCCATGAGGAAAGAGAAAATCTGAAGAAGCTCAAAGCTCTGCGTCGATATCGTCGG CGGTATGGTGTGGAAGCTCTCCTGCATCGACAGCTCAGGGAGAGGAGGCAGGCTGTGACT GAAGGAACCCCTCAGCTTCATTCAAGAACACTAACTGAAAAATGCATGGCGTTTGTGGAG GGAGCCAGGTGTACCAATCCCTGCCTGCCCATGGCCCGGCACTGTATCTCACACATTTAC CAGGACAACAACCAGGTGTTGTTTAAAATTTGTCCCGGGCTTAAAGATGTTCCCTGTGAT CGAATCGTCCACATGGGTCAGTCCGATGATCCTCGCTGCCCCCTCCACCTCACTCTGCCT CCGCTCATGTACCAGCCAGAGCAGGAAGCCCCCCCTCAGGACCAGTTCACACCTGCAAGC AAAGACATGTACCTGAGTGCAGCAGAGCTGCAGCCCACAGAGAGCCTCCCCCTGGAGTTC AGTGATGACCTGGATGT
\end{abstract}

\begin{abstract}
ALD19D01
TGGTACCCGGGATTCGGCCATTACGGCCGGGGGGACCGGGGGGACCGGGGGGACCGGGTC TCAGGGTCGTGGCCCGGACCTCCCTGCGGCTCTGTCGGACCTACAGCCGAGGAGAACCGT GCTCAGACTGCCAGCAGCTGCACCTCTGCAGGTTCTTCATCTACGGAACCTGCAGGTTCG GCAAGGGCAGGAAACCCTGTAAATTCTCCCACGACATCCAGTCAGATTATAACTTCAGAT TACTGAGAGAGTGCACGCTGCATGAGCTGACTGAAGAGCAGCTCTTCCTGCTTCTGCTGC AGAATGACTCGCAGCTGCTGCCCGAGGTGTGCCTGCACTACAACAAAGGTTCAGGTCCTC ACGGTGACTGCAGCTTCCAGGAGAACTGCTCCAAAGTCCACCTGTGTCAGCACTTTGTTC
\end{abstract}


AGGGAGCCTGCATGTTCGGACACACGTGTAAACGACTACACACTATCGAGCCGCACACTC ACAGGAGGCTGGAGGAGAGAGGACTGAGTGGAGACATCATCCAAGACCTGCCCTGTATCT ACAGGAACCTCCACGTCCTCACAGCTGCTGCTGCTGCAGAAACTGATCCAGATGCAATCA TTGAAGCAGATCAAAGAGACGAGCCGCAGGAAATCTGTCTGCACTTCATGAGAAACAGTT GCAAGTTCCAGCAAAAGTGTCGCCGCATTCACTTTCACCTGCCGTACA

\begin{abstract}
ALD35A12
GTTGGTACCCGGGAATTCGGCCATTACGGCCGGGGCCCGTGTGTGTTACCTTCCGTGTTC CCCCTACGGCTCGGCTAATCATAGCTCGAGTCCAAAACTGCTCAAAAATTGCGTTTAAAC ACACCCAAAGTTCTCTGAAGCGGTTCAAACGACAAATTGCAGTGTTATCGACTGCTCAAG GCTGACGAAACGGCTTTTGTCGCCGTGAATCGAGACGTACCGTTCGGTCTTTCGTCCCGG AAGTAGCTCAAAGCTAACTATTTAGCTCTGTGTTACGCTACTCTGTTACTCCTCATGTAT TCCGTTTAACAACTTACGAGTGGGTAAACTCATAACAGATGTTAGCGGAATTATGCAAAT AAAATCTCAACTGCGTTCACAGATTTGATTTTAAAATAAAATAGCCCAGCGCTATTTATT AGAGAGGAGTATATATTCCCTTTTCAGGAAGTGACGTCATCACCTTTATAAACCGTGAAA TGCTTATATTTTTAAGCACAATCAATGTTAAAGCCTTGTTTACAACCTCAGAATGACAGA ATAACAACAAAATCTGCAGCTTTCTGCTACAATCTGACTACCTTCAGTCATAACAGGGTC TTATAACATCACTCTTTTTGCCAACATGAGAAAGTAATATTTTTTTTACTTCTTTTCAAT GCACAGAAAATATTTTTTCAT
\end{abstract}

\begin{abstract}
ALD35I18
GTTGGTACCCGCGGAATTCGGCCATTACGGCCGGGGGCCAACATGGCGGACTACGACCTG ACCACAAGAATAGCTCATTTTCTGGACCGGCACCTGGTTTTTCCTCTGCTGGAGTTTCTG TCCGTTAAAGAGATCTACAATGAGAAGGAGCTCCTGCAGGGGAAGCTGGACCTCCTCAGC GACACCAACATGGTGGACTTCGCCATGGACGTCTACAAGAACCTGTACCCGGATAAAGAG ATCCCACACTCTCTGAGGGAGAAGAGGAGCACCGTGGTGGCTCAGCTCAAGCAGCTCCAG TCAGAAACTGAACCCATCGTGAAAATGTTCGAGGACCCAGAGACCACCCGGCAGATGCAG TCCACCAGAGACGGGAGGATGCTGTTTGACTACCTGTCAGATAAACACAATTTCCGTCAG GAGTACCTGGACACGCTCTACAGGTACGCTAAGTTCCAGTATGAGAGTGGGAACTACTCC GGGGCCGCAGAGTACCTCTACTTCTTCCGTGTCCTGGTTCCCTCCACGGACCGGAACGCC CTGAGCTCTCTGTGGGGGAAACTGGCCTCAGAGATCCTGATGCAGAACTGGGAGGCAGCC ATGGAGGACCTGACCCGTCTGAGGGAGACCATCGATAACAACTCGGTCAGCTCTCCCCTC CAGTCTCTCCAGCAGAGGACCTGGCTCATCCACTGGTCCCTCTTCGTGTTCTTCAACCAC CCTAAAGGCCGGGACAACATCATCGAC
\end{abstract}

\begin{abstract}
ALD14G11
GAATTCGGCCATTACGGCCGGGGGTCACCTGACACACGGTTTTATGACTGAGAAGAAGAA AATCTCAGCGACATCTATCTTCTTTGAGTCGATGCCATATAAGGTGAATCCAGAAACTGG CTACATTGATTATGACCGACTGCAAGAAAACGCTCGACTGTTCCACCCCAAACTCATCAT CGCAGGAACAAGCTGTTATTCTCGCAACCTTGACTACGCCCGTCTGAAGCAGATTGCTGC TGAGAACGGTGCTTACCTGATGGGGGACATGGCTCACATCAGTGGATTAGTGGCTGCAGG AGTGGTGCCCTCGCCCTTTGACCATTGTGACATAGTTTCCACCACAACTCATAAGACGCT GCGTGGATGCCGTGCAGGAGTTATTTTTTATAGAAAAGGTGTAAGAAGTGTTGATGCCAA AGGAAAGGAGACTCCGTACAACCTGGAGTCTTTGATCAACCAGGCTGTGTTTCCAGGGCT GCAGGGAGGACCACACAATCATGCTATTGCAGGTGTTGCTGTGGCTCTTAAACAAGCCAT GACACCTGAGTTTAAGGCCTACCAATCACAGGTTCTGGAGAACTGCAAAGCTCTGTCCAG TACTCTTATGGATCTCGGTTACAAGATCGTCACTGGCGGTTCTGACAACCATCTGATCCT
\end{abstract}


GCTGGATCTGCGTAATAAAGGAACTGACGGCGGTCGAGCTGAGAAGGTCCTGGAGGCCTG TGC

\begin{abstract}
ALD42B03
GTTGGTACCCGGGAATTCGGCCATTATGGCCGGGCTTATGGAGCAGCTGTCCAGGCTGCC ATCCTGTCAGGTGACAAGTCTGAGAACGTGCAGGACTTGTTGCTCCTGGACGTCACACCC CTGTCTCTCGGTATCGAGACTGCTGGCGGGGTCATGACGGTCCTGATCAAACGTAACACT ACCATTCCTACCAAGCAGACCCAGACCTTCACCACCTACTCTGACAACCAGCCTGGTGTG CTCATCCAGGTGTACGAGGGTGAGCGTGCCATGACCAAAGACAACAATCTGCTGGGCAAG TTTGAGCTGACAGGCATCCCTCCTGCTCCCCGTGGTGTCCCCCAGATCGAGGTGACGTTC GACATCGACGCCAACGGTATCATGAACGTGTCTGCTGTCGACAAGAGCACCGGCAAGGAG AACAAGATCACCATCACTAACGACAAGGGTCGCCTCAGCAAGGAGGACATCGAGCGCATG GTTCAGGAAGCTGAGAAGTACAAGGCAGAGGACGATGTCCAACGTGACAAGGTGTCTGCA AAGAACGGGCTGGAGTCCTACGCCTTCGACATGAAGTCCACAGTGGAGGATGAGAAGCTT GCTGGAAAGATCAGCGATGACGATAAGCAGAAAATCTTGGACAAATGTAACGAGGTCATC AGCTGGTTGGACAAAAACCAGACTGCTGAGAAGGATGAGTATGAGCAT
\end{abstract}

\begin{abstract}
ALD14K02
GGATAACAAACAGGCGAGATCTGACAAAAATCACAAGTTCAGAGTGAGAGGATGGCAAAG TAAGAGTGGAAATAAAGTGAGAGGAGCTGAAGGCTTTACTGCACACAGAACAGGCTGACG GATCGGACAGAAAACTTAGGAAGGTGTTTCCAGATATATCTATGCAGGAGGAGCTGGAGT GGGAGAGCTAACAAGTCTAAAGATAGCATGGAGAGCAGCGGAGGGACACGGAAACAAAGA GGAACAAGGTGTTTATTTTTACACACTTTAAGCGAGTATTTGAAGGGTTCAAGCCTTAGA TGAAAACAGACACTTAAAAACTGCAATTTAAAAAGAAGAATTTAGAAATAATAATAATAA TAATCCAGATTCTCCATCAATTCGGCTCGAACAGCACCTGATCGGTCCATCTTCACGGCC GTGCCGATGAAGGAGTAGTAGCATCGATTTTTCCTCCTCGTCTCGTCCCGCTGTCTTACT TTTTCCTCCATTAGCTTCCCGACCACTTCGTCCATTACTGTTATGGAGCTAAGAGAGAAA AAAAACATCATAAAAAGTGGAGCCATCGCTTCCTCCGTTAGCTCTATCTTTCCTCCAGCC GTCTTGTTCACTCCGTCTGTTTTATCACATCTCACACACCCCTCTGCTCACAGAAAACCT $\mathrm{C}$
\end{abstract}

\begin{abstract}
ALD38D03
GTTGGTACCCGGGAATTCGGCCATTACGGCCGGGGGCTTTCGCTTTCATTTATCAGGAAA CGCATCGTTCAACAGCACAAACACGGAAAGAGTTTGTCAAAATGTCAGCGAGAACCGAAG AACCAGAGGCCATGAACGTCGACGTTCTGGCTTCATCCCAAAAAGAACCTGAAACTCTCC TCACACTCTCACTAAACTGGTTACTGTCCGATCAGCAGCCAAAAATGCAGAAGATGCTGC AGAAAGCTCTGCAGACTTGGTTCAACACAAATAAACTTAAAGATGGAATTAAGGTGGACA TAGACGTCAAAGACATCCTAGGAGACGGGAGTGCTGTGATAAAGATTAAACCTTCTCCAG CCCTGACTGATCTTCAGAAACTAAATGGTGAAACTCTGACAACAAAGGATGGGATAATAG TAGCCACCATACAGTCAGTCATCCTGGGACAACCAAAGCTGAATAAACAACCACCAGATG ATGTTTCAGTGAATTCACCTCCATCGGACAAGCCAGCTGATAAAATGCAGCTTGAAGAAC CAAGTGACATCAGTTCTGCTGCAGGACAAACTGCTGTTGATGATAAAAACCCATCTGAAG AGCGTATCATCCCAGTGAGCCATTACTGGTATATGAGCCATGTCTACAAGGATGAAATCA AACGAATAGAGAAACAA
\end{abstract}

\title{
ALD33F04
}


GTTGGTACCCGGGAATTCGGCCATTACGGCCGGGGGAAAGGGTTTATTGTTTCTTTGCTT TTCGGGAGCTGAATTTGTTTGCGATCCGGTCAGTTCTACAATCAAATAACGAGACGGAAA AATGGCTGGTGGCAAGGCAGGAAAAGACAGCGGGAAAGCCAAGGCGAAGGCGGTGTCGCG CTCCCAGAGGGCCGGGCTGCAGTTCCCGGTGGGTCGTATCCACAGACACTTGAAGACTCG TACCACCAGCCACGGTCGCGTCGGAGCGACGGCAGCGGTTTACAGCGCTGCCATCCTCGA GTACCTCACCGCCGAAGTACTAGAGTTGGCAGGAAACGCCTCCAAAGACTTGAAGGTGAA GCGTATCACTCCCCGTCACTTGCAGCTGGCAATCCGTGGTGATGAGGAGTTGGATTCTCT CATTAAGGCAACGATTGCCGGCGGAGGTGTCATCCCCCACATCCACAAATCCCTCATCGG GAAGAAGGGCCAGCAGAAAACTGCATAAACGCCACGTTGACCACAGATTTTGGGGCTTGG GCTTTGTTCGGACCAGGAGTTCACATTTGTTCTTTTCTATTATTAATGTTATAGCAAACG AAGAGTGATTGTTAGCCTTTGTGTTGTTATATTTTTCCCGTAATTAGAGGAAAGTACAGC TAATCCCTTAACAGCAGAAAAACCCTTTTTTATGTCCTTGTAGAGCGTTTGACTGAGGAG CTCATTATTACTGAAATGA

\begin{abstract}
ALD24L13
GTTGGTACCCGGGAATTCGGCCATTACGGCCGGGGAGAAGGAGCTGTTCCTACCGGCCAT GCGGAAGAAGAGCAGCTCCAACACTGCTGAGGGCTCCGTCAACGGTGACACACTGACCGC CCACTGGTTAGTGGACGACATGCTCACTTTTGAGAACGTGGGTTTCACAAAGGATGTGGG GAAAATCAAGTACCTCATCTGTGCAGATTGTGAAATTGGACCAATCGGCTGGCACTGTTT GGATGACAAAAAGAAATTCTATGTGGCTTTGGATAGGGTGAACCATGCATAATGTTTTCA TGCATATGTGAGCCCACACGGACCTCATTAAATGTTTTGGCAGGCTATGCATTGTTTTTA TGGATTTATGCCCAGTTTACCCCGAATAAGTCCATCGTACACACACTCACTGATACTGTA CTGTTAAAGGGATTGTGCCATGTTCATTTCCATCGATCATAATGGCATTAGTACAACCAT TCTGTTCTAATAAAGTTATTAATGAAGTCATTATTTGAATGTCATACTTGTTATATTTGT TACCGTCACCTAGATGAAACAGTTTAGAACAATTTACAATAAGTCCCTTAAATTAAGTGA ATATTAGATTAAGGTGCCACTGCAGTGCTGGTATGGATGCTATTAATCCATCAGCATTAA TATTATTTTTAATCTAATCTAGAGATGCTGACATGGTTTATTTGACAGTAATTAAAAAGT
\end{abstract}

\title{
ALD26J13
}

TGGTACCCGCGGAATTCGGCCATTACGCGCCGGGGCCATTTTATTTAGACGCAAAACATT ACTGGGTAAATGATGACGTGGATATCGCTCGCTAGCAGCCTCCCACCGAGGACCTATCCA CGTAATCATTTACCCGAAAATGCCTTGTGTCGACACAAAACGGCCCCG

\begin{abstract}
ALD28M07
GTGTTGGTACCCGGGAATTCGGCCATTACGGCCGGGACGTGACTTGCAGAAACCGGAAGT AGGTTTCCGCGCTCCGCATTTTCGAAGTTTTCCCGTCCGGTTCGGCACATGGTTCCGATG AATGTCTCTTTAAAGCCGAGGGGACAAGAGGAGGGACATCTTCAGCTGAACATTAATCGG TCAAATGGACGCTGATCCCAGCGACGTCACGCCGACCTCGTCCAACAGGAAGTCCTCGGC CATCACAGGAAGTGCGAGAAAGATCAAAGACAACGCAGCCGACTGGCACAACCTGATGCT GAGGTGGGACAAGCTGAACGGGGAGGGGTTCAGCTCGGCGACAAACATCGTCAACATGAG GCGGTGAGGTTTTAAAAACATTCAGTTTTAGCTTTATAAATGAAAAGAGAAATAAATGTT TCACTTGCTGCATCAGTCAGACCCTCAGCTCTCAGGGAGGTGGTCTGGGTGAGTCCACCT CAGTTCCATCGGACCAGCTTCAGGCGGTCGAGGCGTCTCTGCAGGAAGAATGCTCCAGAC TCCAGGAAGTGGTCCAGAAAATGGTGAATCCTCGTCTGGTCTGAGGGTTTACTTCTGCTT CCTGGAGTTTCTGTTAGGAAACGTTTGTTTTCCTGCTCAGGCTGCTGTGCTGAAGAAGAT GGAGCGTCTGGT
\end{abstract}




\begin{abstract}
ALD35G19
GTTGGTACCCGGGAATTCGGCCATTACGGCCGGGGGGCGGAGGACGGGGAGAGAAGAGGA AGGAGGCTCCGGGAGCAAGCGAGACAAAAAAAGGGCCAAGCCTGGGACTTGGGGAAAAAC CGGGATTGAAAAGAAGAAGGATCATCACTTATTTTTTTTTCCATTTTAAATTTTATTTTT CCTGGAAACTTATATGACCAGACATCAAGCATAAGCCCATAAGACTTTCAATGATGCCGT CCAACACTGTCACCAACACCACTGCATTCAAAGGCTCAGACTTCGCTGATTCACCTGGAA ACAGAATGGGTGAGAGCACAATCCTGGACATGGAGCTGATGGAGGAGGAGAAGTTGACTT TAAACAGTGCCTCTTGTAAACCTGGAAAACCTGGGAGAAAGCGCAAGACATTTCCAGTGG AGAGCTGTGGCTCCCTCACAGAGAGTCTAAGTGTCGGCAACAGTTACTCCGGGGTGTGTA GGTCCTTCATGACCCAAGTCCACAATGGAGACATGATGCCCGATGCCTGCTTCCCAAAAC AGGAGAAGAGGGAAGTGGAGAACAGGA TCCCAAGAGACCAAGCAGAGGACAGCTCCCAGA GTTCGAGTCCAAGTCCTTCCAAAGAGAATGGCTTCCTGTCCGGCAGGGAGGATCAGGACT $\mathrm{T}$
\end{abstract}

\begin{abstract}
ALD08K10
TGTTGGTACCCGGGAATTCGGCCATTACGGCCGGGGAACGAACTGCCAACTCTGTTTAGA GGACGACTCCCCTAACCACTCTGCCACAGCCTCCCTTTGAGTTTGAGCGTGGAAAGTTGA TGTGGTAGTAACTGAGATTCATCCCTGAATAATTTAACCAAAATAGCTACATCTGTCTTT TCCCAACATGAGATGATCTTAATTTGAAACTCTGGCACAAATAGCAGCAAGCAATTATGA ATTCTTTCAAGAAATGTCTGTCCTTTCGTTTAAAATACCATTTGCATGAATTGCTACAAA TGTTTGAGACTAAAGTTATATTAGAACAGCAGCAATCTACTTTGGTCTTCAGTCCACTTA GCCTAGCTGTTAACTCAGCAATCTAGTCAGCATTAAACTATGTATTTTTTTATATTATAG ACATACAATATATACAACGATAAACTATATTTTTCTCCAATCTGAGACCATTCAAAAAGT TATCTACAACAGGTAAGACACTAATTGTTCCTGAACCTGATTTAATTTAAAAAAAAAACC TCTCCCTTTAAGTCCAAGCAAAGTCTCAGCCTGATTTCACGCAGTGCCCACGTGACTCTG TTCTTTCAGGAGCCTCGGGGTCCCAAATGCCATCGTAGTGACTAAGTGTGTGAAAGCCAA CCCGGACCGTCATGTGAC
\end{abstract}

\begin{abstract}
ALD09K02
GGGAATTCGGCCATTATGCGCCGGTGAGTGTATATCAAGGCTGCACAAACCTTTTCCACT AAAAGAAAATATATCTGAAGGGCTGCAGCCAAATACAAATACAAATGAAAATTCAAATAC TTCTTAAGTACAGTACTTTTATTTTAATGATTATGTCCTGCTGGAGTGTGACATGATTTG TTTTTGTACAAACTAATATGGTACCAGAATTTTATGTATTTATACTTCTCATTCAACCAC AATTAAATTCACTCTATCTAGGAGGCACAATTTATATGGATAGTTTTTATTTTTTACAAT AACAAGCTGGAATGAAAAAAAAGGAAAAATGAAATAAAAGTGACAATTTTCTACTTCTTG CCTTTTCAAAAATGTAAGGAGTAAAAAGTACAGATAATTGCGTGAAAATGTAAGAAGTAG AAGTAAAAAGTAGGCTGAAAAGTCATTACTCAAGTAAAGTACAGATACCCAGAAATTATA CTTAAGTACAGTAACAAAGTATTTGTACTTAGTTACTTTACAACTCTGATCATCACATTA TCAATCCACTCCAGATGTTTTAACCCTTTGTCTGGTGACCCACTGTCCTGCTTGTTTTGG ATCCCTGCTTTAACACACCTGACTGAACTGAATGAGCGGTCAAGCTTCTGCTAAACTTGA TGACATCATGAGTAGCTCATTTTATTATTTGATTCAGGCGTGCCGAAAGCAGGGAAACCC CTAAAACATGCAGGACAGTGGCATTTGAGGAACAAGGT
\end{abstract}

\title{
ALD26F21
}

GTTGGTACCCGGGAATTCGGCCATTACGGCCGGGGGAGGGTGTGGATCTACAATAGTCTG CTGCTGGAGACAACCACGGACTGTTTGACAAATACCTTCTCCTTTGGCTGTACTACAGTA 
AAAACCGCGGACATACCAGTGTTACTAGTGCCTTAGAGCGTCTCTGGTTGTGTCCCAGAT GAGGGACTGATGCTGGTCAGAACTTGCCTAAACTTGGTCCTGGTTTAGTTCTGCATCTGT TCTCACTGGTGCACCTTGAGAAAAGAAGAATACATCAGTCGAGATGGATGATATCTTCAC ACAGTGCCGGGAAGGCAACGCCGTGGCTGTTCGCTTGTGGCTGGACAATACAGAGAATGA CCTGAATCAGGGAGATGACCGTGGCTTCAGCCCGCTCCACTGGGCGTGTCGGGAGGGGCG GTCCAATGTGGTGGACATGCTCGTCATGAGAGGAGCACGCATCAACGTCATGAACCGGGG GGATGACACTCCTCTGCACCTGGCTGCGAGCCATGGACATCGGGATATTGTGGGGAAGAT GATCCAGTGCAAAGCAGACACCAATGCAGCCAACGAACATGGAAACACACCACTGCATTA TGCCTGCTTCTGGGGCCATGACCAAGTCGCTGAGGACCTCGTGAGTAGTGGGGCTCAGGT GAGCATCTGTAACAAATTTGGCGAAACTCCTTTGG

\begin{abstract}
ALD33D20
GTTTTGAGGAGCGCAAAAAAAAACCAAAGCAAAAACATGTTCAGTCTAGCAATGGCTGCA TAAATGTTAAAAAAGGTTAACAAAGGATATTTTTAACGCGAACACAGCGATAACGCGTTT ATTAGCCCCTCAAGAATGAGCAGGTGGGGGGACCGGCTGAAGAAGATTGAGCAGCTTGCT CAGTCATTCCAGCAGCATCCCCTGACGACGCGCTACAAGCCTCGCCTTTGGCCATGCCAG CCCTCCTCTGTCTGGAAGCTCTTTCCTCGTCAAAACA TGGCCATCAGTTTCACTCAAAGC TGCAAAGAGGCTGTGCACGTTTTTGCTCTTGAAAAAGAAAAGGCGTCGCTGGGCCAAAGG ATCTACCTGGTTACCAGCTACAGCGAGCTGTGGCATTACTACAGAACTTATCCTCAGTCC TTAATGCACTGTTATGAGGTGATCCCAGAGGGGGCTGTGTGTAAGATTTACTTTGACTTG GAGTTTCACAAACCTTCAAACAGAGGATCTGATGGGAAGTCTATGGTGTCTTTGCTTATT CAGTATGTTTGTGACAAGTTGATGGAAGTCTATGGGATTAAATGTTCGGCAAAAAATATC CTCAACCTTGACTCCAGCACAGAGGAGAAGTTCAGTCGACATCTCATCTTCATCCTGCAG AACGCTGC
\end{abstract}

\begin{abstract}
ALD37M09
TGTGTTGGTACCCGGGAATTCGGCCATTACGGCCGGGGCAGCGAGTCGTAAACTCAGACT TTATTTTTTGTTAGTTTTGTTTTTAGAGGCGATAAAATCGAATCTATTTTTGCTGTTTTC ATCAGTTTTTTCTTTCCTTCACTCGTTTTTATTTTCACAGCTGCTCGTTTCTCTGGAACC GTATAAGAAGGAAACAGTCGTCAGTTTTCTAAGTTTAACTCCAGAGCAGCTCTGACCTGA TAAGACCTTCATCCTGTTTTTGGCCTCAGTTTGACGTTTCTGTGATCGTCTCTGCAGCCT GACAGGAACGAGGAAGTTGTTCAGTTTCCTGTTGAAGGCGTCTGACTGGAGATGACTGGA AACTGGGGGAAAAAATCTTAGTTTTAGTTCAGTTTAAGCTGCAGTGGAGTCAGATGTTTG TCTCTGGTGACCTCTGGCCTCTCTGTGTTTCCAGTTTGCATCAGTTTCAAAATAAAAGCG TTAAACTGCAGGTCTCATCGTTGCCGGCGGCCTGCAGTAACCGTGCAGCAGAGCGAGCTC GTGTCCTGCTGGAGGTTTGACGGCGATGCAACACGTCCTCGTCCTCGACAGCTCCTCCAC AGCGAGCGCCATCTGTCACATCGCCTCATCTTCCAGGAAATTACTGCTCGGACCGAAAGC CTCAACATTATCTGATACGTACCCCGGCCGGGCCCGAATGAACTC
\end{abstract}

\title{
ALD35I20
}

GTGTTGGTACCCGGGAATTCGGCCATTACGGCCGGGGATGGCT

\begin{abstract}
ALD02J22
GTTGGTACCCGGGAATTCGGCCATTACGGCCGGGGACAGTGCCCAGGACAACGGCCTCAG GATGACCGCCCGTCTGAGAGAACTGGAGGTGAAGGACCTGCTCTCCCTCTCCAGGTTCTT CGGTTTCTGCTCGGAGACCTTCTCGCTTGCCGTCAGCTTGCTGGATCGCTTCCTCTCTGT
\end{abstract}


GATGAAGATTCAGCCCAAGCACCTGTCCTGCGTCGGCTTGTGCTGCTTCTACATCGCTGT GAAAGCCTCGGAGGAGGAGGAGAACGTGCCGCTGGCCAACGACCTGATCCGCATCAGTCA GAACCGCTTCACAGTGTCCGACATGATCAGGATGGAAAA GATCATCATGGAGAAGCTCCA CTGGAAGGTGAAGGCCCCCACGGCTCTGCACTTCCTCCGCCTGTTTCACGGTCACGTCCA GGAGCAGCTCGATGCTGAGAGCAGGAATATTCTGAGCCTTGACAGACTGGAGGCGCAGCT GAAAGCATGTCACTGCTCGTTTGTCTTCTCCAAAATCAAGCCCTCTCTTCTTGCCATTGC TCTGCTGTGCTTTGAGGCCCAGGAACAACACGACCCTGAGGACGCAAACCAAACATCCGA GGCCCTGAGAAACCTCCAGCAGCTGCTGAATGTAAAAGATGGTGACCTGGTGTGTGTGAG GG 


\title{
Appendix $\mathrm{C}$
}

\section{Table 3 sequences}

\begin{abstract}
ALD23D03
TTGTGTTGGTACCCGGGAATTCGGCCATTACGGCCGGGGAGAAAAGTCACTCTAGTTGTG CAGGTTCCTCCCTCCAAAACCAAGTGCTGGGTTGAAGGCAGCGAGGAGAAAGGCGGCTCC GTCTCCCTGCGCTGTAAATCCTACCAGGGAACTATTCCTCTCACTTACGTTTGGACGAGG GAGACCGGCGGTACGATGCCGACCACTGCGACCCAAGACCCCAATACTGGAGAGCTTCTG ATAAAGAACCATACAGACAGCAACACTGGAGTTTACACATGTGTGGCTAAAAATTCAGTC GGTCAAGACCAGTGCAAATACACACTGGGTGCATACAACCCAACCAACAAAGCGGGCATC ATCGCCGGTGCGGTGATAGGCGCTCTGCTGCTGTTCCTCCTCCTCCTGCTTCTCATCTGG TGCCTGATCTCCTACTGCAACAAGAAGCGCTACCAGAAGGAGGTTTCAAATGAAATTAGG CACGACGCTCCTGCTCCAGAGAGCAAACCGCCCAGCCGAGCATCGAGTGTTCGATCCATG GTGGGGTACCGCACGCACCACGGGGTCCAGTACAACGCCGTGGCGAGCCCCCTGCCCAGT GTCACTGAATCTGCCCCCATCGTGGCAGATGGCAACAACGACATGCCCTCTGAAGGGGCT AGAGGGGCTTCCTTCAGATACGACCCTCAGTACGGATACGCTGTGTAACCGCGAGCCTGA AAACAAAAGATCTTATGTAAGAGACTTAAAGTGC
\end{abstract}

\begin{abstract}
ALD37I12
TGGTACCCGGGAATTCGGCCATTAAGGCCGGGAGCATTCTAAACAACAACAAACTACTGC TGCTGATGGAAAACAAGTCAACATCAGCTGTTATAAATTGGTATACATGGTTTAAAGCAA TAGCTCAGATCATTTGAAGTGCTACCAAAGGCTTATCAAAAGTTAATAAACTACTTGTAG AAAACTTTCTGACTGGATTAACTTGCCCATTTGACTGTGGCCAATACCAAAGTCAATTGC TGCATTCTTAAAACTACAATTTCAAAAAGTTTGCAGCAGTTTGTGTGAGTACTATAATTC AAAGAGCTACCTGAACCTGGTCAGACTCAACTGTTTATAACCCTTTCTTCAAAAGATCTG AGCTATCCCCATTAGTTTTACAAAAAGTCAGAAGTTGTGGTTTTTCACATGTGATAATCA TTATTTCTCTCTTATCCTTGTATCACTTAGGGGAGTTAAAGGGTGGTTCTAGGTTTTAAA AAAAGGAATTTTAAAGATTTTTCTATCAGATTGTTGTCAAAGAAACAAAATATCCAACTC AAAATTTTTTTGTTCTTTAGCTTTTTTATTTAAAAGCAAACATACAGTACATAGTTTATG GTGAATATACTAAACACTGTTGTGTTCAAAATACTACTTATTACCATTTACTTTGCTGCT AACACATTTAAGTGAGTGACAATAATAATGGGACATTGTAGCTATGCTTTATTGTCTGCG TTTGTGTGAGATTTTT
\end{abstract}

\begin{abstract}
ALD16D08
GTTGGTACCCGGGAATTCGGCCATTACGGCCGGGGGCGTTTCTGCTGTCGTTTGAGCAGC TCTTCCTTTAGTCAAGAATGCAGACCAGGGTTTCTGGAGAACATGGATTTTCCTGGATCA GATCTGACGTTTCTGGCCTCTCCTTCTGCTGAGCACTGTCAGAAGCTGTGCACCCAGCAC CCCTCGTGCCTCTTCTTCACCTTCACACGGTCCGACTGGACCAGATACAACACCTTTTTT CGCTGCTACCTGAAATCCACTTCATCTGGAGAGCCAGCCTCACAAACTCCTCTTCAGGGC GCCACTTCTGGATTCTCTCTCAAAGCCTGCAGTCAAGACTTACAACCGTGTTTTTCGGAG ACTTTTGAGAACGTGGACTTCCCAGGGGCCGACTACAGAGCCCTGTTTACACCCGACTAT GAGGAGTGTCAGAGAGCCTGCACCCAGGACCCTGGCTGCCAGTTCTTCACCTTTTTAAAA GAGGGTTTTTCAGCAACAAGTTACAGGTTCAAGTGTCATCTGAAGTTCAGCTGGAGTTTA CCGGTGATCCCTGACATGGAAAAAAAGAGCTGGACCTGTATCTGGGTTTTCCCAAAGAGC ACAAATATCTCGTCAACAGCTTGATACAGCATG
\end{abstract}




\begin{abstract}
ALD32D03
CCGGGAATTCGGCCATTACGGCCGGGGCTTTTTTTGAGCTTTGCTCAGCTCCAACATGGC GGACACCGGTGTGGTGGAGACGCTTCTGAAGCGGCTTGAGAAAGTGGACGGCGGCGTAGA CAGCGTGGAAGTGGCCAACAGCCTCGGAGTGGACCACCAGCACATCGTGGGAGCTGTGAA GAGTCCGCAAACCCTCGGTGACGTGATTTCAGCGGATCTGCGCTCCTCCAAACACTGGGA GCTGACGGAGGAGGGCACGGAGATAGCCGAGCAGGGCAGCCATGAAGCCCGGGTCTTCAG CTCCATCCCTGTGGAGGGTTTACCACAGGCTGAGCTCATGAAACTTTCCTTCGGAAAGAT CGGATTCAGCAAGGCCATGTCCAACAAATGGATTCGAGTGGACAAGTCCCACGAGGGAGG CCCCAGGATATTCAGAACTGTGGACAGCATACAAGACCAGGTGAGAGATAAGCTGCTCGT AGTAAAGAAGGGTGACTGTACTCAGCTGGAGGAGAAAGAGAAGAGTGAACTGAAGAAGAG AAAACTCCTCTCTGAAGTGACGCTCAAGTCTTATTGGATCACTAAAGGCAGCTCTTTCAG CACTACTGTGTCCAAACAGGAGACGGAGCTCACCTCTGAAATGATAGCTACAGGCAGCTG GAAAGAGAAGAAGTTCAAACCCTACAATTTTGAGGCCATGGGCATAGCCCCAGACTGTGG TCACCTG
\end{abstract}

\begin{abstract}
ALD05E02
CGGGTTAACCGACCTGACGGTCCGGTTCAAGGCGCCCGTGGCCGACGTCTACACACC CCGGATGCTTGCTTTAATAAATGGCTCCTCCTGTTAAATATAATTTTAAAATTTACA AGATAAACATCCTACGGTATTTTTGCAGGCTTTATTTTACAGTTAAAATAGATGTTT ATGAATTAAAGTCTAATGTTACAACTAACCTGGGATGGCCTTTAAGGAATGAAACAC AGGATGTAGAAGCATCATGGAATACAGAGGCCATCGTTTTGTGTTTTGACATGACTT TTTGTTTGCTTTGTCCGGCGCATTCCAGCTGTTTGTCGAGTAATCACAGCAGTCCTG TTTTTGTTTCATTCAAAGACTTTTTAGGTCGAAAAACTCCACAAACTAATGTCCGTT ATTACTGTGCCGTCTTTCTGATTCGATCGCTGCTCGGTGGTTTCTAGTTGTGTTGGA CCACTTGAATATGTGTTAACCTCAGGTTGTTTTTGTTCCAGTGTTGAATTTTATTTA TTTTTTTTTTTTATCATTTCTCATTGTGCTGGCCTTTATGCAGCCCCCCCAACAATT GAAGAAATACTAAAACGTCTCCCCCTTTAGGAGAGAAATGACGCTACAAGATGTCAG ATGGGGGTGAAAACACCAAAGCAGGAGGTAAAAACATTTCAGAAGTGGGAACGTACA CTACCTTATGCTAACATGAAGAGCAAATAATTATATTGTTTGTTTTTGCATAAAAAT CCTTTTTACTGTAGCAAC
\end{abstract}

\begin{abstract}
ALD36C23
GTTGGTACCCGGGAATTCGGCCATTACGGCCGGAGCTGATTGTTTTGTGATTTTATAGCA CTTTACCTTCAGAGGGCTGGTTTCTTACGTCCGAAGCAGTATTCCAGACGAGCTGTGACA AGTTTTCATATTGTTTGTTTTTTGATCCTTTTCTCCGATACATTTATAGTGATTTACCTC AGATAGAAAAAAAAACAACAAACTGCAGGATCTTCGTTTGTTGAACAAACAACGAGACGA GGCGAGTCTGTTTAGACGCTTGATACAACCCCGAGCAGCAAACATCCATCAGATATATGA TTTTTTTTAGTCAAATAAAGTGTTTTCCTGCGCTTTACTTCCCTATTAACCTCACATTTA AACAATTCATTCTCTCATCATGAACGACAGGCTTATCGGGATGAATGAAACTGTAATTCA GGCTTAGAGTTTCCTGCATGAGCTGCAGGTCGGATCAAGCTTGACGCTGTGAAGCTTTAA AAGCTGGTTTTCTCTGGTTCAAACCTGTGTTTTCCCTCATGAATCCGTTCAGGAGCGGAA AAACTTTTGCCTTAACAGAACTCTTATCGGAGCGGGAGCTTTTAGGAATTAATCCTGGGA TTGTGCATTTGAATCTGAACTGTTAGGCTCTGGCTTGTTTTAAAAGTAGATCCTTTGTGT TTCTGTAGTTTTCTAGTTTTAACCTTGTTAGAAATGTAACTAACATCCAGCCTGCTTTAA CAATAC
\end{abstract}




\begin{abstract}
ALD40F05
GAATTCGGCCATTACGCGCCGGGTAAAGGTTAACTACAAAGCCAAGGCAAACCTCAGAGA CTGAACACTCAGGGGGATTTCCTCATTCCTTAGCTCTGGGCTCAGATTATTCACATAGTT TGGAGGCAAAGTGGAGCGCCAGAATCCACTGAAACTTGTTTGGAATCACAGATGGAAGAA TGAAGTGCAGATTAAATGATTTATGGAAATATCATTTGGAACAGTGTCCCTGTGTGTTGA TATGCCAAACTCACAGCTGACATGTGTTAATGTACATAGGCGTTAATTAGAGTCAGTGTT ATTGCAAACCATTATTAATGTACATGAGTGTTAGGGGTTGCAGTAGCATGGTGGTTTTGC TGCAGTCTCATAATCCTGAGAATGCAAGTTCATGTTTAGATTGAGTCAGAAAGGACATCT GGTGAAAAACAATTGCCAAAAGTTTCATCTAAGTTGCTAATTGGGGCAGCCTCTCTCCAC AAAACCTAAGTGTTATTATGCAGCCTTGTTAATGTAATTTGTAACAGTTTTGGACACATG CTAAAAACGTTCAGTTTTTCATGTAAGAAAAATCTTATCTTTCAATCTGCCCACAAATTC ATAATACTGAGCCAATCCTGAAGCGGCAGATCCCACAGAGGACAGGGCCATGAGGAAATG TAGGTCAGATCCGGTTCTTTCAGAGTCCAAACAATCCC
\end{abstract}

\begin{abstract}
ALD28M24
GTTGGTACCCGGGAATTCGGCCATTACGGCCGGGGAGGAGGCCATCGCATGGCTCTGCGG GAGCCAGCCCCATTTGCCAAACCAACAGGTTGTGAGGCAGTTGTCCCACACGACGTGGTG ACCATTTCTCAAGCACGAAAGGGAACGCCAGCTCACCGTCCAGTTCGAGTCTATGCTGAT GGGATCTTTGATCTTTTCCACTCGGGACACGCTCGAGCTCTGATGCAGGCCAAAAATGTC TTCCCCAACACTCACCTGATAGTAGGAGTGTGCAGTGATGAACTCACCCACAAGTTTAAG GGCTACACGGTGATGACGGAGGATGAACGCTACGAAGCGTTGCGGCACTGTCGTTACGTC GACGAGGTGGTGCGAGACGCTCCCTGGTCACTTACCACAGAGTTCCTCAAGAAACACAAG ATCAAATTTGTGGCTCATGATGACATTTCGTACACTTCTGCTGGATCAGAGGATGTTTAT AAGCACATCAAAGAAGCAGGGATGTTTGTGGCCACTCAGAGGACAGAGGGTATCTCCACA TCAGATCTGATTACACGAATCGTTCGAGACTACGACATCTATGTCCGTCGCAACCTGCAG CGAGGGTACACGGCTCGAGAACTAAACGTTGGGTTCATTAATG
\end{abstract}

\begin{abstract}
ALD33H14
TTTGGAGATGATGAATTTGCTTTTGGCTACGAGGTCTCCAAATCCCACTTTTCTCCCCTG CTGGAAACCTGATGCACCCTCTTCGAAGTCAGAGATTCCTCTAAAGTCACTGAAATGTTT GACTGTGTGGAGCCTGATGATGTTGAAAGCAACGTTCCGGAGATAATTACTGCTGGTTTC
\end{abstract}

\begin{abstract}
ALD39L22
GTTGGTACCCGGGAATTCGGCCATTACGGCCGGGGGCACGAGGAGACGGACGCACATCCA GATAGAAGATGAGTTTTCCAGCTCTGGCCTCTCTCCTGCTGGTCATTTCTTCTGTCCAGG CCTTCCAGTACACGGACCTGGTGCAGTACGTAGACAGCCACCAAGAACAATATGTGCAGA CCCTCAGGGACTGGGTTGAGATTGAAAGCGACTCCAGCAACATCCTGAGAAGACCTGACC TCCATCGTATGATGGAGGTGGTGGCTCAGAAGCTGCGGCTCATGGGAGGAACCGTGCAGC TCGTCGACATCGGAGAGCAGGAGCTTCCTGACAATCAGACGGTGGAGTTGCCTAAAGTTG TGACGGCTGAGTTTGGTAATGACACCAACAAACACACTGTGTGTGTCTACGGACACGTTG ACGTCCAGCCGGCCAAACTGGAGGACGGCTGGGCTACAGAGCCCTACAACCTGACAGAGA TCAACGGAAATCTGTATGGAAGAGGAGCTTCAGACAACAAAGCTCCCGTTCTGGCCTGGA TTCATGCTGTGGAGGCTTATCAAGCCCTCCAGATGGAGTTACCAGTAAATGTGAAGTTTG
\end{abstract}


TCATCGAGGGGATGGAGGAGACGGGGTCTAACGGTCTGGATGCCATGATCCTGGCCCAGA GAGACACGTTCTTCTCAGATGTGGATTACATCATCATTTCTGACTGTGGCTGGAGGAGCA GACGGCCGGCCCTCACGTATGGCACCAGAGGAAACTGCTACTTCTTTGC

\begin{abstract}
ALD24L13
GTTGGTACCCGGGAATTCGGCCATTACGGCCGGGGAGAAGGAGCTGTTCCTACCGGCCAT GCGGAAGAAGAGCAGCTCCAACACTGCTGAGGGCTCCGTCAACGGTGACACACTGACCGC CCACTGGTTAGTGGACGACATGCTCACTTTTGAGAACGTGGGTTTCACAAAGGATGTGGG GAAAATCAAGTACCTCATCTGTGCAGATTGTGAAATTGGACCAATCGGCTGGCACTGTTT GGATGACAAAAAGAAATTCTATGTGGCTTTGGATAGGGTGAACCATGCATAATGTTTTCA TGCATATGTGAGCCCACACGGACCTCATTAAATGTTTTGGCAGGCTATGCATTGTTTTTA TGGATTTATGCCCAGTTTACCCCGAATAAGTCCATCGTACACACACTCACTGATACTGTA CTGTTAAAGGGATTGTGCCATGTTCATTTCCATCGATCATAATGGCATTAGTACAACCAT TCTGTTCTAATAAAGTTATTAATGAAGTCATTATTTGAATGTCATACTTGTTATATTTGT TACCGTCACCTAGATGAAACAGTTTAGAACAATTTACAATAAGTCCCTTAAATTAAGTGA ATATTAGATTAAGGTGCCACTGCAGTGCTGGTATGGATGCTATTAATCCATCAGCATTAA TATTATTTTTAATCTAATCTAGAGATGCTGACATGGTTTATTTGACAGTAATTAAAAAGT
\end{abstract}

\title{
ALD04P15
}

GTTGGTACCCGGGAATTCGGCCATTACGGCCGGGGGGAAGAGGAGATGTCACCGTCGGCG GTACAGATAGATCAGTTCATAGCATCACTCAGTTCTGTTCAAAGAAAACTCGGATATATT TGTTAGCCACACTGTGCTTGTGTTTAACATGTTGTAGCAGCGCTCCCAGTGACCAGAGCT CTCCGTCTCCAGACCGGCCGTGACAGGACAGCAGCACACAGATCAGGCCTGTTATGGCTG CCCGGCATCAGGGACCTGCAGCTGCTTCACACGGCTCAGCCGAGGCCAAAAAGACAGACC TGAGGACCAAAGAGAAGAAGTGCTCTTGGGCCTCGTACATGACCAATTCTCCCACCATGA TAGTGATGATTGGGTTGCCCGCTCGAGGGAAAACCTACATGTCCAAGAAGCTGACACGTT ACCTTAACTGGATCGGAGTGCCGACAAAGGTCTTTAATCTTGGCGAGTACCGGAGGGAAG CGGTGAAGTCGTACGAGTCCTACGATTTCTTCAGACACGACAACAAAGAAGCAATGAAAA TTAGAAAAGAGTGTGCTCTAGTAGCACTGAAGGATGTAAAGGTTTACCTGAGTGAAGAGG GAGGACAAATTGCTGTTTTTGATGCCACAAACACCACGCGAGAGAGGCGAAACCTCATCC TTAACTTTGCACAGGAAAACGCCTACAAGGTGTTTTTTGTTGAATCAGTATGTGATGATC

\begin{abstract}
ALD34H19
GTTGGTACCCGGGAATTCGGCCATTACGGCCGGGGGAAAGGGTTTATTGTTTCTTTGCTT TTCGGGAGCTGAATTTGTTTGCGATCCGGTCAGTTCTACAATCAAATAACGAGACGGAAA AATGGCTGGTGGCAAGGCAGGAAAAGACAGCGGGAAAGCCAAGGCGAAGGCGGTGTCGCG CTCCCAGAGGGCCGGGCTGCAGTTCCCGGTGGGTCGTATCCACAGACACTTGAAGACTCG TACCACCAGCCACGGTCGCGTCGGAGCGACGGCAGCGGTTTACAGCGCTGCCATCCTCGA GTACCTCACCGCCGAAGTACTAGAGTTGGCAGGAAACGCCTCCAAAGACTTGAAGGTGAA GCGTATCACTCCCCGTCACTTGCAGCTGGCAATCCGTGGTGATGAGGAGTTGGATTCTCT CATTAAGGCAACGATTGCCGGCGGAGGTGTCATCCCCCACATCCACAAATCCCTCATCGG GAAGAAGGGCCAGCAGAAAACTGCATAAACGCCACGTTGACCACAGATTTTGGGGCTTGG GCTTTGTTCGGACCAGGAGTTCACATTTGTTCTTTTCTATTATTAATGTTATAGCAAACG AAGAGTGATTGTTAGCCTTTGTGTTGTTATATTTTTCCCGTAATTAGAGGAAAGTACAGC TAATCCCTTAACAGCAGAAAAACCCTTTTTTATGTCCTTGTAGAGCGTTTGACTGAGGAG CTCATTATTACTGAAATGATCAGTGAATTTGTTTGAT
\end{abstract}




\begin{abstract}
ALD32F04
GTTGGTACCCGGGAATTCGGCCATTACGGCCGCGGGAGGACAACAACACACAACTCTGCG CAACACTGTGAGCAAAAATGGCGGACGTAAACCAAATGGAGAAGAAACTGGCTGAATACA AGTGTGACACCAATGAAGCAGTCTGCCTGAAGCTAGTTCGTTTTCCTGAGGACATTGATG ACGACAGCACCACATTCCACCCAGAGTACAGTCACCAACAGTTTGGAGATGATGAAGTTG CTTTTGGCTACAAAGGTCTCCAGATCCAACTTTTCTACACTGCTGGAAACCTGGGCACCC TCTTCAAAGTCAAATATTCCTCAAAAGTCACTGAAATGTTTGACTGTGTGGAGCCTGATG ATGTTGAAGGGAAGGTTCGGGAGATAATTCCTGCTGGTTTCACCTCCAACACGGACGACT TTATCTCCCTGCTGGAGAAGGAGGCCAATTTCAAGCCCTTTGGCACCATGTTGCACTCTT ACACCATCCACAACGAGGAGGCGGGAGAGCTCACCTACCAGGTTCACAAGGCCGACATCA CCTGCCCCGGATTCCAAGAGTACCACGAGCGCCTGCAGACCTTCCTCATGTGGTTCATCG AGACTGCCAGTTTCGTTGACACAGACGACGATCGTTGGGACTTCTTTCTTGTATTTGAAA AGTACAATAAAGACGGGGAGACTCTCCACGCAACTGTTGGCTACATGACAGTTTATAATT ACTACGTATACC
\end{abstract}

\begin{abstract}
ALD33D11
GTTGGTACCCGGGAATTCGGCCATTACGGCCGGGCGCCGACGGCGGATGCTGTGTTCCAT TCTGGAATAACTAGATGTGAACTTCAACATTTGGGGTTTAATTTTTACATTTGGATTTAA AAATTTAGATTTTGAATTTAACATTTAAATGTAACATTTACTAAAGATTAGCTTAATGTA AGAACGGTGAGGTAAACAATGAAAAGGATGGTAGTGAAAATTTTTAACATGTATTCTGAT TTTGTATAGTTTAGTAGGTAAACATATTGTTGTTTTAGTCTTTATGAAATTGTGACTTAG AATAGGGAAAAAAATAATTTATTATTTTTTAATAAGCTTTGGAGAAGGGTGGGATTAAAT ATGTTTATTTTACTTCTTCCCATTCCTTTTCAAATACGTTGATTTTGTTATAACTTTTTA TCATTTTTTTGTTTTGTTCTCTATACATGTTTGAAATAAACTAACATTTTATTTTACACT TGGTGCCTCATAAAGATCAGCTGATGCTTGTTGGATTCATGCAGCAAAGTGCTGAGTCAT CCCTCTGTTTCAGGTGGTTTCAGCAGGGACAGAACCCTTACACCCGCTCTCAGGACTGGC TTTTCTCTGGAGGGTATTGGGACCGAACATACAGCCAGCTGCC
\end{abstract}

\begin{abstract}
ALD15H03
GTTGGTACCCGGGAATTCGGCCATTACGGCCGGGGGGGTTACCGACGTGCTCTTGGAAGC TGAAGGCGTGCTGAGCTTTCTCCCGAGTTCTTTCCTTTGTCAGTCTTGGAAGCTTTTTCT GCCGCTGCATTTGTCTGAACTCAGCTGCCTGCAGCACAGATAAAAATGTTGAGAAGTTCT TTAACTCAGGTTTTCAGACAGGTGGTGAGAAACTGGAGTACAGACACAAGCCTGATCCTA GAAAGATCGATAAACCGGGTGCAGCTGTTGGGCCGCGTTGGTCAGGACCCGGTAATGAGA CAACCGGACGGTCGAAACCCCGTCACCATTTTCTCAATGGCAACAAATGAAATGTGGCGC ACTGGAGACGGACAGGCGGGCTCAACAGGTGACGTCAGTCAGAAGACGATGTGGCATCGG GTGTCCGTGTTCAAACCAGGTCTGAGAGACGTCGCCTATCAGCACGTTAAGAGAGGAGCC AGGGTTCTAGTAGAAGGAAAACTGGATTACGGAGAGTATGTGGATAAAAACCAAGTCAGA CGCCAGGCCACGACCATTATTGCAGAATCNATCGTGTTTCTGAGCGACAACAGAGACAAA GAATGAGGGCCTTTCTGTCCGTCCACATGATAACTTCTAGTTCTTTGTACCAAAATAAGA CTCGAGAATGATTGATTCCTGAGGAACAAAATAAGATCACGTTTGACTGT
\end{abstract}

\title{
ALD15L11
}

GTTGGTACCCGGGAATTCGGCCATTACGGCCGGGGGTTCATTTGTGGAGTGCTGCTGAAG AGTGCGTACAAGTATTTTTTTTGGTGTCCGACTAAGTTGTTTCTTTTTAATTTTTGATAA AAATGAGAGAGATTGTTCATTTGCAGGCTGGCCAATGCGGCAACCAAATCGGAGCGAAGT 
TTTGGGAGGTGATAAGCGACGAACACGGCATCGACCCGACCGGGACCTACCATGGGGACA GTGACCTGCAGCTGGAGCGGATCAACGTGTATTACAACGAGGCCTCAGGAGGCAAGTATG TTCCTCGTGCAGTTCTGGTGGACCTGGAGCCTGGCACTATGGACTCTGTGAGGTCTGGTC CTTTCGGTCAGGTGTTCAGACCTGACAACTTTGTCTTTGGCCAGAGTGGAGCTGGTAATA ACTGGGCTAAAGGCCACTACACTGAGGGAGCTGAGCTGGTGGACTCTGTTCTGGATGTGG TGAGAAAAGAGGCCGAGAGCTGCGACTGCCTCCAGGGTTTCCAGCTCACGCACTCTCTGG GAGGAGGCACGGATTCTGGCATGGGTACGCTCCTCATCAGTAAGATCCGAGAGGAGTACC CGGATCGCATCATGAACACTTTCAGTGTTGTGCCCTCACCTAAGGTGTCAGACACGGTGG TGGAGCCCTACAACGCCACGCTCTCTGTCCACCAGCTGGTGGAGAACACAGATGAGACCT ACTGCATTGATAATGAGGCCCCGTATGACATCTGCTTCCGTACTCTGAAACT

\begin{abstract}
ALD28D03
GTTGGTACCCGGGAATTCGGCCATTACGGCCGGGGCAACACCCAAAAGGTGATTATTTTA TTAGCTATATAAACATACACTATGATCAAAATGTATGTAAAAAAAACAAAAGTTGTGAAC CATCGTACTTCCTTCATCCACTGACTGAAAACTTTCACAGCTTCTTATTGAATCAGAAAC TTAATGCTGTTTAAAAGGTCACGTTACTCATTTTATTTCCACTTTTGACTTGACTCAAAT CTGCGTTTGGTACGAAGACTGCAAACCAATGTCACCAAAACGACTTTGATTTCAAAATTT TCATTATTTTTTTTTCTAACACAGATAACATTGGTTTCTGCATTTCTTCAAATATTTTTC ATGAATTCTTTTTCGTCAGGCTTGAGTCAGTGTCCTTTTTGTGTTGCTTTTGGTGCAGTT TCTGTTTATTGAAATGACACTTTGAAAACAACTCTATATTTTTTTTTTTTTCAAATGCAA ACGACCTATTTACTGTGGCTGGAATAAACAGGAAGCTGTGGTCACCGACACATTGTGACT CGTCTTTTTACCTGATATTTTTGTGAACTCTCGGTTCTTCGGCGCCTCCCACCAGTGCTG ATTCACTTTCTTCTGAAGGGCTT
\end{abstract}

\begin{abstract}
ALD29I17
GTTGGTACCCGGGAATTCGGCCATTACGGCCGGGCAGCCTGGTGTAGCAACTCTCTGCCC CTCTGTAATAAATGTCTTCACACAGCGCACACAAGGAGCTCTGTTCAGCTGCATTCAGGA CTGTTCTTTCTACATTACCCAAGTTTATCATATTCTTCCTCTCTGCGGAGTTCTGATCTC ATTCAAGGGGCATAACCTGTTAGGGTCTGGTGCACTCATAGGGATTGTAAAATCAAGGTG CACAGATGATCAGACTTGTATGTTAAAAGCAAACTGGAAGGATTTTGATTTTGTTGTTGA TTCTGTTAAAAATGTTGTATATTTACTGGTTCAGCAGAGCATCAGTAGGCAGTAAAACTT CTGATTTACAGTGTAAAGATCTTTATATAGACATTTGAAATAGACATTTGAAATAAGGCA TTATTGGTGACTACAGACAATATTTTCAAACCGATGCCAAATCGCTCAACCTTTGGTGTG ATTTTAATCAAACTGAAATTAGTGCACTTTAAAAAACGTGATGCTTTAATTAATATTTCT TAAATTAGAACAAAACCAACCACCTCAGCCAGTTATGAAATTATTAACCATTTGTTGTAT CTGTGCTGTAAAAACAGAAAACATGCACAGATCGTATTATTCAGGAATTAGTAAAATAAA TTGAGTTTGTTTAATGTTTAAACCCTAATAACCTGAAATAAGCTTTCAACCAAGTATGAT TTTGTTTTTAATGAAAGTGCTGG
\end{abstract}

\title{
ALD34N22
}

GTTGGTACCCGGGAATTCGGCCATTACGGCCGGGGCTTCTTTCCAGACTCAGACTAAGAA GCACACGAGCTTCATCATGGTGTACAAAGTAGCAGTGATCGGGGCGGGACCCTCAGGTCT GACCAGCATTAAAGCCTGTCTGGATGAGGGCTTGGAGCCGACCTGCTTTGAAAGCAGTGA TGACATGGGTGGACTGTGGAAGTTCAAGGAAGTATCAGAGCCCAACAGGGCCAGCATCTA CCGCTCCCTAACTATCAATATCTGGAAGGAGATGATGTGCTACAGTGACTTCCCCATCCC AGCTGATTACCCCAACTACATGCACCACTCTAAAATCCTAAAATACTTCAGGATGTATGC AGATCACTTCAAGCTACTTTCTCACATACGCTTTCAGACCTCAGTGAAGAGCGTCACCCA 
GAGACCAGACTTTTCTTACACCGGTCAGTGGGAGGTGGTCACAGAGGATAAAGATGGACT AGAGGAGAGGCATGTCTTCGATGCAGTAATTTGCTGCTCCGGTCACTTCAACTATCCCAA CCTGCCACTCAAAGACTTCCCAGGAATTGAGACATTTGAAGGAACATACTTCCACAGTTG GGACTACAAGGGACCAGAGGATATGTATGGTAAGAGAGTGGTGGTCATCGGCATCGGTAA CTCCGGAGGGGACATCGCTGTGGAGGGCAGCAGAGTTGCAGAGCAGGTATATCTGAGCAC TCGCAGTGGTGCCTGGGTCATCCGTCAAGTTTCTGA

\begin{abstract}
ALD19B23
TGTGTTGGTACCCGGGAATTCGGCCATTACGGCCGGGGGCAGCAAAGAGGACAGTGAAGG CTTTGAAAGATGTGTCGTATCTTTGCAAGTTGTGCTCTGGCAGCCCTGCTGCTGGCTGTA GTTTGGGCAGATCACCATCACCACCATGATTCCAGTCACAGCCATGAGGGTGAAATGGCC TGCCATGTTCTCTCCCCTCATAATGCAGACTTTGCCTTCACCCTCTATAAACATCTAAAT GCCAAGGTTGCTGCTGGACAGAACATCTTCTACTCACCGCTGGGTATCTCCACTGCTTTG TCCATGCTGTCTGTAGGGGCCCGTGGTGACACGCACACACAGCTGTTCTCCAGCTTGGGC TACAGCACCCTGGACCAGGCACGGGTCAATGAAGCCTACGAGCATGCTTTCCACATGCTC AACCACAGTCAAGAGAACCAGAAGCTGGAAGTTGGCAACTCTGTTGCTGTGCACACGGGC TTCAACCCTTTGCCAGCTTTTCTGAATGATATCAAGCAGTTCTACACTGGTGAGGTCTTT AATGTGGACTTTGAGAAACCTGATGAGGCAAAAAACCAAATCAATCAACACATCGCCACT AAAACCCATGATAAAATCAAAGATCTGGTGAAGGACTTGGACCCACAAATGGCCATGGTT CTGATCAACTGGGTCTATTTTAGAGGACAGTGGGAAAAACCATTTAATGGCAACCTAACA ACCAAGAGGGACTTCCATGTGGATGAAAACACC
\end{abstract}

\begin{abstract}
ALD23I15
GCCGGGGCTTCTTTCCAGACTCAGACTAAGAAGCACACGAGCTTCATCATGGTGTACAAA GTAGCAGTGATCGGGGCGGGACCCTCAGGTCTGACCAGCATTAAAGCCTGTCTGGATGAG GGCTTGGAGCCGACCTGCTTTGAAAGCAGTGATGACATGGGTGGACTGTGGAAGTTCAAG GAAGTATCAGAGCCCAACAGGGCCAGCATCTACCGCTCCCTAACTATCAATATCTGGAAG GAGATGATGTGCTACAGTGACTTCCCCATCCCAGCTGATTACCCCAACTACATGCACCAC TCTAAAATCCTAAAATACTTCAGGATGTATGCAGATCACTTCAAGCTACTTTCTCACATA CGCTTTCAGACCTCAGTGAAGAGCGTCACCCAGAGACCAGACTTTACTCGCACCGGTCAG TGGGAGGTGGTCACAGAGGATAAAGATGGACTAGAGGAGAGGCATGTCTTCGATGCAGTA ATTTGCTGCTCCGGTCACTTCAACTATCCCAACCTGCCACTCAAAGACTTCCCAGGAATT GAGACATTTGAAGGAACATACTTCCACAGTTGGAACTACAAGGGACCAGAGGATATGTAT GGTAAGAGAGTGGTGGTCATCGGCATCGGTAACTCCGGAGGGGACATCGCTGTGGAGGGC AGCAGAGTTGCAGAGCAGGTATATCTGAGCACTCGCAGTGGTGCCTGGGTCATCCGTCAA GTTCCTGATGATGGCCGGCCAGTGGACAGG
\end{abstract}




\title{
Appendix D
}

\section{Table 4 sequences}

\begin{abstract}
ALD26D16
GGAGTTGCTGCGTAAAAAAGAAAGAAAGAAAGAAAAAGTCCGAAGCAACTCTAGAGGCAC ATCTGCAATTTAAGAAGAGAAGAAGGAAACATTTTTTCTCTAGAAGAAGACTATAAAAAG TGAAGAGTTAAAGGGGGAAATCATCCAGGAAAGAAGTAAGTTCTCTTTTTAAATAAAGTT GTATTTGTTGCGTTTTTGGGGGGGAGCTTTACGAGCCAATGAGACTTTAAGCAGCAGAGG TTTCAAATTTCAAACAAATGATGGAGTTTCATCTGAGCAGGAACTCTTAGCCTTTTTTTT TAAACAACAATGAAAACAGTGGATTTCTGATCTTACATCAGCCAAGAATGAACAACAAAG ATGCGCTCTTTGGATCTTTTAGTCTCCAAAAACAAGACGCGTAAAAAAACTAAACTTTGC GTAAAGTTGAATGAAGTGGAGTTAAAGTGAGAAAAGACAAACAAACGCACAAATTTTAGT GTTTTGTGGGGACAGATAAAAAAAACATGATGCTTTGTTTGGTGGGGTTCAACAGAAACT TTGCCCAGACATGAAACTTTACTGGAGCTTTACCTCAAGAAACGCGCATGGTGTGTGAGA GTGCGCGTGTGCATATATATGTGAGCATGTATGATCATATTTCAGCGCCTGTTTTTGATT TGTTCCTGCAGGAGAGCGGCAGTTA
\end{abstract}

\section{ALD38D17}

TTACGGAGGAATCTTTCGTGAGATCTTCTCGTCCCCGCTGAACCTCACGCTGCTCATGCC TCTGTCTGTTCCTGCTCTACCAAAGCTTCCTCGGGGACGGGCCACCCGACCTGGGCGAGC TGGAGAAGCCTCTGCCCACCATGAAAAAGAGGGATTTTACCCTGGTAGATCTGAAGCCCT ACGACGGACTGCAGGACCCGCGGATCCTCATGGCCGTCAACGGGAACGTGTTCGACGTGA CCCCAGGCAATAAGTTCTACGGGCCACAGGGACCGTACGGAGTGTTCA

\begin{abstract}
ALD24N21
GTTGGTACCCGGGAATTCGGCCATTACGGCCGGGACAGAGGAACATTTTCAGGTCACCCC CACCAACCAAACAATGTCAAAAAGATTTTTACATTTCAGAAGTTTATTGTCTGGTACCAG AAGTGACAGGACTGATGGTAATAATGGTAGGTTATTACTTACCTACCATTATTCTAGTTG CAAACAGTTACAAAATGATGAGGATGATCTGTTTAATAAGCTCCATAAGTAAAATTGGCA GCCTGTTTTGAAGTGAAAATGATTTTTGTTTTTGTGAAAATCCTCCTACTTAATGATGGT TAACTCGGTTAAGTCTTGTTAAAGCAGTAGTTGAATATGTTTGATAAAACCTGATTAAAA ATTAAGCTAAACCCTTCATCCATATTCACTTAGCACAAAAATTATAAATGAGGAAATTGT TTACCTTGTTTTAACTCTTTGTTAGCACTCCTGTTAAACAACAAACTGCTGATTTTAATA TTTATTTTTTTTGTAACAAACAATTATAGTGTCTTAGTAAACATCGAATGAGATTTACAG TTTGGAGTTTTACATGTAGACATCGTTTCCTGCATTTTAGTCTTTGACATAAGATAAGCT AGGCTAGATGATAAATTATTTTAGCTAGGAGTTTGGCTAGCTTAGTTCAACTAACAGCAG CTGTAAAGATACGAGAAACATGACAGCTTACTGATACAATTAAAGTGTCAACTATGACTA ACATACATATATATTA
\end{abstract}

\begin{abstract}
ALD28F11
GGTCTGTGTAAATAATATAACATGCAACAGCTAACATTACAGCAGGTAACTGTACCCCCA CAGCTGTGCCCTGTAACCACCCAGCCAATTAAAAAAAAAAAAAGAAAAAAAAAGGAAAGA AAGCCAATCCTGATATTTAATGAGCTAACACATTGCAGTGCCTTGTGAGCTTTTAGCAAG CTGCAGATTTGTAGAAAATGTGCCGTTAAAATAGTTAGTGTTTATTAAAGAATAAAGGTT
\end{abstract}


GACGTCTTGGGTAGCAAGATTAATAAAATCCTTATAATGGAATGTTAACCTATATTTTTT TCTGCTTATTTGCATTAACACAACATATGATAAAAACATGCCGTTAATCTTGTGGCTAAA CAACAAATTCCGCTTTATGGTTAAACAAATAATAAAAAAACACTTACGAATCTAAATGGA ACACACACAGAAATAGCCAAACAAGTACTTCTTTGTTTATTCACACAATGTCTTTCTGAA AACGTGATCTTGACACAATGATATAAAACACATATTAAAGTTGATTAAATATCCAGAGTG GTTTTTCTCCTCCCAGTCTTAAACTGCCATGTGCTTAAATGCCTCTACAGGCAAATAAAA TGGCTGGAGTGGGACAGGAATGTACATCCCTCCTTCTATTGTTAGTGTCTTTATGTCCAG ACGGATCTCA

\title{
ALD28L24
}

GTTGGTACCCGGGAATTCGGCCATTACGGCCGGGCGGGCTGAAGCACCCGCCCCAAAGAG CTGGGGGGGTTAGGGTGAAGGGAGTGGAGAGAGATTTGGAAAAAATGTT

\begin{abstract}
ALD35J11
GTTGGTACCCGGGAATTCGGCCATTACGGCCGGGGGACCACCAGAAGAAACAGCCGGGAC AACGAAATCCACAATGAAACTCTTCATGTTTTCTCTGCTCCTGCTTCCGTTTGTTGCTGT GGTGGAAACAGGCACAATAAGAGGTGAAAGAGAGAACGTGTTGAGTTTTCTACAGGAAGC TCTGAGCGAAGCTATTGAAAAGAGTGAACCTGCGGAGATCTCAGAACACAGAAACTCTTC AAGCAGACCCATTAAAGTCCAAGATTCAGAAGATGTGTCTGATTCAAAATCAACAGAGAG TGTGGAAAATGATAAATACCAAGACCTCGACAGTGATGAGATCCTGCTTCCTAAAGAGGA GCCGGTCCCGTCCGGGAGGAGGGCGGTCCGAGGGGGGCAAAGCATGCCGGTTCAAGGGGA TCTTCAAGCGAGTGACGATCAGAGCCATCGCAACAGCACGGGTGGAGTGATGCAGGTGTC TGAACGAACCAATCAGAGCCCGGCGGTAAGAGCCAACAGGATGCTGGACATGTATCCGAG CAGAGAGATGCCAGACTGGGACAGTTCGGAGGACCGAAGGTCACCTGCAGGGGACGAGAG CAAACTCACGGATGACGACGAGAGCAGAGACGACACGAGCTCCGAGACCGACCCGCTGAA GCCCAGCAGCCTTTCTGCTCCCGCCAGGAACCG
\end{abstract}

\section{ALD31H06}

TTTGTTTATGTGAAAAGATAATTACTCTTAATTCATACCTCTATTCCGATTCTGTTTGGA GTTTCTGGAAAGCCCCTGGATGTCCCGCAGGGTTGTGTCCCCTACGAACAAGATGTTGGC CTCAGGATGATCTTCTGCAC

\begin{abstract}
ALD30H07
GTGTTGGTACCCGGGAATTCGGCCATTACGGCCGGGGACCCGAGTTGTCGCAAAGCAGCT AACGTCAACGGACTGACCGGCTAGCTTGCTGAACAAGTTTCAAAGAAAATATAAATTAGT TTACCCATGTTTTTCATTAACTTCTTATCATTTTGTGTTATTTTATTAATATAGTTTCAG CTAACGCTTACTAATTAACAATATGGATGTTCTTCGCCCGCCTCTTATCAATATAAATGG GAAAATATATCGAAAAAACATCGTAAAGGAGGAACATTATGAGGAGGAAGATGATTATTC GTGCATGGGACCACTGGAGAGAGAGGATCTTGCTGAGGGTGAAACCTGTGATGGCCATTT AATTGAACAGACAGATAAGGGATATCGCTGCGCCATTGATGTCCCCAGTGTTCTTTACAA ATACATCATTGGGAAAAAGGGAGAAACACGTAGACGCCTGGAATCGGACACAAAAACATC AATCAGCATCCCAAAACAAGGAGTGGAGGGACAGATTGTTATCACCGGTTCACACAAAGC TGCAGTTTCTTCAGCGATCACTCGAGTCGAGGTCCTTGTGGAGAGTTTCCGAAGGAAACA GCCTTTCACCCACTTCCTGTCATTCCCGCTGAATGATCCCAAAGTTAAAGAAGGATTCCT GAGATTTAAAGATGAGGTGTTGCAGCAGTGTTCGCAGGACCATGGAGTGGAGGGAAGCAT CT
\end{abstract}


ALD27C17

AAAATTTCAAATTTCCACACACAG

\begin{abstract}
ALD38P21
GTTGGTACCCGGGAATTCGGCCATTACGGCCGGGGGCGG
\end{abstract}

\begin{abstract}
ALD01M11
GTTGGTACCCGGGGAATTCGGCCATTACGGCCGGGGCCAGTGGAGGCAACATAAACCTAA AAAATTGTGAATTTTATTACTATTTTTACACTTCAGAACTGAAGAAAAAAACATTCGTGC ACAAGAAAAATACTGGCTATAGTTTATACAGAATTTCATAAAAACCAGTACAACTGACAT TTTTTTCTAATTCTTATTATTATGTAGTCCTAAAGGTGTTTTTGGTAGAAAAGAAATTTG GGTAGAAAACAAGCTAGCATGAGCTAATGTTTTACTTTTTGTATTAGTATTTTTGTATTA GTATTTTTGTATTAGTAGTATTGATCACATATTTGCATTCAATTTATTAGTGTTGTGAGT ATAAAAACAAAAAAATCTGTTATGTTTGGTCACATTTTCAAAGAGCCACATGTGGCCCCA GAGCCGCAGGTTGCAGACCCCTGTCTTAACACATTACAAAACGGAACAAACACAAAGTTC AGGTGGTTTGACAGAGATGAAAGGTGTACGGAGAATAAAAAGGATGTGGTCTTCCACGGC ATTTGTGAATTCTGTAAATGTTACAGATTTGGATCTGGGACAAAATCCTTCAGACTTATT TAGTCTGTGTCAGTTTTGAAAAACATTCTTCTTGACATATTTTTTCAGACGGATTTTTC
\end{abstract}

\begin{abstract}
ALD08H16
TGTGTTGGTACCCGGGAATTCGGCCATTACGGCCGGGGGTTCAGAAGCACTGTGCCCCTG AAGAGAATTATTGTTGACGACGATGACAGTAAGGTGTGGTCTTTGTACGATGCTGGCCCA AAGAGCATCAGGTGTCCCATCGTCTTCTTGCCCCCTGTCAGTGGGACAGCTGAGGTGTTC TTCCAGCAGGTTCTGGCCCTGACAGGCTGGGGCTACAGAGTCATCTCGCTGCAGTATCCC ATCTACTGGGATCTCATGGAGTTCTGTGACGGTTTTCGTAAACTCCTGGATCACCTTCAG CTGGATAAGATCCACCTGTTTGGAGCATCTCTGGGTGGATTCTTGGCTCAGAAGTTTGCC GAGTACACACACAAATCTCCCAGAGTTCACTCTTTGGTCTTGTGTAATTCATTCAGCGAC ACGTCCATCTTCAACCAGACATGGACGGCTAACAGTTTCTGGATGATGCCAGCCTTTTTA CTCAAAAAGATTGTTCTGGGGAACTTTGCTAAAGGACCTGTGGACCCTAAAATGGCAGAT GCTATTGACTTCATGGTAGACAGGCTGGAGACTTTAAGCCAAAGTGAGCTAGCTTCACGA CTAACACTCAACTGTCAGAACTCGTACGTGGAGCCCCACAAAATAAAAGAAGTTGTTGTG ACCATCATATATGTGTTTGATCAGAGCGCTCTGTCTCTGGAGGCCAAAGAGGAAATGTAC AAACTGTATCCTAATGCCA
\end{abstract}

\begin{abstract}
ALD11C23
GTTGGTACCCGGGAATTCGGCCATTACGGCCGGGCACAGCAGCAAACTGTTTTTGTCAAA CTAAATGAATTCTCCTGGCTTAAGCTACATAAACCTTTTATATTTGCCCTGCACACACAG ATTTGTTTCTACACAGCTCTTGCTATGCCAATGGTCGATCCCAAGTTAGCTGTAAATATG AGATCTGGTATAAGGACAGGATGAAATAAATGGTTATAAAATATTCAAATGTTCATTTTG AGATGGCCCGTGACAATTTGCAAGCTGACACAGAAATGAGGCCCAAAATTGCAATTATGA GCTTGGAATTGTAAAGATTTTTTCTCAAACTTAAACCTATAAATGAAGTATAATGCATTA AAAAAGTTAAAAAAATGTTTTTACTACACTACTAATTTTAAAACCCGATTCAAGTGAAAG ACTAGTTGAGACTTTTCCTCAATCAGAACAACAGCAAACCAGACGTTTAAAGGAATAGTT CAGACATTTTGAGGTGGGGTTCTGTGGAAAGGTAATAAATTATTAACATTTAACTAGTGG
\end{abstract}


CACTTTTAAAGACTAAACTGATGAGCTAAAAGCTAGTATAAGCTAAGCTAAAACTAGTGA TGTGGATGTGAAGCAATGAACCANCACTGAACAATTTTATCAGTTGTTTTGGTTCATTTA TTCAGAGCTTTGTTTTAGTGACATCTAGT

\begin{abstract}
ALD20F24
GTTGGTACCCGGGAATTCGGCCATTACGGCCGGGACAGACAACTCGCTTTCAGAGAGTAC TGTCACTTCTTGGATTGGCACGTCCAGCTCAGCTGCAGAGATGAACACAAAATTTGTCTT GGCACTGGTCCTCGTTCTGCAGGTCTCTATGAGCCTGTGTGAGGTTCCTGCTCCATCCAA GGAGCTTGTTGACAAATACGAGACGATGAAAGCGACCTTCTACAAGAGACTGATAAACGC TTACGGCAAGCTGCAGGCCGCCATGGGCGAACAGCAGCAGGGGCAGAACGTCAGGGCTTA TGCCGAGTCTCTGCAAGGCAAGCCTGAGTTGCAAGCTGCCGTCAAAGTCGTCAGTGGTCT TGGATCAGAAGCAGGTCCTCTTGTGGACAAGGCCCGTACTTCACTGCTGGGTTTGTACGA ACACTACCTGCGCCCCCATGTCGGCGAAAGCCTGAGCAACGCCATCGACCAGATCAAGGT CGTCCTGGACCAAGTCATGCCTGCTGAGTAAAAATAAAATCAAATAAATAAAAATAAAAA AGACCAGCTACAGATAATGTCCAATGCCTATAATAGATACTAGCTCATAGAATCTGCATC AGTTTAGGTTGTGAGGAGCAATACTCAATGCAAATAAGATTCTCCACCTGTTCAATCAGA ATCTTGGCCAAAATCATGCAGAGTTATAAATAAAAAAAACAGCAAAAACCATCTATGCAA GTTCTGCATGTACAGTATTATTTGTTTGATACACTAACAAGTTCTGTGTGTTACTGTG
\end{abstract}

\title{
ALD09K12
}

CTTGTGTTGGTCCCGGGAATTCGGCCTTACGGCCGGGGGGGACCTTC

\begin{abstract}
ALD01B24
GTTGGTACCCGGGAATTCGGCCATTACGGCCGGGGCCGCTTCATCAGCCTGATCAGCTCT GGGAGGCAAAACAGGCCTGTCTGTCACTGTTTACTACCGTCGGTGTTTGACAAAACCAAC AAGATGACTTATCAGTTTCCTGCCCTGTCTCCGGAGAAGAAGAAGGAGCTGGCTGACATT GCTCAGAAGATAGTGGCCCCAGGAAAGGGGATCCTGGCTGCGGATGAATCGACAGGCACC ATGGCAAAGCGGCTGCAGAAGATCAACGTGGAGAACACAGAGGAAAACCGTCGCTTCTAC CGCAACATCCTTTTCTCCTCCGATGCCTCTATGGCCAACAGCATCGGTGGGGTCATCTTT TTCCACGAGACACTCTACCAGAAGTCAGACTGCGGGAAGCTCTTCCCCCAGGTCATCAAG GAGAAGGGGATCGTTGTTGGCATCAAGGTTGACAAAGGCACAGCTGCTCTCGCTGGAACA GATGGAGAGACCACCACACAAGGTCTTGACGGTCTCTCGGAGCGATGCGCCCAGTATAAG AAGGACGGCTGTGACTTTGCTAAGTGGAGATGTGTCCTGAAGATCTCCGACAGCTGCCCA TCGGGACTTGCTATAGCAGAGAACGCCAATGTCCTCGCCAGATATGCCAGCATATGCCAA CGGAATGGTCTGGTGCCCATTGTGGAGCCAGAGATCCTGCCTGACGGTGATCACGACCTG CAGCGGTGCCAGTAC
\end{abstract}

\begin{abstract}
ALD31K17
GTTGGTACCCGGGAATTCGGCCATTACGGCCGGGCAACTGTTTGATTATTTCCACACTCA TGCATTGCAATGCTTGTGATTAGCTGTGGCGGCCATCTTGAGTTGGGTTGGCCCCAAAAG TTAATCAGTTGTAGATGTACAACCAGTAAATCATTTCTGAGCGTTTTATTAAACTTTGTG TTCGGGGATTTATGCAAACATGCACACAAAACATTTTTTTCCTCTTTTCTTATTTTTATA CTTGATTCAGAAGAGTAAAAATGAGTCTCTGCCCCCCCCCCCCAAAAAAAAAAAAAAGAA AAGAAAACCCTCTCGCATTTAGTTCATGCAGGGACACAACATTTAGTTTAGGATTTATGG TGGCCATAAAGTCAGTTTTTTCCTCTAGAAGTGTGAGGTCAAATGGTGATTGGCTCAGGG CAGTGTGTGGTCAAACAGAGTGTGTGGTTAACATCAGTGGACGTAGAGTGAAATGATAAA
\end{abstract}


TGATGAGAAAAATAATCCCAACTTTAGAGTGTAAAACAAAAGGTTGAAAACCCCATAAAG CAACGGCACTCCTGAATTATTTTGTTGTGCTTAACTGTAGGTTTGTTGATGTTTGTTTTC CCCCTCCATATTGGATTTATTCCCCGAATCGACACAAGGTGGAGAATCAGATGTTGCAAT GTTGATTCGCGTCATTTTTACATTACGTTGTGCCATGTCAAAGATCATGGGAGCATGAGA GTCAGTGTGACCCTCCAGGC

\begin{abstract}
ALD27G15
TGGTACCCGGGAATTCGGCCATTACGGCCGGGACTGAGTCATTTCAGCACAAGTGATTCA AAACACTGAGTCATTTCAGCAAAAATAATTCAAAACACTGAGTCATTTCATCACAAGTGA TTCAAAACACTGAGTCATTTCATCACAAGTGATTCAAAACACTGAGTCATTTCATCACAA GTGATTCAAAACATTGAGTCATTTCATCACAAGTGATTCAAAAACACGGATTCATTTCAC CACAAGTGATTCAAAACACTGAGTCATTTCACCACAAGTGATTCAAAAACACTGATTCTT TTCACCACAAGTGATTAAAAACACTGAGTCTTTTCACCAAAAATGATTAAAAACATTGAG TCTTTTCACCACAAGTGATTCAAAAACACTGAGTCTTTTCACCACAAGTGATAAAAAACA CGGAGTCTTTTCACCACAAGTGATTAAAAACACTGATTTTTTTCACCACAAGTGATACAA AACACGGATTCATTTCACCACAAGTGATTAAAAACACTGATTCTTTTCACCACAAATGAT AAAAAACACTGATTCATTTCACCACAAGTTATTCAAAACACTGATTTTTTTCCCCACAAG TGATTCAAAA
\end{abstract}

\title{
ALD37G21
}

GTTGGTACCCGGGAATTCGGCCATTACGGCCGGGTTACGGCCGGGGACATAGTGGCAATA TGGTCTAAAAGAACACTGCAGTCTTTTTTGCAACTGAACATTGGTTGTGGGCGCGTGGCT TCATCCTGGGTTATCCCTGACTGCTCAGCAGGGAATCCTCTACCGTGACCCAAATCCTGC AGGTTAGTGCTGTTGTGGGTATAATCCTGAGTGGAAGCAGGATTTCTCCTTTCCTCTGGG GCTCTGATGAACGTATCTGTCTCCTGCTGATCCAGTTTCCTCTTCCTCAGCCTGCTCCTT ATTGGGGAAAACACACTGGCGGAAGTGAAAGGGGTAGTTTTCTTCTCAAACACACTTCTT CCACAGTTCTCAGCGGGCTGCATCTTCACTGGATCCCCTCTGGATGCGATGAAACCATTC GAGTTACTGGTCTGATCTCCGTTGACCACAGGGAGAAGTTGAGAAACAGCGGTTGACTCC AGGAAAGCTCTGCCCCTTCGTACGTCAGTTGTCAGAGAGGTTTCCTTCAGCGGTTTACGA GGATGACCGCTGGGTTTTTGAGGACTCGAAATGGGCGACAGAGTCAATGCCTCCGCGTCG TTGTGGCAAGGACCGAACTCCCGGTTCTCCTTGAATTCGGTTTTCTTCCCGGGCGAGTGA AATCCTGTTCCTCCGTGTTGCTCTATGATGTTATACAGCGAACCGTTGCATGTAAAAACT CCCCCA

\begin{abstract}
ALD28M08
GTTGGTACCCGGGGAATTCGGCCATTACGGCCGGGGCTTCGGCAGCTACATTGTGTGGAA AGCAGCAGCAGCAGCACGTTGTCGGCTCCTCCACGCTGAACTTTGCAGCGATTTGACGGC TCTCCTGTTCAAGGAGACTCAGCGCGACCGGCTACCCTTTCTGGCGTTTTCTCTGAGTTA CCCTCTACTCGCGGGAAGAAGAAAAATGTCTGCCTCGGCAGCGAAATCGAGTAAAAAGGG CTGAACTCGAACCATGACGGAGCGGACGAGACCTCCGAGAAAGAGCAGCAAGAAGCTGTT GAACACATCGATGAAGTTCAAAATGAAATTGACAGGTTGAACGAGCAGGCCAGTGAGGAG ATCCTGAAAGTAGAACAGAAATACAACAAACTCCGTCAGCCGTTCTTTCAGAAGAGGTCA GAACTGATCGCCAAAATCCCCAACTTCTGGGTCACCACATTTGTCAACCATCCACAGGTA TCTGCCCTACTGGGAGAGGAGGACGAAGAAGCACTTCACTACCTGAGCCGGGTGGAGGTG ACAGAGTTTGAAGACATCAAGTCGGGCTACAGAATAGATTTTTATTTTGATGAAAATCCA TACTTCGAAAACAAAGTACTTTCCAAAGAGTTCCATCTGAACGAGAGTGGAGACCCATCT TCAAAGTCAAC
\end{abstract}




\begin{abstract}
ALD35M22
GTTGGTACCCGGGAATTCGGCCATTACGGCCGGGGATTTGCGGAGGTAAGGCGGCTTGAA AGATGTCTTAATGCTCAGTGAACATTCAGTAAACTATTTTAATATTGGACAAAGCAGTGA TTTCTGTGCTTGACTGTCTTTTTTTTTTTTGGTATTTAGTCAAACTAATTAGTCATTATA TCAGCCCTGACCTGTGTCAAATGAAAACAACAACTTCTTCCGTGAACCAGGAGCTGACTG AGAGAGTGGACGGACAGGCAGATGAACTGAGGGTGAAGCTGCGAATGCTGGCACCCTGGG CTGGAGTTACAGATCAGAGTCTGACACTGCTGGCTTTGTGTCGCGCTTGAATGGGCACCT TAGGGAGAGACTGCGGTTCTGAATGGGAACGACTGGGGAAAGCCCTAGTGGTGGGAATAG AGTCTGGTAATTAAGGCAGTTATTGTGTGTCTGTAGGATGTGCTAGACAGCGAGGTCAGG AAGGCAGGCCTGTATTGTTCCACCAGCAGGGCCCTCACAGCATCACAGTGGTGCGTCAAA CTCAAGTATATTTGGAGAGAGCCTTAGATCTTCCAGAGAAGAAGAGGAATTTCAACAATA AACCGCACAGGCAGCCCTATATCGTCTCCACGTCTGTG
\end{abstract}




\title{
Appendix E
}

\section{Table 5 sequences}

\begin{abstract}
ALD41H13
TGTTGGTACCCGGGAATTCGGCCATTACGGCCGGGGAGGAGTGCATGCGGACTGTCGGCT TCTATATCAGAAGGAAATGAGGGTCGAAGAACTTGTTTTTAGCAATAGAGTGGGTTGGAA GTGGATATTTGATGTTAATAATTGTTTTTTTTTTTTGAACATCTTACAGGCTCTGACATG TTGAATAAATTCGAAACTAAGTTTTTGAAAGATTCCATAAAACATGAAACAAAAAAATCT GTGAAATTGCTCTATAAATCCATATACACAAGAATTTGTTTGAAAATGCAAAAAAAGATG AGAAGTTCGGTGTTTATTGACCAAAGCTATAAAGATTTAACTATGGAGTACCCTCAACAC GACAGTCACTACAACTACATTGATTAGGATGAACAAAAACACCGGCTCTTGACCGCCTAC CACACAATGCACCAGACTACCACACCACCACACCTTGCAACAATTGCCGCAAAAATATAA CAAAGCCACTACATAATGCATTAAAGACCCCCATTACATTTAGTGCCAATCACTACACAA CGTGGTCCCAGCGCGAGTTTATTACATTTTGAAGCCTGCCACATTATTACACAATATGTT GTCACCACAAAATGAGGTCCCACACGCCTTCTTGCTGTGAGGCGTCGGTGCTAACCACCA A
\end{abstract}

\begin{abstract}
ALD21G08
GTTGGTACCCGGGAATTCGGCCATTAGGGCCGGGGCGTGGGGGGGGCGGAGCTCTGAGGC CACAGCTGAGCTTTCAGCTCGTCACTGCTGGGACAGCAGGGCGTTCCTGTTGGCCTTCAT CTTCTCCAGACGCTTCACCAGGTGCTGGTTGGTCATCTCCATCTCCTCCAGCTTGTCCAG AGCCGTGCGCAGCTCTCTCTGAAGCTTCCGTTTCTCCGCTTTCAGCTCGTCTTCTACTTT CTCTGCGTTGTCGGCTGCTGCTTTGTAGCGCGCCGCCTGGCCTTCAAGCCTCGTGATGTT TTGTTCCATCGTACCCATTTCCTGCTCCGCTTTAGACAGTTTGAACTTGTATTCACTGAT CTGCCTGTTAGCGTCTCTCTGCAGCTCGATGAGGTGCAGGTCCGTCCCGTTCTCCAGCTT GTCGCCGTCGGCGAGCGCTCCGTCCAGCTTGGCGTGTTTCTGCCGCTCCTCGTCCAGCTG GTTCTTCAGCTTCCTGATCTGAGCCAGCAGCTCGTCCTTCTCCTCGGCCAGCTTCAGTAA CCTGACGTCCAGCGAACCCTCTCCCGCTGACTCCAGAACCTGCGCGGCCTCCTGGGACAC CAGGGTGATCCCCGGGGACAGAGGGTTGTGGCTGACGTCTCCGTTG
\end{abstract}

\begin{abstract}
ALD39G18
GTGTTGGTACCCGGGAATTCGGCCATTACGGCCGGGGGGAACCAGTGGTCTAAGCAAAAC ACTAATGGTAACGAGACTTCGGTTTCATTGGCAATTGTGAGCAGATACTCAAGCTCAACC AGACATTTTCTTTTCAACTGCTAAAATTTGACCAGTGTGAACCTTGCTTAAGGCATTCTG ACTGTATTTAGCCAAATACACAAACAGCAACAGGCGTCATTTGGCTAAAACGTTGAACTT CCTGTAAATGTTTCTTGTTATTCTCAGATAAAATGATGGTCATTGGAAGTTGATTTTTGG ATGTCAGCTAGCACACGAGCTACTGTTAGCCTCATGGTTTGTATTTGGCTAATTTGGAAA TACTAAATATTGTTTAGGACAGACCTAGTGCAAAATGTTTTCTGCACTAAAGTAGCTTTT TCTCTAATTTGGTCGTAAACAATTAAGTCTTCTAGCCTACCTTGACTTTGCTGATTCTAG TACCGTAGTCTAAAATGATGTACCATCCAAAAAAAGTGATTCCATCAAAATTGTTATAAA TACTCATGAGCTGTCTCAAACGTCTCACACTTTGTAGGAATCTGACAGAGTCACCCTGTC CAATAATGTATGAAGTCCCAGATTTAGATCTGCTTATTAACATAATGGGCATTGTGTTAC GTCTCTTCTATAGGTCACACTCA
\end{abstract}

\section{ALD41B02}


GTTGGTACCCGGGAATTCGGCCATTACGGCCGGAGTATCGTTTGTTGATCGATTTAATGT ATAATTTATTTGGACCATTTTTTTCCCCCTCTTCCACAAGGTGTTTGTTATAAGACACAC TCTTTCAAAAAGTTTTCTTTATAAATAATTGGATTTGTTTTTTTCCCCTCAAAGTTAAAC ATTCCACAAAGCGACAACATAAAAATAAATTCTGTAAATCTTTAGCGGTATTAAGTTTAC TTTTTTTTTTTTGGAAAACTCCATGTCACTGTGCCACACTGATTTGTTTTATTTTTCCCT TTAGTGTTTGTTTTTATGCAAAGCTCTCTTTATTAAGCTTTTACTTTCTGTATGGAGAAT TTTCAGACTGGTGCTGTTCATTTCAGGAGAAACACCAGATTTATTTTTCCTTTTTTTATT TTATTTGATTGTTTTTAAAATTCAGCAAATGTTGTGTATGAGGGTGAGAGAAGTAAGTGA GCCGGAGCGGTCGTGCCCGGCAAAGTTGATAATTTTTATTTTATTTTTAGGTTTTCTTTT TCAATGTGTAAGAGTTGGTGATTATTATTATTATTTTGTTATGATTAAGTTAAGCTCATT CTGTAACCAACAGGTGACGAGCTCTCAGTGCTGTAGCCATTTCACCAAAGAGGGTTGTTG ATCTATCACAGCGCTGAAAAAAGTAAAACTTGTGCTCG

\begin{abstract}
ALD14E08
GTGTTGGTACCCGGGAATTCGGCCATTACGGCCGGGGCCACAGGTGGAGCATCTGCCTCT GCTGGACATCAAGGTGTCAGACTTTGGGGAGAGCAACCAGCAGTTTGGGTTTGAAGTGGG ACCTGTGTGTTTCCAGGGCTAAACACACACACACACACACACACACCATCCAGAGAACAC ACACACCTAGAGACACACATGATAGACATACAGGCTCAGTAATTTGTAAATTAACTTGTA AATGATGACACACACGCATACCTACACTGTGGGAAAGCAACATCCTCCCAACAACTGCTG GACCTAAAGGTCAAGAGGAGAAAAATGTAAAAACTTGTGAGGAGAACATCGACTGGGAGC AACAAGGAGGAAACAGAAGAACTTTTTTTTATTTTAGAATAAAATGATGATTTCAGGAGC AGGGACCCAGGCCCGGGTTTAAACCTGAATACCCTCATCTCCAACCCCCCCATCTCCTCC GCTGACACCTTTCTCCACTCCTGCTCCCCCCCGTCTTAAAATAAGAGTCACTCTGAACCT GTGAGCAACATGAAGAACACTCCGGTTATAAATCTGTTCAGTTATTTATTTTGGCTCTTT GATCAGATCTGAAACTAATTTTTCATTCACCAAATTTAAATTAAATCTTACATGTCCACC GACCTGTCAATCATGTTGCTTACATCACTTCATGTCACAACAAA
\end{abstract}

\begin{abstract}
ALD41D24
GTTGGTACCCGGGAATTCGGCCATTACGGCCGGGGATGTTTGGGTGGGGGGACTTCCACT CCAACATCAAGACCGTCAAACTCAACCTCCTGATCACAGGCAAGATCGTGGACCACGGGA ACGGCACGTTCAGCGTCTACTTTCGCCACAACTCCACAGGGATGGGGAATGTGTCTGTGA GCCTGGTGCCGCCTTCCAAGGTTGTGGAGTTTGAGATCGCTCAGCAGTCCACGCTGGAGA CCAAAGACACCAAATCGTTCAATTGTCGCATTGAATACGAGAAGACGGACCGCAACAAGA AAACCGCCCTGTGCAACTTTGACCCGTCCAAGGTGTGCTATCAGGAGCACACGCAGAGCC ATGTGTCGTGGCTGTGCTCAAAACCCTTCAAAGTCATATGCATCTATATCGCCTTTTATA GTGTGGACTACAAACTGGTGCAGAAGGTATGCCCTGACTACAACTACCACAGTGACACAC CGTACTCTTCCACAGGATGACCTAACTGTTGTATATGCATCAGTCTTTTGTTTTACCTTT AAACTATGTCAATGTCCAACTCTAAACTCATTGTTCTGAACTACCTAAACAACTTGATAT GTTGAAGAGTCAGCTC
\end{abstract}

\title{
ALD20J24
}

GATTCGGCCATTACGGCCGGGGAAGCTGTTTTTCAGTTCTTTTCTCAAAAGTGGGCTTCT TTCGCTCTACAGAAGACAGAATGAGTATTCATGTGGAAAAAATGGATTTTTTCCAAAACT CTTTCCCTACTAACAAAAAAATGTTAAAGAAATGAATGTTGACACTTTTTAAGTTAATTC TCATATCAGAAGCTGTTTTTCAGTTTTTTCTCAAAAGTGGGCTTCTTTGGCTCTATAGAA GACAGAATGAGTTTTCATGTCTAAAAAATGTATTTTTTCAAAGTCTTTCCCTACTAACAA AAAAATGTTAAAGAAATGAATATTGACACTTTTCAAGTTAATTCTGATATCATAAGCTGT 
TTTTCAGTTTTTTCTCAAAAGTGGACTTCTTTCGCTCTACAGAAGACAGAATGAGTATTC ATGTGTAAAAAATGGTTTTTTTTCCAAACTCTTTCCCTACTAACAAAAAA

\begin{abstract}
ALD40E16
GGAGGGAAAGAGTTTTGGAAAAAAATCCATTTTTTAGACATGAAAACTCATTCTGTCTTC TGAAGAGCGAAAGAAGTCCACTTTTGAGAAAATAACTGAAAAACAGGTTCTGATATGAGA ATTAACTTGAAAAGTGTCAACATTCATTTCTTTAACATTTTATTGTTAGTAGGGAAAGAG TTTTGGAAAAAATCCATTTTTTTCACATGAAATCTCATTCTGTCTTCTGTAGAGCAAAAG AAGCCCACTTTTGAGAAAAGAACTGAAAAACAGCTTCTGATATCAAAATTAACTTGAAAA GTGTAAACATTCATTTCTTTAACATTTTATTGTTAGTAGGGAAAGAGTTTTGGAAAAAAT CCATTTTTTTCACATGAAATCTCATTCTGTCTTCTGTAGAGCGAAAGAAGCCCACTTTTG AGAACAGAACTGAAAAACAGCTTCTGATATCAAAATTAACTTGAAAAGTGTCAATATTCA TTTCTTTGACATTTTTTTGTTAGTAGGGAAAGAGTTTTGGAAAAAATCCATTTTTTCCAC ATGAAAACTCATTCTGTCTTCTGT
\end{abstract}

\begin{abstract}
ALD41D11
GTTGGTACCCGGGAATTCGGCCATTACGGCCGGGGACTTTTGGATTTTTAAGATTCCAAC ATGTGCAAAGGACTCGCAACACTCCCTGCAACGTGCTTGAAAAGTGCAAAGGACATCAAG CACAAAATAAGCTTCTTACTGCAGAAGCCTGAACCCCACGCAGCGGATCAGAGAGAGATA AAGGAGAAAAGTGCCACAGCTGCTGCAAAGAGACTGCCTGATCCAGCCGATTTGGAGAAA TGGAAAGA GTCTTTCAGCCACGTGATGAGCAACGAGGTGGGTCGAACGGTCTTCACCACC TTCCTGAAGTCAGAGTTCAGTCAGGAGAACGTGGACTTCTGGATCGCTTGTGAGGATTAC AAGAACACTCCACCATCCAAGGCGGCGACCAAAGCCAAGCAGATCTACCAACAGTTTGTT GAGGCAGATGCTCCCAACGAGGTAAACTTGGATGCAGTGACCAGAGAGAAAACGAGGCAG GAAGTGGAGGACGGCAGCCCGTCCTGTTTCGACGAGGCTCAGAAGATGATTTACAAGTTG ATGGAGAAAGACTCGTACAGGCGCTTCCTGAAATCCAAACTGATCCAGGATCTGTGTCGG AAAGAGTCCGGCTGCCGCTGGGCTGACAGCAGGCCGATGTTAGCTGGGGGGGCCTAAAAC TGGAAGCAGGACCCGGAGACTCAGAGAGGAGCCAAGGATTGATTTACTAAATTAGGACAA TAAGACAAAAGTTA
\end{abstract}

\begin{abstract}
ALD38E15
TTGTGTTGGTACCCGGGAATTCGGCCATTACGGCCGGGGGCAGGAGGAAGGCAACATGGC CGAGGCGGAGGCAAGCGAAGTAAGTTACGGAGGAATCTTTCAGGAGATCTTCACGTCCCC GCTGAACCTCACGCTGCTCAGCCTCTGTCTGTTCCTGCTCTACAAAATCTTCCGCGGGGA CAGGCCGCCGGACCTGGGCGAGGTGGAGAAGCCTCTGCCCAAGATGAAAAAGAGGGATTT TACCCTGGTAGATCTGAAGCCCTACGACGGACTGCAGGACCCGCGGATCCTCATGGCCGT CAACGGGAAAGTGTTCGACGTGACCCGAGGCAAGAAGTTCTACGGGCCAGAGGGACCGTA CGGAGTGTTCGCAGGCAGAGACGCATCTCGAGGCCTGGCGACGTTCTGCCTGGAGAAAGA CGCCCTGAAAGACGAATACGACGACCTGTCGGACCTTAACGCCATGCAGCAGGAGAGCCT GACCGAGTGGGAGACTCAGTTCACCATGAAGTACGACTACATCGGCAAGCTGCTGAAGCC CGGAGAGGATCCGACAGAATACACCGACGACGACGAGGGGAAAGACAAGAAGACGGACTG AAGCAGCTACAAAGTGACGAGGAGGAAGAACGTTAAAGATGCCTTAATGTTTGGACTTTC AGAACTTCTGCCTCCTTTTTTTTAGGCTCGTTCCCAGAAAACCTTTTGCTTTTTACATT
\end{abstract}




\begin{abstract}
ALD39G15
TGTGTTGGTACCCGGGAATTCGGCCATTATGGCCGGGGGAGGGGTTTTGGAGGAAATGGT TTCCGTAGCAGTGGGCACCGAGGTGGTGGAGGTAGAGGTGGATATGGAAACAACAGAGGA GGACAGAAACGGACTTTCAGCCAAGCTTTTGATTACTGAAGCATCAGCATCACCTGCCTT TGACCTGCAGTCTTTGGAACGACTCGTCCCTGCTGTTCCACAGAACAAATCTGAATAAAA CATTTTAATGCCAAACCCTGAATATCTTCACGTCATAAAGTTTTGTAAACCTGCCTGTTA CTAAAGCTAACATGTGACAACACGGTCAACAAATTAGGATTGGAATGTCGCCCTTGGAAT TTTTTAGTATTTACCTTTTTTAAACTGGGTGTTTGGGGAACAGCAGCAGTAATCAGACTC GAGCGTTTGTTAATAAAGACAGATAACCTAATTAACAGGTAAAACTGACATTTATTACCT GATTCTAGTTTGAGTTTGTTAGATCAACTCTGTGTGTCTCCATCATATTAGGTGTTCTGT ACTTGTTTTGCACTAAAAGAGCAATGAAATAGAAGCACTTGGAGCGCTGTGAAGAATCCT TCAAAAAATTCCAGATCGTCACCAAAATTTAATGGTGTGTTCCTTTGGCCAATACCCACC TGTGGTAAAAAATTTATGAAAATCCATCGAT
\end{abstract}

\begin{abstract}
ALD21F15
TTGTGTTGGTACCCGGGAATTCGGCCATTACGGCCGGGGGATGAACAACTTGGAAAAGTT TTGTGAAGCGGCTTTCTGTGTGAACAGGAAGAAACGGTCAGCAGGCTGAGGTGTAGCAGG AACACAGGAACCTTTGGAGTCAACAGGAGCTAAGATCTGGACCTAGAACTGAACCATCGC TGGGCTTCTTTAAGGAGTGTTTCTCATTCTGGGGGACCCGCCAAGGCCCGGCCCGGACCT GTGAGCCTCTGGACCTAAGCAGACCAGAGCCATGGCCTCTGGTAAGAACAAGAACCAGAC CTCCCTGGTCCTCCATAAGGTGATCATGGTGGGCAGCGGCGGTGTGGGGAAGTCTGCACT GACCCTGCAGTTCATGTACGACGAGTTTGTGGAGGACTACGAACCCACGAAGGCTGACAG CTACAGGAAGAAGGTGGTTCTGGACGGCGAGGATGTCCAGATCGACATCTTGGACACGGC GGGTCAGGAGGACTATGCCGCCATCAGAGACAACTACTTCCGCAGCGGCGAGGGCTTCCT GCTGGTCTTCTCCATCACGGAGCACGAGTCCTTCACGGCCACGGCCGAGTTCAGGGAGCA GATCCTGCGGGTGAAGGAGGAGGAAGGCATCCCTCTGCTCCTGGTGGGGAACAAGTCAGA CCTGGAGGACCGCAGGAAGGTTTCTGGGGACGAGGCAGCAGTCAAGGCCGGCGAGTGGGG CGTTCAGTACGTGGAGACATCGGCCAAGACC
\end{abstract}

\title{
ALD28B18
}

TGTGTTGGTACCCGGGGATTCGGCCATTACGGCCGGGGGGGGGGGG

\begin{abstract}
ALD32G09
TGTGTTGGTACCCGGGAATTCGGCCATTACGGCCGGGGCCTTCTTTCTGCTTCACGTCAA GGACTTTCCTCATGTGAGGGACCAAAAACCAGCCAAAGTTTTACATGATGAGTTTCTGTC AAAGATAGAAAAAAAAGAAGTTTTCAGCTGTGGTGTGTCTTTAAAGCCTGACAATCTGAT GATCAAACAGGATGAAGAGTTGTCTCATCATCAGGAGCTGACTCAGGATGATCGAGAGAT TTCAGCCTCTTCCTCCTGAACCCGCCGTCCCAGATGATGAAGATGCCACAAAGGGAGCTG ATCGACGGGATCTGAGCCATGAAGAGGAGCCACCGGACGTTCAGTGCTGTGACTGAAGCT CCTCACGAGGATGTTCTCGACAAACCGGAGGAGTTTAAAGCACAGATCAGGGAGAGCCGT GACTTCTGTCTACAGATCCGAGCAGCTTTTAAAGCCCAGCTGAACAGACGATGATGCTCA CAGGTTAAAACAATGAAGCAGCTGTGAGAGGGAGGAGCTGAGGAACGACGTGGAGGAAGC AGGAGAGGTCCTGTCTGCTGAGAGCATCGTCAGAGACCACCACCTCATACACAGAGACCA
\end{abstract}


CCGCCTCAGACACACAACAGAAGCACAACCTCAGTGTTGCAGATCAAGAAGAAAAGATCC AGATCCTGCATAGAGGTGCGAGCTGCATCATCCATGAGCTGGAGGAAGATTAAGACAACT GTAA

\begin{abstract}
ALD14E16
GTTGGTACCCGGGAATTCGGCCATTACGGCCGGGGGAGAATTAACTTAAAAAGTGTCAAC ATTCATTTCTTCAACATTTTTTTGTTAGTAGGGAAAGACTGAAAAAAATCCATTTTTTTG ACATGAAAACTTGTTCTGTCTTCTGTAGAGCGAAAGAAGCCCACTTTTGAGAAAAGAACT GAAAAACAGTTTCTGATATCAGAAACAACTTGAAAAGTGTCAATATTCATTTCTTTAACA TTTTTTGTTAGTAGGTAAAGACTTTGAAAAAATCCATTTTTTAGACATGAAAACTCATTC TGTCTTCTGTAGAGCATAAGAAGCCCACTTTTGAGAAAAGAACTGAAAAACAGCTTCTGA TATGAGAATTAACTTAAAAAGTGTCAACATTCATTTCTTCAACATTTTTTTGTTAGAAGG GAAAGACTTTGAAAAAATCCATTTTTTAGACATGAAAACTCATTCTGTCTTCTGTAGAGC GAAAGAAGCCCACTTTTGAGAAAAGAACTGAAAAACAGTTTCTGATATCAGAAACAACTT GAAAAGTGTCAATATTCATTTCTTTAACATTTTTTGGTAGTAGGGAAAGACTTTGAAAAA ATCCTTTTTTTAGACATGAAAACTCATTCCGTCTTCTGTAGAGCGAA
\end{abstract}

\begin{abstract}
ALD28B21
TGGTACCCGGGGAATTCGGCCATTACGGCCGGGGGTGTTAAAACGTGTTGCTCCGCCTCC AAAACACATCTACAGAAAAAAAGTTCCTGCATTTTAATTATATTTCTTTTAAAAAATAGT TTTTTAAACTTGGGAAAACTTATTTTTTATGCTTTATTTTGTTTAAGTAAGTTTGTCCAG TAAACTTGTTCGAAGCGGTCAGGGGGTTGTTGTTGTTTACTGGCATATTTACAGCACGTG GAGTCAAATCTCGGGTAAAAATTCATTTTTACCGAAGAAACGAAAGACAGCAAAACTACG GAGGAGTTTGTGACTAACAAAAAGCATGGCAAACCTTCCGGACAAGAACTCCAGCTACAA AGACGTGACCTACTGGGATGAGAGATATTCCAGGGAACAGCAGTACGACTGGCTGGGAGA TTTCTCCAAATTCCAGCATCTTCTGGAAAAAGTGATAAAGAAAGAAGATTCAGTTCTCAT CCTGGGTTGTGGAAACAGCAGCATGAGTGGGGACATGTACAGAGCCGGGTACCGCTCCAT CACCAACATAGATTACTCCCCCGTGTGCATCAGCACCATGAGTGCCAGATACAGCCACTG TGCCGAGATGACCTGGCATCAGATGGACGTACGCCAGCTCTCGTTTGACGACGCATCCTT CGACGTGAACCTCGAGAAGGCAACGCTCGATGCCATCATG
\end{abstract}

\begin{abstract}
ALD28A20
GTTGGTACCCGGGAATTCGGCCATTACGGCCGGGGGAAAACAGCATAGAGACTATACAAG CAGACACATTCCGACATCTACATCATCTGGAAGTATTGCGGCTGGGCAGGAATGCTATCA GGCAGATTGAAGTAGGGGCATTTAATGGCTTGACTAGTCTCAATACTTTGGAGCTATTTT ACAACCGATTAACAGTCATACCCAGTGGAGCTTTTGAGTATCTCTCAAAGTTAAGAGAAC TGTGGCTTAGAAACAATCCTATTGAGAGCATTCCCTCTTATGCCTTTAACCGTGTCCCTT CACTCATGAGACTGGACCTGGGTGAACTGAGGAAGCTGGAGTACATCTCTGATGGAGCAT TCGAGGGTCTACATAACCTCAAGTATCTAAATTTGGGGATGTGCAGTCTAAGAGAGTTCC CTAATTTGTCACCTTTAGTTGGATTAGAGGAGTTGGGGATATCAGAGAATGTTTTTCCAG AACTGAAGCCTGGGGCCTTCTACGGGCTAAAGAATTTACGCAAACTTTGGATTATGAACT CTGCCATCACAAACATTGAAAGAAATGCATTTGATGACATTACAGCTTTGGTGGAGCTCA ATCTAGCCCATAATAATTTATCATCCCTGCCCCATAACCTCTTCACACCTCTACAGTACC TGGTGGAGCTGCATCTACACCACAACCCTTGGCGCTGTGACTGTGATGTAGTTTGGCTCT CTT
\end{abstract}

\title{
ALD09H02
}


CTGACCCGCAACTGTAACGGATCAACGTGTATTACACCGAGGCCTCTGGAGGGACAACGG TTCCTCGAGCAGTTTGGGTGGACCTGTAGCCAGGCACTATCGACTCTGTGAGGACTGGTC CTTTCTGGCAAGTGCTCTCACGTGACAGCTTTGTCTTTGCC

\begin{abstract}
ALD26C03
GTTGGTACCCGGGGAATTCGGCCATTACGGCCGGGAAACGAGCCGTGGAGCAAACCCAGG TCAACATCCAGTGGGTCAGCGAGAACAAAGACGTGGTCCTGCAGTGGTTCCTGACAGAAA CTGCCGACATGGAGTAACAGATCTGTGCTTCTGACAATCTGGGTCATCAAATGACTGAAC AGTAAACCTGATTTCACCAGAACTTTCAGGTTTCTGTGAGGTTCAGCTGGCGGCCAGCCT GAACCACCAAGTATTGACTGTTTATGTAAATACTAATGTGCATTATTTATAGACTGTAAT CACTAAAAACAAACTTCACTTTGTTTAATAATGTTAAATGTGCTGCTCATAATTGTAAGT TGTCTCTTTAAGAAAACAGTTTTATTTCTCTTGTTGTTGCTGCCTGTGCATCAAATCGTT CCACAAACTGAACGTTTTCATGGTTTAAACATCCGTTTTCTTCCCTTCTCATAAGATGAA ATCAGTTTAAAAATCAGTTTCAAATCCCGCTGCTAATCTTTAAACATCTGTAAAAAAAAA TCTCTGCATGTTTCCACAAACAACTTCATGGTAAAGTTTTGTTATTTAACATAATTTTCA TGAACTTAATGTTAAATCTAATCAAAGATCTCAATCATTGCTGCTGCTGCTGTACTGGAA ATGTTTGAAAGAAAACATTGTTAAACATCTCTTTTCTTTCTTTTCCTGAAA
\end{abstract}

\begin{abstract}
ALD29M01
GTTGGTACCCGGGAATTCGGCCATTACGGCCGGGCAGCCTGGTGTAGCAACTCTCTGCCC CTCTGTAATAAATGTCTTCACACAGCGCACACAAGGAGCTCTGTTCAGCTGCATTCAGGA CTGTTCTTTCTACATTACCCAAGTTTATCATATTCTTCCTCTCTGCGGAGTTCTGATCTC ATTCAAGGGGCATAACCTGTTAGGGTCTGGTGCACTCATAGGGATTGTAAAATCAAGGTG CACAGATGATCAGACTTGTATGTTAAAAGCAAACTGGAAGGATTTTGATTTTGTTGTTGA TTCTGTTAAAAATGTTGTATATTTACTGGTTCAGCAGAGCATCAGTAGGCAGTAAAACTT CTGATTTACAGTGTAAAGATCTTTATATAGACATTTGAAATAGACATTTGAAATAAGGCA TTATTGGTGACTACAGACAATATTTTCAAACCGATGCCAAATCGCTCAACCTTTGGTGTG ATTTTAATCAAACTGAAATTAGTGCACTTTAAAAAACGTGATGCTTTAATTAATATTTCT TAAATTAGAACAAAACCAACCACCTCAGCCAGTTATGAAATTATTAACCATTTGTTGTAT CTGTGCTGTAAAAACAGAAAACATGCACAAATCGTATTATTCAGGAATTAGTAAAATAAA TTGAGTTTGTTTAAT
\end{abstract}

\begin{abstract}
ALD36D24
GTTGGTACCCGGGAATTCGGCCATTACGGCCGGGGATTTAATTTTTTTATTTGTAAGTTT GGACATTCACATTTTAACTTCAATCCAAGTTCCTTCAAAGCGCAGCTGGTTGTCACCACG CTCGGCTTTGTTCTGGCCGCAGCTCACCAAACAGTCGCCAGTTCGGATTAAACTTCGGGT CTGAATGTGGGCCAGTTTGTGTTGATGACTGGCAAATGTAGCAACAGTTGGATTGTGTTG TGGCTCGAGTTGGACTGAGAGATTGACCTGGTTTGTTATTGGCTTTTTTTAAGGATTTTC TGTGACTTTAAAACAGGAAACAGTATCAGCTGCTGGGGGTTAGCAGCTGCAGAAGTAGCA TAAGTGTGGATGGGGAAGTGAAATTGCTGAGTTTAACTTCACTTAAACCAGTTCCTTCTA TGTTTTAGGTAAAATCGATAAGCTGAAGAGTTTTTTATGGATTGATTCCACTCTAGTCTT GGTGATGCTAGACCAAAACGAGCAAATTATAGCAAATGAGTTTGGATGAGATCGTTCTGA GCCATGAGGACGATAAATTGTGTGGATTGAATAAAAGGACACAAGTGGAGTTCAAGGACA AACATAAAAAAGGGGAGGTTTGCATGAAGCAATACGCATGAGTGAAACGCCAAAGTGTAG CTCTGCACAAACCGCTCGAGCTCTTCGTTTTCC
\end{abstract}

\title{
ALD33L21
}


GTGTTGGGTACCCGGGAATTCGGCCATTACGGCCGGGTTCTTCTTTAATGAAACGTTTCG TCTCTCTTCCAAGAGACTTCTTCAGTCTGAGAGTTTCAGGCAAAATTCAGGCTTTTATGT CCTGCCTCCAGGTGACTGATACTCACATCACATGACCATCGTTCACAGGTGACTCCCACT CACATCACCTGGCTATGACTCACAGGTGACTGATACCCACATCACATGGCCATCGTTCAC CCTAATTAATCCCTTTTGTTAACAGGGGGTGGAGTGATACTGCCAATGGCAGCGCTGTAG CAGGGGGACAGCTGAAAACGGAGGCCCCCACCCTGTTTAATGACGGACGGTCTTTCTTTA CAAAAATGGCTTCTTTTACTCCTCTCTCAAACCAACGTTCCTCCCTATCAAGAATGAGAA CATCCTCATCTCTGAAAGAGTGACCGCTGGCCTGTAAATGAGTGAAAACTGCAGAGTTCT GGCCATTAGATGTAGCTCGTCTGTGTTGGGACATTCTTTTGAACAGTGGCTGCTTTGTTT CCCCGATGTATAACTGGTCGCAGTCCTCCTGGCACTTCACAGCATATACAACATTGCTCT GTTTGTGCCGGGGCACCGGATCTTTGGGGTGGACC

\begin{abstract}
ALD03D04
GTTGGTACCCGGGATTCGGCCATTACGGCCGGGGACCTTCCTGGGCAAAGTCTGCATCCT CTCTCTGTGCATGCATGGGTTTTCCCTAGATGTAGGTGAGAGAGTGAATGCTCGTTTGTC TGGTTGTCTCTACGTGGCCCTGTGATGGACTTGTTGACCTGTCCAGGGTGTACACCGCCC CCCGCCCTCCTTCACACAGTGACTTATGGGCAAGAGTGACCCCGGCCAACCCGAAAGGAA GAAGCAGGTAAGGATAATGGATGGATGGATACTTGGGCCTCTGAATCTTCATATGACAGT GTAATCTCTTTGATCTGTTCATTTCATAGGTTACTACAGTTATTTTAAGCTGCTCTTGTT TGTGCCTTGCCTTTCCACTTCTTTCCTTGGGTGGTCTGAAACAAGGATGTGGTGAAAAAT GATAACATTTCCAGTCTTCCGATTCACAGGAAGGTCCACATCTCAGTCATTTCAAAAGCA CATTAAAAGTTAGTATCTACTGGACCATTTACACTTTCAATTCAACCCTACCTTGGCCCA GTGCGGGTTCAGCATCAGTCACTTTAGCATTGTAAAGGCCGACTCAACTGGTGGGGCCCA ATTGACCTGGACTTATTCTACCCTGGCCCATGTTGTTTGGATTGAGCAGAGAGCCACCTT GATGTGGGTG
\end{abstract}

\begin{abstract}
ALD33D21
AGGCGCGCCATTGTGTTGGTACCCGGGAATTCGGCCATTACGGCCGGGGGCCCATTAGAA TCAACATGGCGTCCAACTCGGTTTTATTCGTTTGTTTGGGTAACATCTGCAGGTCACCCA TTGCTGAAGCTGTCTTTAGGAAGATGGCAGCAGATGCTGGCGTTGTCGATAAGGTAATAT CATTTTTAGTTCACTGCCGCTTATGGTAATATTTATTTTTACCTCAGTGAAATTGTTTAT AATGATCCATTTCCTAAGCAAGTTATGAATATTTTACAACATTTTTAAAAAACTCATAGT GAAGTGTGTTCTGTTGTAAAGATAAAATAATGGATACATTGTTGTAAATGGTAAAATATT AATTTAATTTTAATTATTTTCTTAGTTCTTCTTTTAGTGTTGATATATGTTTGTATTATG AGATATATTTTCTGTACTATTTGTGCAGTAAATGGTAAACACTCATAAATGTATCTATTG TATTTTATTAATCAGTCCTAATGAGTTCACTAATGTGCAGCAAGTGCTAAACAAACAGCA CCTTTTATGCAATTGTGTCAATTTTGCTGTTATTGTTCCCCATATCTGCTCTGTCTAGTG GAGGATAGACAGTGCCGCTACCTCCACCTATGAGATAGGAAATCCTCCAGACTACCGTGG TCAAGCCTGCATGAAGAGGCATAATGTGCCCATGAGGCACGTGGCCAGGCAGGTACGACC GCACACACTTCTTAGCACTAGCTAG
\end{abstract}

\begin{abstract}
ALD15D21
GTTGGTACCCGGGAATTCGGCCATTACGGCCGGGCAACTGGATTTCATAGTCCGCCATTT TTTCCCCCGCTGTGTGATTTTGTAGTCCATAGCGTCGTGCAGACTATGCAAGTTCAGAAG TCAAAATGTTTGGTTCTGGGTGTGTAAACCCACATTATTATCTACATAGACTACCAGAGT CAGAGCAGAGCTTCTTCAAAGTACCTGGTTAAGTTTCATTTTTCAAGGAAATGGACCCAC AGCAGGATGGAAAGTGCTTGCAGACAACAGAGGAGGAATGTCACAAAAACGGTGAGTTAT
\end{abstract}


GAACTGTAAATGTGTTTTAACTGTCTAATTTAGCTGTTTCTACCCTCTGTGTCGGAATAT GAGGGGAGAAAAACAGCTGTGTTTAAGTTTGTTTACCAAATGGCTGCGCAGCACCGTGTT ACTTTCATTTCGCCCCACGCTGTAATCAGCGTGTCTGCTTGTTTTTAAATGCTGGAGCGT TTCAAACTGTGATCTAAACTTAACGTCAGCTGTTTTAAATGTATTTTTTGCCTGGTGTTA AAAACTGGAGCAGCAGACTGAGGCAGAGAAGCTGTGAGCGCTGTGTGTGATAAGTCCTCT TAAGGATAGCAAGATGGATGTGTGCCTCTCCTTTTTTTCTCTGTGTTGCTGTTTTTGCAT CAAAAAAGCTGGGAACATAGTCATTTTAAGTAACAAAATATATTAATCTT

\begin{abstract}
ALD04D03
GTTGGTACCCGGGAATTCGGCCATTACGGCCGGGGGAATTATTAAGGAGATTTACGGAGT AGCGAAGCGTTCACTTTTTCTCGTCGGGAACGAAAAACCACAGCCACACCTGGGAAACCG ACAAGATAGCATGCTAGTAGAAGTGACACCACTTCAGATGAGGGAACATGTTGGCTATGG ACCAAGGAGTGGTGGAGGAATGGCTGTCAGAATTTAAGACCCTCCCTGATAGTGCTGTGG CCACATATGCTGCATCGCTTAAAGACAAAGTTTCTCTGGTCCCTGCTCTCTATAAGGTCA TCCGGGAAAACTACAGCGATCTACTGGAGCCCGTGTGTCACCAGTTGTTTGAGTTTTACC GGAGCGGTGTGCCACAGCTGCAGCGTTTCACGCTACAGTTCCTGCCAGAGCTCCTGTGGA GTCTCCTGTCCGTCAGCGCAGCCAGAGACCCCCACACTTCTGGCTGCATCGAAGCACTGC TGCTGGGCATTTACAACCTGGAAATAGTTGATAAAGATGGACAAAGTAAAGTGTTGTCTT TTACAGTTCCCTCCATATCCAAACCCTCAGTGTACCACGAGCCTTCAGCTATTGGCTCCA TGGCTCTTACAGAAGGAGCTTTATCCCATCACGGTCTGAGCAGGGTCGTGTACAGCGGGC $\mathrm{CA}$
\end{abstract}

\begin{abstract}
ALD37P02
TGTTGGTACCCGGGAATTCGGCCATTACGGCCGGGTGGAAGTCATCACTGTGGCATCAAT GCAGACTCCATGTCGCTGCACTAATTCTTATGCTTACTTATAAAAGGGCTTATGTGTCAA GGCACACTTTTAATATATACTCATTTGTATCGTCCAATTGTTTTTCTTTTTTAGTTACAC ACACAAATGAGTACTTCTGTTTTGGTGGTGAGTTAAATTAGAAGGTGTGTGGAGTCTGCG TGAGTGGTATATTTTATACTTTTTCATGGAACTGATGGATGAATAAACGTTTGAAGATGT TTTTTGTAATCATATCTCTATATATTCAGTCTATTCTTCTAGTCTAACAAAATACAGTCA TATAATCAACAAGTCTCTAGGTTTATTATAATTAAAGGATAATTTTGTTTTGCACAATTT CACAGCAGTGTTTTCATATCCAGTGAAAATGTTTAAATCATTTAAAAATTCTGCAGTGTT TATAAGAACGGGTCACCTGAAACTATAATCTTTACTCTGTTTATTTTTTTTAATGTTAAT AAATTTTAGTATCAGTTATGGGATTTAACCTTTAGGAATAGACGTAACTTTGATACATTT ATGCAGCTGGCTTAAAAAAAAGAGCGAACAATTTTTTATAAAATTTTGTTTCGCCATTAA GAATTAAAT
\end{abstract}

\begin{abstract}
ALD37P01
TGTTGGTACCCGGGAATTCGGCCATTACGGCCGGGACTCATTCAGGGTTACGACAACTCT GAGAGCAGTGTGAGGAAGGCGTGTGTGTTCTGCCTGGTGGCCATCTACGCAGTGATAGGA GAGGACCTTAAACCTCACCTCAGCCAACTCTCCAGTAGCAAGCTGAAGCTGTTGAATCTG TACATCAAGCGGGCCCAGTCTGGCTCCAGCGGCAGTGACCCCTCAGCTGAAGGCCTATAG AAGTCACACAGTTCTTCCCCCCGGGAACCACCACAGAACTGCAACAAATCAAAAACACCC GCTCACGACATCACACCCCAAACATAGTCCAAGACTCACAGAAGAACTCCGCTGACGTTC ACAGCAAACATGATGCACACACACACCTTCACCCATCATTTTTTACTTCCCCCTCTGTTG CCATCCTCGCTCATGGTTTCTTCTGTTTTCTCTTCTCCGCTGAAAGGTTTGTAGAAATGT ATTATTTACAGCTCTGAGAAAAATGTCATGTTCTCATATTTCTCTTTTCCTGTTCATTGT CTTTTTTTCCTCCTTCCTCTTGTAACATTCTAGTTTTCTCTGTGCATTTACAAAGCTGTG
\end{abstract}


CTTACAATAAAATGAACTTGTACAGAT

\begin{abstract}
ALD37G10
GAATTCGGCCATTACGGCCGGGGGTTATTGCAAATACGTGAAGTTTACCGCGCCGTTTCA AGAGAACAAAATGGGCAAAAAACAGAACCCCAAAGGCAAGAAAGACGGCCAGACACAAGA GGAACCTGAAGAATTTGTTGTTGAAAAAGTGCTGGATCAGCGGCTGGTCAATGGAAAAGT GGAGTTCTTCCTGAAATGGAAAGGATTTACAGAGGCTGACAACACCTGGGAGCCTGAGGA GAACCTAGACTGCCCTGAATTGATTCAAGCCTTTCTGGATTCGCAGAAGGACATCAAGGA GAAACCTGCTGCTGTTAAGAGAAAGGCATCAACAGACGGGACATCGTCAGAACCCAAGAA GCAAGATGTGTCAGGGAAACCACGAGGCTTTGCTCGGAACCTTGACCCAGAACGAATCAT TGGTGCAACAGACAGTAGTGGGGAGTTGATGTTTCTGATGAAATGGAAAGACTCAGATGA AGCAGACTTGGTCCCAGCCCGCGAGGCCAACACTCGCTGCCCTCAGGTGGTCATATCTTT CTATGAGGAGCGACTGACGTGGCATTCCTGTCCAGAGGATGAAGCCTAGTAATTCTTGCT GTTCCTTTCACCTCCTCTGCCCTCTGGCCTGCATTTTAGCTTTAGCTAGTACCTTTAGAT GTTTTTGAAACTGTT
\end{abstract}

\begin{abstract}
ALD28L22
GTTGGTACCCGGGAATTCGGCCATTACGGCCGGGGGATAAAGAAGGTTTCATCGCTGTCG AGCGATTAGTCCTTGAAAGTGAGCTGAGTTGTATATCACTTACAATTTATCGAACAGCCT TAAATAGGTTTTTGTTGGCAATAACATTGTCAGCAAACCTTTATCGGTTTTGGGTCCATA AAGAAAATGGGAACTGATAAAAGGATCAGTCGGACCGAACGCAGTGGTAGATATGGATCT GAGCAGGCCCGTGATGAGTCGGACTGGCGAGACAGACGCGATCGGGACCAAGAGCGGGAG CACGGCAGCCGACGCTGGAGTGATGAGAGGCGTGATCGTTATGAAGGAGATCGCAGAAGC TCTCGAGACAGTCCAGAGCAGAGAGAGAGGAAAAGGAGGAACAGTGACAGATCAGAAGAT GGTTATCACTCAGATGGAGACTATGGAGACCATGATTATAGAAGGGATCCAGGGAATGAT AAGAAGAGTAAGACCATCATGCTTGGGGGTTTATCTCCTCAAACTTCTGAGGAAGATATT CGTTTCGTCATCGATCAACTAGAGGGACCCCAGCCAGCAGATGTCAGACTGATGAAAAAG AGAACAGGTATAAGCCGTGGTTTCGCCTTCGTGGACTTTTATCACTTGCAAGATGCTACC AGATGGATGGAGACCAATCAGAAGCGTCTGGTCATCCAAGGCAAACTCGTGGACATGCAC TACAGTCATCCT
\end{abstract}

\begin{abstract}
ALD35M13
GTTGGTACCCGGGAATTCGGCCATTACGGCCGGGGGGGTGTGGATCTACAACAGTCTGCT GCTGGAGACAACCACGGACTGTTTGACAAATACCTTCTCCTTTGGCTGTACTACAGTAAA AACCGCGGACATACCAGTGCCTTAGAGCGTCTCTGGTTGTGTCCCAGATGAGGGACTGAT GCTGGTCAGAACTTGCCTAAACTTGGTCCTGGTTTAGTTCTGCATCTGTTCTCACTGGTG CCCCTTTGAGAAAAGAAGAATACATCAGTCGAGATGGATGATATCTTCACACAGTGCCGG GAAGGCAACGCCGTGGCTGTTCGCTTGTGGCTGGACAATACAGAGAATGACCTGAATCAG GGAGATGACCATGGCTTCAGCCCGCTCCACTGGGCGTGTCGGGAGGGGCGGTCCAATGTG GTGGACATGCTCATCATGAGAGGAGCACGCATCAACGTCATGAACCGGGGGGATGACACT CCTCTGCACCTGGCTGCGAGCCATGGACATCGGGATATTGTGGGGAAGATGATCCAGTGC AAAGCAGACACCAATGCAGCCAACGAACATGGAAACACACCACTGCATTATGCCTGCTTC TGGGGCCATGACCAAGTCGCTGAGGACCTCGTGAGTAGTGGGGCTCAGGTGAGCATCAGT AGCAAATTTGGCGAAACTCCCTTGGATAAAGCCAAACCTCATCTGCGTGACCTCCT
\end{abstract}




\begin{abstract}
ALD37M10
GTTGGTACCCGGGAATTCGGCCATTACGGCCGGGCCCACATCTTACAGACTTCGTGATTG CTTTCTGTTTAATGTCTGCCCATCAATAAATGACTTTATAATCACACCCGCAAGACTTGT CTTTTAAACACCCTTTTCGCCGTGGTTCTGACCATTCTTGCCGCGACACAAATGTAAACG CTGGCTTTGGAGGGCGGAGTTAATGTCAACAGATTTTTGGAGGGCGTTACCAACAGAGCT CATTAACATAAACAGCTCTTCTGAACGGAAGTCAGAGCAGGCAGAACTAGAAAATGGGAA ATTTTATTATCTGAGAATTATTTTGTGCATTAAATTGAATGAACATGGTCTGGATAGTCC ACAGACATACCATAAAAATTTCTAGGACCCATAATTGGTCTCCTTTAAAGATTACGTCGC ATCAACAGGAAAAGAAACAAGCACGACATCCGTATTTTCAAAATAAGACACTATTGGGTG GAATAATTTGTCGGAATAATGTCGAAATTGCAGCTACAGTATATTAATAAATATACGGAT ATATTTCTACACAAATGCTGTCAGAATGTATCTTTCTGTTTGGGAAGCAGATAGAAACAT GTAGCATGAAGCTATGTGTACAAACATGATACGCATGGATTTTATTTTGAAATTACAAGT TTCAGAAAGACGACAGTGAGCAGTGTTGCCAGGTGTACAATAATTATTGCATTTGTACCA
\end{abstract}

\begin{abstract}
ALD35H01
GTTGGTACCCGGGAATTCGGCCATTACGGCCGGGGGCAGGAGGAAAGATGGCTTCGTCCA TGAGTGGGATGTTTCCCGGTCAGCAGCCGCCTGGTTCGCATCCTGTCGGTGGTCCCGGTG GACCGGGTCAGCCTGGCTTTCCTGGTACCGCTCCTCGGCCCCAGGGCAATAACACTTTGG TGGATGAACTAGAAGCTTCGTTTGAGGCCTGTTTTTCTTCATTAGTAAGTCAAGATTATG TCAACGGAACAGACCAGGAGGAGATTCGAACTGGAGTGGACCAGTGCATACAGAAGTTCC TGGACGTGGCTCGACAGACCGAGTGCTTCTTCTTGCAGAAGAGACTTCAGCTGTCTGTGC AGAAACCAGAGCAGGTGGTGAAAGAGGATGTGTCAGAGTGGCGCAACGAGCTTCAAAGGA AAGAAATGCTGGTTCTGAAGCATTTGACCAAGCTGCACCACTGGCAACAAGTACTTGAAA ATTTGAGTGGTCACCATCATAAACCCACAGATTTTCCTCCTCCCGGACCTCTGGCCTTTC TGG
\end{abstract}

\begin{abstract}
ALD37M09
TGTGTTGGTACCCGGGAATTCGGCCATTACGGCCGGGGCAGCGAGTCGTAAACTCAGACT TTATTTTTTGTTAGTTTTGTTTTTAGAGGCGATAAAATCGAATCTATTTTTGCTGTTTTC ATCAGTTTTTTCTTTCCTTCACTCGTTTTTATTTTCACAGCTGCTCGTTTCTCTGGAACC GTATAAGAAGGAAACAGTCGTCAGTTTTCTAAGTTTAACTCCAGAGCAGCTCTGACCTGA TAAGACCTTCATCCTGTTTTTGGCCTCAGTTTGACGTTTCTGTGATCGTCTCTGCAGCCT GACAGGAACGAGGAAGTTGTTCAGTTTCCTGTTGAAGGCGTCTGACTGGAGATGACTGGA AACTGGGGGAAAAAATCTTAGTTTTAGTTCAGTTTAAGCTGCAGTGGAGTCAGATGTTTG TCTCTGGTGACCTCTGGCCTCTCTGTGTTTCCAGTTTGCATCAGTTTCAAAATAAAAGCG TTAAACTGCAGGTCTCATCGTTGCCGGCGGCCTGCAGTAACCGTGCAGCAGAGCGAGCTC GTGTCCTGCTGGAGGTTTGACGGCGATGCAACACGTCCTCGTCCTCGACAGCTCCTCCAC AGCGAGCGCCATCTGTCACATCGCCTCATCTTCCAGGAAATTACTGCTCGGACCGAAAGC CTCAACATTATCTGATACGTACCCCGGCCGGGCCCGAATGAACTC
\end{abstract}

\begin{abstract}
ALD40K12
GTTGGTACCCGGGAATTCGGCCATTACGGCCGGGGTGGTGACTGCTGGTGCCCGCCAGTA GGAGGGCGAGAGCCGACTCAACTTGGTGCAGCGCAACGTTAACATCTTCAAGTTCATCAT CCCCAACATCGTCAAATACAGCCCCAACTGCATCCTGCTGGTGGTCTCTAACCCAGTGGA CATCCTGACCTACGTGGCGTGGAAGCTGAGCGGCTTCCCCCGTCACCGTGTCATCGGCTC AGGCACCAACCTGGACAGCGCCCGTTTCCGCCACCTCATGGGGGAGAAGTTCCACATCCA
\end{abstract}


CCCTTCAAGCTGCCACGGCTGGATCATCGGAGAGCACGGAGACTCCAGTGTGGCTGTGTG GAGCGGCGTGAATATCGCTGGTGTTTGTCTTCAAAACCTCAACCCACAGATGGGAGCTGA TGATGACAGTGAGAACTGGAAGGATGTACACAAGCAGGTGGTCGATGGGGCCTACGAGGT CATCAAGCTGAAGGGCTACACTTCCTGGGCGATCGGTATGTCTGTGGCTGACCTTGTGGA GAGCATTATGAAGAACTTGCACAAAGTTCACCCCGTCTCTACACTTGTCCAGGGCATGCA TGGTGTGAAAGACGAGGTCTTCCTGAGCATCCCTTGCATTCTGGGCAACAGTGGCCTGAC GGATGTCGTCCACATGACCCTGAAAGCTGAAGAGGAGAAGCAGCTGGTGAAGAGTGCCGA AACCCTGTGGGGCGTACAGAAGGAGCTCACCCTGTGAA

\begin{abstract}
ALD40D17
GTTGGTACCCGGGAATTCGGCCATTACGGCCGGGGCACTTTGTTGATTGGGTTTTAGCGA GCTGTACTGGAGCCAATCAGAGGGAAACCGGACAGTGAAGCTACGAGGTCAGAAAATAAA AAATTAAAGGCATGGAGCAGTACACCACTGCCACCACCACCACAGGAGAGCAGATCGTTG TGCAGACTGCCAATGGACAGATCCAACAGCAGGTCCAGGGACAGCCCCTGATGGTACAGG TGAGCGGTGGGCAGCTCATCACATCCTCAGGGCAGCCCATCATGGTGCAGGCCATGTCGG GGGGTCAGGGTCAGACCATAATGCAGGTTCCTGTGTCTGGAGCTCAAGGACTACAGCAGA TCCAGCTGGTGCAGCCGGGTCAGATCCAGCTGCAAGGAGGGCAGACTCTCCAGCTCCAGG GTCAGCAGGGTCAGCCTCAGCAAATCATCATCCAGCAGCCTCAGACCGCCATCACTGCTG GACAGAACCAGGGACAGCAGATCAGCGTTCAGGGCCAACAGGTCGCTCAGACGGCAGATG GACAAACTATCGTCTACCAGCCTGTGAACGCAGATGGCTCTGTCCTGCAACAGGGAATGA TCACGATTCCTGCTGCCAGCTTGGCAGGTGCTCAGATCGTACAGGCAGGGAACGCCAGCA CCAACACCAGCAGCAGCGGCCAAGGCACTGTGACCGTCACCCTGCCTGTCTCCGGCAACA TGGTCAATG
\end{abstract}

\begin{abstract}
ALD41M14
GTTGGTACCCGGGAATTCGGCCATTACGGCCGGGGATAGTGGTATTTTCGTGCTCTTGGC GAAAATACTCATCTATTCATAGCCGTCAGCTAGCTTTTTTCCCCTTTATTTTTCTTTTTT TTTTAACCGGTTCGTTCGCTGGAGGTAGGCAGGCTTATCTCGGCAGTGTATATAAACTTA GTAGCGCATTATCATGGAGCTCTGGATGTTAAGGAACATCTCCGAACGAGAAGCCGAGCA GCAGCCGGTTTCTGCTTCTAGCAGCTAACATTAGCTTTCATTTAGCACTTCCCCGGCTTT TTTCTTCTTTTTTTTTTTAAACTCGACGACTTGGCTCGGGTAGCTTTCTGTGACTGCTG GGTTATATATATTTTTTGTCCAGTTAGCAGCCCTGCGGTGCCCCGACTTATTTATCATTT TGATATCTTCGACATGTCTGGGGTCTAAGTTTACCTGTGAAGTTTTTAAACCAGGTGTTT TTGTTTTAATCGAACATGAAGTCCCCACTGGCTCCCGGTATTCGACTAATAACGAGTTTT TCTCGGGATAGTTGGTATCGTGGTTTCATCCTCTTCCTCACGTTCCTCTTCTACACAGCC TACCATCTGTCACGGAAACCCATCAGCATTGTTAAGAGTGAGCTGCACAGAAATTGTTCA ACGGTTATCCGGCCACCAGATTTGAACGTAACCAACAATGAAACCTGGTGTGACTGGGAG CCCTT
\end{abstract}

\title{
ALD23L08
}

GTTGGTACCCGGGAATTCGGCCATTACGGCCGGGAAGGATCCATCGGACCTGTCCTTCGA TTCGGGGAAAAAGAAGAACGTATTTGTCGGACGCATCGGAGGAGTCTTCCGTTTTGGACG TCCTTCGTCGCGGCGCTGTGACGTATGCGGCCGACAAATTCGACCTTCGGAGGACCCGTC CTGCCGTTTTGGACACAGCCGAAGTGTATTGTAGCATACCGACAATGTCTACGTGTATTG TAGCATACCGACAATGTCTACATGTATCGTAGCCATCGACGTGTCCTACGTGTATCGTAG CTACCGACGTGCCTACGTGTATCGTAGCTACCGGCGTGTCCTATGTGTATCGTAGCTACC CACGTGTCCTACGTGTATCGTAGCCATCGACGTGTCATACGTGTATCGTGGCTACCGACG 
TGTCTACGTGTATCGTGGCTACCGACGTGTCTACGTGTATCGTGGCCGTCTACGTGTATC GTGGCCGTCGACGTGTCTACGTGTATCGTGGCCATCGACGTGTCTACGTGTATCGTAGCA CACCGACGTGTCTACGTGTATCGTAGCACACCAACGTGTCTACGTGTATCGTAGCACACC GACGTGTCTACGTGTATC

\begin{abstract}
ALD41J01
GTTGGTACCCGGGAATTCGGCCATTACGGCCGGGACAACTTTTGTTTGCAAAGATTTGCA CAAAGTAGTGGGAAATGATGCTTCGAGCGGCTCGAACCAAAACTACGATGTTAACATCTG AGTGAAACTCTTCTCCTGTTTAAATGTGAGAAAGATGACAAATCGTTTAGTTTTGTTGCC AATATTATTTTTTAAATTTACGAAACATCTTGATGTAATTCACAGTGATTGAATGTTTTA GCTGAAGTACTTTTTCTTTCTTTCTTCTTGTTAATAAGTTTTATTTAACAGAAACATTAC AAATATGTGACTCATCACATCGAAACAAGGATTAAGTCCAAAAAGTGAAATGTGGAGAAA TGGGCAGATATAGGGTTTTGATTTAAACGTGTGCAAAAATTGCATTTTGTCAAAAAAAGA ATGAATTAATGCCTTATCAATGTATCATTTGAATGAACTGAAATATAATCCTAAAATTTT TTTACAGCATTTTTTGAGCAGTAACAACAAAAACAGATTGAGGAAAAACACCTTCAAATT TTGAATTTTTCCTTGTGAGCTAGAAATGTTTTTACTTCTTTCCAACTGGGAAATCTTAAT ATACAAATACTATTTGAGGTTTCCTCTTAATATTAAAATAAAAAATGACTCAATAAATTT ACATAAAATCAGGAATCAAACCAAACAGTAATGTATCATTGTAACCAACAAGACAAATTA AGTCATTTCTCTGGATAAGCTGATGATATCAGGATGATCCAGGTATCAA
\end{abstract}

\begin{abstract}
ALD35M04
GTTGGTACCCGGGAATTCGGCCATTACGGCCGGGGGTTGACAGTCGTGGTATAAGTGTGG AGGCTAAGCTACATGTGGAGGGTGAAACGGGAGGCATCCCCGACCCTAACTTTGTAAAAC AAATATCACCTTTTACTCGAAACAACGCTAAATTAAGTCTAATTGTAAATAAGGATAATA TCTTTTTTAAAATGAAAGGGTGAGTATGAGCTTTCTGAGTGACGGACGTGTAGCTTAGCA TGAGTAAAGTTTTTCAGGTCAAGGGAGGCGGGCAGGAGACGACGAGGTTAGCTAGCGCGC CGGATCGAGCTGGGGGACGTTACGCCTCACAACATTAAACAGCTCAAACGCCTGAACCAA GTCATCTTCCCTGTCAGCTACAACGACAAATTTTACAAAGATGTACTGGAAGTGGGGGAG CTTGCGAAGCTAGCGTACTTTAATGACATTGCAGTGGGAGCTGTGTGCTGCAGAGTAGAT CACTCTCAGAACCAGAAGAGGCTGTACATCATGACTCTTGGTTGTCTAGCGCCCTATCGT AGACTTGGAATCGGCACAAAGATGCTTAATCATGTCCTGAACATCTGTGAGAAGGACGGG ACTTTTGACAACATTTACCTTCATGTGCAGATCAGCAACGAGTCAGCCATCGACTTTTAC CAGAAGTTTGGCTTCGAGATCATCGAGACAAAAAAGAATTACTACAAGAGGATAGAGCCT GCAGACGCCCACGTGCT
\end{abstract}

\begin{abstract}
ALD37K15
GTTGGTACCCGGGAATTCGGCCATTACGGCCGGGCGTCAGAGGCTCATCCACCCCTTCAG AACCCACAGCCCAGCTCCCACCTTCCCTCAGTGCTATCCCCTGCGCTCTCACACAACGTT TCACACGCCAACAACACTGTTCCCCACTTCTCAGCCCCCGCACCTCGCGTCTCCCATCGT ATGCAGCCACCAGGGCCTTACTTTCCCCTTTCGGAGCAGCAACAACAGACACCGCAGCAG CAGCAGTCTGTTTTTGTGCCCTTCAGCGCTCAGCAGGAACCCTCAAAACAGACCCAAAAC CAGACATCCCAGCCAACGAATCTGCCCCCAACAACCCAAGCTCAGGCCCAAGCTAATCTG GGAATAATGAACGGCTCCCAGATGCAGCATGTTGCCACCGCCGGCAAACCTCAGCAGATA CCCCCCAACTTTGGTCATGCAGGTCTCTTCAATTTCAGCAGCATCTTTGATAACAACAGC CAGGTGGGAAACAATCAGGTGTGGGGTGCATGCCATTTGCCTGCACGATCTCCTCCAGAT CAGTCATACTCTGCCCCACCAGCCTATATGAACATGGGACAGATGGAGAATATGATGCCA CCTCCTTCAGACAACTCCAAAGCCCCCGGCTACCGTTCTAACTCTCAGAGGATCGTCAAC
\end{abstract}


AGCCCCATTGCTTTGACCAGCTATG

\begin{abstract}
ALD28D17
GTTGGTACCCGGGAATTCGGCCATTACGGCCGGGGGTTTTATTTGTATTTTTTTCCTTTT TTTTATTTATTTTGTTGTAGAAATTGTTTCATGTTTTTAACCCTCAGCTGATGCATTTTG CTTTGTTTGCCGTCCTGCCACCTCGTTCATGATGCGCCAAAATGCTCTGAAGGTGAAAGG GTAGATTTGGGTTGAGATTTATCAAAAAAAAAACTCGTGCTATGTTCACCTACAGATGCC AAAATATTTTTTTTCCTGTAATAAATATGAAGTGACACTTCTCATGGAGCAGCTATATAC CATGAAGGACTCTACGGTTTTTGCTGAGACTGATGAAACTTATTAATCCCAACGTAGATT AAATTTAAACTAATATATGAGCATTGTTTGAAGTAATGTCTTGTTGGTATGATATTTGTT TTTGAAGGAATCTGTGCACAAATCTTTATTTCTGGGAGAAGCGCCCATAAAACCTCATAT GAACACCAGATTTAAGAAACACACTATTCGTTATTTTAGCCCCAAGATCTTTTATTTCAA AAGGAATGACAGCTTTGGTTTGAATCTAAATAAACTTTAGATGTAGGTTAATCTGTCCGT TCGATATTGAGCAGTTTATTTTAATTTTAAAGTTGTTTTGGGATAAAACGAATCTTGTAT $\mathrm{AT}$
\end{abstract}

\begin{abstract}
ALD18J24
GTTGGTACCCGGGAATTCGGCCATTACGGCCGGGGGGACAGAGACAAAAACAGGAGACAA GAGAGGAGCTCAGAGGACAAGGACAGTGTTCGTGTCTGTTCCGCTCAGGTCCAGGTCCAG GTCCCCGGGGCTCGCTCATCGTGCAGGAGGGTGAGGACACCTGCAGCGGCACCGAGCCAT GGTGAAGATCAGCTTCCAGCCTGTCGCAGGGCAGAAGGTGGAGAAGGAGAGCGATGGCGA CAAGACAGAGATCCTGATTCCTCATCCGCTGGATGAAGATGAGCTGGTTCTGCCACTGCG CCCCAAGAAGTCGCCACTTAATGGGCTTTGCTGCCTGACGTTTGGACTGGTGGTCTTTAT GGCTGGACTGGTTCTGGCCTCCATCTACGTCTACCGCTACTACTTCATACCTCACATCCC AGAGGAGAACCTGTTTCACTGCAGGGTTCGATATGACGACGCGGCATACGCCTCACTCCG TGGCCACCAGGAACTGGAGGAGAACGTTGGCATCTACCTCGCTGACAACTACGAGAAGAT CAGTGTCCCTGTCCCCCACTTCAGAGGCAGCGACCCCGCTGATATCATTCATGATTTCCA TAAGGGACTTACCGCCTACCATGACATTGCTCTGGACAAGTGCTACGTCATTGAGCTCAA TACCACCATTGTGATGCCTCCACGCAACTTTTGGGAGCTTCTCATCAATGTTAAGAAGGG AACATACCTGCCTCA
\end{abstract}

\begin{abstract}
ALD03J18
TGTTGGGTACCCGGGAATTCGGCCATTACGGCCGGTGGTTCCAGCTCTCAGGGAGGCGTG TTCTCTAACAATCGTAGGATGGGAGACAGTCGGAGCGGCATGATGGCTTCTTCTAGTCAC TCGTCAGGACTAAGCCGGATTGTACAGATCACGAGCAATTCAATGCCCTCCAGCGGCAGC ATGAGTGGCTTCAAACCCTTCAAAGGAACACCTCGGCCGTTCTAAAAGCAACTGTTTTTT TтTтTтTтTтTтTCCATTTTTGAAGCATCAAAAATTTGAAGGCTTTTTTTTTCCTAAAA TTTTGTTTGTAATGTTTAAAATGTCAACAGTTTTTGGTGGTTCATTTGCAACTTTTGAGG ATAACACTTTTTTATGTATGAAATATTTACCTCAGTGTAGAGTTTTCCTTGCAGTTCTGC TGTTACCATAAACAGCCCCGTTAACGCCTCTTATTTAGAGCAACATTGTACAGCCCTCAG TCTTTGTAACGTTTTCAAGATAATTCTCCTGTTGAGTGATATGATTTCAAAAGTGTAGCG TTACACTTTGTAATGTCTTATTTTAGTGTATTATAGAATTCAAAATGACCAAGATATTGA CTTGGATGTGTCACTGTTTGAAATTGATGACATTTTTTTTCTAGTTTTGTTTTTTAATTT AGTTTTTAAGTCATACCTG
\end{abstract}

\title{
ALD14P11
}


TTGTGTTGGTACCCGGGAATTCGGCCATTACGGCCGGGGGATAAAACCACTTCTCGCAGC ACAAACCTCGCTTCAAAGGCGGTAGTTAGCTTATTTGTGACAAAACATCGCCTTTTTGGG TGTTTTATCTCTTGAACAAGGGGTCAAAGTCGCTAAAGTATCGATTTCCCCTCAGTTTTT CCCAGTTTCATCGCTGTAAGTTAAGTTTAACAGCCGCCGGACAACGTAAAGCTGGGGAAG TAGAGCTGGAATAAAAAGCGGACCTTTTCAACAGAAACACCTGCCAACGAGCCCTCACAC AGGGGCGCAGGAAGCCCAGCAGCCTTTGGCGTGGAGAGTGGGGAAGTGAGCAGAGGAGGT CGAGGCAGCAGCCCAGCCATGGGGAAGCAGAACAGCAAGCTGCGGCCGGAGGTCCTGAAC GACCTGCGGGAGAACACGGAGTTCTCCGACCACGAGCTGCAGGAGTGGTACCGCGGCTTC CTGAAGGACTGCCCCAGCGGCCACCTGACCGTGGAGGAGTTCAAGAAGATCTACGCCAAC TTCTTCCCCTACGGCGACGCCTCCAAGTTCGCCGAGCACGCCTTCCGCACGTTCGACACG AACGGCGACGCCACCATCGACTTCAGGGAGTTCATCATAGCGCTGAGCGTGACGTCGCGC GGCGGGCTGGAGCAGAAGCTGCGCTGGGCCTTCAGCATGTACGACCTGGACGGGAACGGT TACATC

\begin{abstract}
ALD09P18
GTTGGTACCCGGGAATTCGGCCATTACGGCCGGGGGCATTGCGGTTTATCATGGCGACTT CAACGATTTGACCCTTGCTAGCCTAACTCAAAGGACCAGCTGAAGATAAGCAAAGCCTGT GCGGAAAGTTTGAGTCACTTCACTTTATACTTGAGGATGAAAGTGTTGTTTACAGCGCTA AAACGAACCTATAAGGCTGCTGGTTACGTCTGCCGCCGGAGTTCCCACGCTGGCAGCGGC CCGGTGTTGCCTCTCGCAGGTGAGGTGATAGTGGGGTCCAGCCGGGAGCTCGTGCGAGGT CTAGGCTCTCAGGTGGAGGTGAGGACGAGCTTCATCACTGAGGAGGAGGAAGCTGCTTTT CTGCACGAGCTGGAACCAGGACTGAAGAAGAAACGCTACGAGTTCGACCACTGGGACGAT GCAATTCATGGCTACAGAGAGACCGAGCGGCTGAGGTGGGGCCCAACCTGCGAAGAAATC CTGAACCGTGTCCGGTCTGTGGCATTTCTTGACAGCAGTCCTCTCCTTGGCCCCGTGCAT GTTCTAGATTTGGACAAAACCGGCTACATTAAGCCACATATCGACAGTGTGAAGTTTTGT GGCAGCACCATTGCTGGCTTGAGTCTTCTGTCAGACAGTGTTATGCATTTAGTAAAGGAG GACGCTCCGCACGAATGGGTGGACCTATTGCTCTCTCGACGCTCTCTCTACATAATGAGG GACCAGGCCAGATATAACTTCACTCATGAGATCCTCGAAGATGAGCAGTCTGTGTTTAA
\end{abstract}

